\title{
Theoretical description of the neutron beta decay in the standard model at the level of $10^{-5}$
}

\author{
A. N. Ivanov $\oplus^{1,{ }^{*}}$ R. Höllwieser®, ${ }^{1,2, \dagger}$ N. I. Troitskaya, ${ }^{1, »}$ M. Wellenzohn, ${ }^{1,3,}$ and Ya. A. Berdnikov ${ }^{4, \|}$ \\ ${ }^{1}$ Atominstitut, Technische Universität Wien, Stadionallee 2, A-1020 Wien, Austria \\ ${ }^{2}$ Department of Physics, Bergische Universität Wuppertal, Gaußstraße 20, D-42119 Wuppertal, Germany \\ ${ }^{3}$ FH Campus Wien, University of Applied Sciences, Favoritenstraße 226, 1100 Wien, Austria \\ ${ }^{4}$ Peter the Great St. Petersburg Polytechnic University, \\ Polytechnicheskaya 29, 195251, Russian Federation
}

(Received 14 May 2021; accepted 22 July 2021; published 30 August 2021)

\begin{abstract}
In the framework of the Standard Model (SM) a theoretical description of the neutron beta decay is given at the level of $10^{-5}$. The neutron lifetime and correlation coefficients of the neutron beta decay for a polarized neutron, a polarized electron, and an unpolarized proton are calculated at the account for (i) the radiative corrections (RC) of order $O\left(\alpha E_{e} / m_{N}\right) \sim 10^{-5}$, i.e., $O\left(\alpha E_{e} / m_{N}\right)$ RC, to Sirlin's outer and inner $O(\alpha / \pi) \mathrm{RC}$, where $\alpha$ and $E_{e}$ are the fine-structure constant and the electron energy, respectively; (ii) the outer $O\left(\alpha E_{e} / m_{N}\right) \mathrm{RC}$, caused by Sirlin's outer $O(\alpha / \pi) \mathrm{RC}$ and the phase-volume of the neutron beta decay, calculated to next-to-leading order in the large nucleon mass $m_{N}$ expansion; (iii) the corrections of order $O\left(E_{e}^{2} / m_{N}^{2}\right) \sim 10^{-5}$, caused by weak magnetism and proton recoil; and (iv) Wilkinson's corrections of order $10^{-5}$ [Wilkinson, Nucl. Phys. A377, 474 (1982)]. These corrections define the SM background of the theoretical description of the neutron beta decay at the level of $10^{-5}$, which is required by experimental searches of interactions beyond the SM with experimental uncertainties of a few parts of $10^{-5}$.
\end{abstract}

DOI: $10.1103 /$ PhysRevD.104.033006

\section{INTRODUCTION}

A contemporary level of sensitivity of about $10^{-4}$ or even better for experimental investigations of the neutron beta decay [1-3] with a polarized neutron and unpolarized electron and proton [4-7] and with a polarized neutron, a polarized electron, and an unpolarized proton [8] demands the theoretical description of the neutron beta decay within the Standard Model (SM) $[9,10]$ at the level of $10^{-5}$. As has been shown in [11-14] Wilkinson's corrections [15] provide the SM contributions to the neutron lifetime and correlation coefficients of the neutron beta decay of order $10^{-5}$. Of course, they do not exhaust a complete set of the SM corrections of order $10^{-5}$.

\footnotetext{
*ivanov@kph.tuwien.ac.at

roman.hoellwieser@gmail.com

‡natroitskaya@yandex.ru

\$max.wellenzohn@gmail.com

berdnikov@spbstu.ru
}

Published by the American Physical Society under the terms of the Creative Commons Attribution 4.0 International license. Further distribution of this work must maintain attribution to the author(s) and the published article's title, journal citation, and DOI. Funded by SCOAP ${ }^{3}$.
In Refs. [16,17] we have calculated radiative corrections (RC) of order $O\left(\alpha E_{e} / m_{N}\right) \sim 10^{-5}$, i.e., $O\left(\alpha E_{e} / m_{N}\right) \mathrm{RC}$, where $\alpha, E_{e}$, and $m_{N}$ are the fine-structure constant [10], the electron energy, and the nucleon mass, respectively, to Sirlin's outer and inner (see [18]) $O(\alpha / \pi)$ RC $[19,20]$ (see also [21-23]), which are independent of the hadronic structure of the neutron (see [16]) and induced by the hadronic structure of the neutron (see [17]), respectively. In turn, in [24] we have calculated a complete set of corrections of order $O\left(E_{e}^{2} / m_{N}^{2}\right) \sim 10^{-5}$, caused by weak magnetism and proton recoil. Together with Wilkinson's corrections [15] (see also [11-14]) the corrections, calculated in $[16,17,24]$, define the SM background of the theoretical description of the neutron beta decay at the level of $10^{-5}$. In this work we supplement this SM theoretical background of the neutron beta decay by the outer $O\left(\alpha E_{e} / m_{N}\right) \sim 10^{-5} \mathrm{RC}$, caused by Sirlin's outer $O(\alpha / \pi) \mathrm{RC}$ and the phase-volume of the neutron beta decay, calculated to next-to-leading order (NLO) in the large nucleon mass $m_{N}$ expansion (see the Appendix D).

For the electron-energy and angular distribution of the neutron beta decay for a polarized neutron, a polarized electron, and an unpolarized proton we use the most general form, proposed by Jackson et al. [25-27] and Ebel and Feldman [28]: 


$$
\begin{aligned}
\frac{d^{5} \lambda_{n}\left(E_{e}, \vec{k}_{e}, \vec{k}_{\bar{\nu}}, \vec{\xi}_{n}, \vec{\xi}_{e}\right)}{d E_{e} d \Omega_{e} d \Omega_{\bar{\nu}}}= & \left(1+3 g_{A}^{2}\right) \frac{\left|G_{V}\right|^{2}}{16 \pi^{5}}\left(E_{0}-E_{e}\right)^{2} \sqrt{E_{e}^{2}-m_{e}^{2}} E_{e} F\left(E_{e}, Z=1\right) \zeta\left(E_{e}\right)\left\{1+b\left(E_{e}\right) \frac{m_{e}}{E_{e}}\right. \\
& +a\left(E_{e}\right) \frac{\vec{k}_{e} \cdot \vec{k}_{\bar{\nu}}}{E_{e} E_{\bar{\nu}}}+A\left(E_{e}\right) \frac{\vec{\xi}_{n} \cdot \vec{k}_{e}}{E_{e}}+B\left(E_{e}\right) \frac{\vec{\xi}_{n} \cdot \vec{k}_{\bar{\nu}}}{E_{\bar{\nu}}}+K_{n}\left(E_{e}\right) \frac{\left(\vec{\xi}_{n} \cdot \vec{k}_{e}\right)\left(\vec{k}_{e} \cdot \vec{k}_{\bar{\nu}}\right)}{E_{e}^{2} E_{\bar{\nu}}}+Q_{n}\left(E_{e}\right) \frac{\left(\vec{\xi}_{n} \cdot \vec{k}_{\bar{\nu}}\right)\left(\vec{k}_{e} \cdot \vec{k}_{\bar{\nu}}\right)}{E_{e} E_{\bar{\nu}}^{2}} \\
& +D\left(E_{e}\right) \frac{\vec{\xi}_{n} \cdot\left(\vec{k}_{e} \times \vec{k}_{\bar{\nu}}\right)}{E_{e} E_{\bar{\nu}}}+G\left(E_{e}\right) \frac{\vec{\xi}_{e} \cdot \vec{k}_{e}}{E_{e}}+H\left(E_{e}\right) \frac{\vec{\xi}_{e} \cdot \vec{k}_{\bar{\nu}}}{E_{\bar{\nu}}}+N\left(E_{e}\right) \vec{\xi}_{n} \cdot \vec{\xi}_{e}+Q_{e}\left(E_{e}\right) \frac{\left(\vec{\xi}_{n} \cdot \vec{k}_{e}\right)\left(\vec{k}_{e} \cdot \vec{\xi}_{e}\right)}{\left(E_{e}+m_{e}\right) E_{e}} \\
& +K_{e}\left(E_{e}\right) \frac{\left(\vec{\xi}_{e} \cdot \vec{k}_{e}\right)\left(\vec{k}_{e} \cdot \vec{k}_{\bar{\nu}}\right)}{\left(E_{e}+m_{e}\right) E_{e} E_{\bar{\nu}}}+R\left(E_{e}\right) \frac{\vec{\xi}_{n} \cdot\left(\vec{k}_{e} \times \vec{\xi}_{e}\right)}{E_{e}}+L\left(E_{e}\right) \frac{\vec{\xi}_{e} \cdot\left(\vec{k}_{e} \times \vec{k}_{\bar{\nu}}\right)}{E_{e} E_{\bar{\nu}}}+S\left(E_{e}\right) \frac{\left(\vec{\xi}_{n} \cdot \vec{\xi}_{e}\right)\left(\vec{k}_{e} \cdot \vec{k}_{\bar{\nu}}\right)}{E_{e} E_{\bar{\nu}}} \\
& +T\left(E_{e}\right) \frac{\left(\vec{\xi}_{n} \cdot \vec{k}_{\bar{\nu}}\right)\left(\vec{\xi}_{e} \cdot \vec{k}_{e}\right)}{E_{e} E_{\bar{\nu}}}+U\left(E_{e}\right) \frac{\left(\vec{\xi}_{n} \cdot \vec{k}_{e}\right)\left(\vec{\xi}_{e} \cdot \vec{k}_{\bar{\nu}}\right)}{E_{e} E_{\bar{\nu}}}+V\left(E_{e}\right) \frac{\vec{\xi}_{n} \cdot\left(\vec{\xi}_{e} \times \vec{k}_{\bar{\nu}}\right)}{E_{\bar{\nu}}} \\
& \left.+W\left(E_{e}\right) \frac{\vec{\xi}_{n} \cdot\left(\vec{k}_{e} \times \vec{k}_{\bar{\nu}}\right)\left(\vec{\xi}_{e} \cdot \vec{k}_{e}\right)}{\left(E_{e}+m_{e}\right) E_{e} E_{\bar{\nu}}}\right\},
\end{aligned}
$$

where we have used the notations in Refs. [11,24,29,30]. Then, $g_{A}$ and $G_{V}$ are the axial and vector coupling constants, respectively $[1,2,5,6,10]$; the CabibboKobayashi-Maskawa (CKM) matrix element $V_{u d}$ is included in the definition of the vector coupling constant $G_{V} ; \vec{\xi}_{n}$ and $\vec{\xi}_{e}$ are unit spin-polarization vectors of the neutron and electron [11,12,14] (see also [31]), respectively; $d \Omega_{e}$ and $d \Omega_{\bar{\nu}}$ are infinitesimal solid angles in the directions of electron $\vec{k}_{e}$ and antineutrino $\vec{k}_{\bar{\nu}}$ 3-momenta, respectively; $E_{0}=\left(m_{n}^{2}-m_{p}^{2}+m_{e}^{2}\right) / 2 m_{n}=1.2926 \mathrm{MeV}$ is the end-point energy of the electron-energy spectrum $[1,2] ; F\left(E_{e}, Z=1\right)$ is the relativistic Fermi function, describing the electron-proton final-state Coulomb interaction, and is equal to [32] (see also [15] and a discussion in [12])

$$
\begin{aligned}
F\left(E_{e}, Z=1\right)= & \left(1+\frac{1}{2} \gamma\right) \frac{4\left(2 r_{p} m_{e} \beta\right)^{2 \gamma}}{\Gamma^{2}(3+2 \gamma)} \frac{e^{\pi \alpha / \beta}}{\left(1-\beta^{2}\right)^{\gamma}} \\
& \times\left|\Gamma\left(1+\gamma+i \frac{\alpha}{\beta}\right)\right|^{2},
\end{aligned}
$$

where $\beta=k_{e} / E_{e}=\sqrt{E_{e}^{2}-m_{e}^{2}} / E_{e}$ is the electron velocity, $\gamma=\sqrt{1-\alpha^{2}}-1, r_{p}=0.841 \mathrm{fm}$ is the electric radius of the proton [33]. The correlation coefficient $b\left(E_{e}\right)$ is the Fierz interference term [34]. The structure and the value of the Fierz interference term may depend on interactions beyond the SM [34]. Information of a contemporary theoretical and experimental status of the Fierz interference term can be found in [35-39] (see also [40,41]).

We would like to notice that recently $[29,30]$ the correlation coefficients $T\left(E_{e}\right), S\left(E_{e}\right)$, and $U\left(E_{e}\right)$ have been investigated theoretically within the SM at the level of $10^{-3}$ by taking into account (i) the outer modelindependent $O(\alpha / \pi) \mathrm{RC}$, calculated to leading order (LO) in the large nucleon mass $m_{N}$ expansion; (ii) the
$O\left(E_{e} / m_{N}\right)$ corrections, caused by weak magnetism and proton recoil; and (iii) the corrections, caused by interactions beyond the SM [25], including the contributions of the second class currents or the $G$-odd correlations (as for $G$-parity invariance of strong interactions, we refer to the paper by Lee and Yang [42]) by Weinberg [43] (see also $[44,45]$ and $[12-14])$.

The paper is organized as follows. In Sec. II we give the analytical expressions for the correlation function $\zeta\left(E_{e}\right)$, which is responsible for the correct electron-energy spectrum of the neutron beta decay and correct value of the neutron lifetime, and the correlation coefficients $X\left(E_{e}\right)$ for $X=a, A, B, \ldots, T$ and $U$, including (i) the $O(\alpha / \pi)$ and $O\left(\alpha E_{e} / m_{N}\right) \sim 10^{-5} \mathrm{RC}$; (ii) the $O\left(E_{e} / m_{N}\right)$ and $O\left(E_{e}^{2} / m_{N}^{2}\right) \sim 10^{-5}$ corrections, caused by weak magnetism and proton recoil; and (iii) Wilkinson's corrections of order of a few parts of $10^{-5}$, which we have calculated in Appendixes A, B and C. The results, represented in Sec. II, illustrate the SM theoretical description of the neutron beta decay at a level of $10^{-5}$ with a theoretical accuracy of a few parts of $10^{-6}$. In Sec. III we discuss the obtained results and some problems of the analysis of the contributions of the neutron radiative beta decay. In Appendixes A, B, C, and $\mathrm{D}$ we give detailed calculations of the correlation function $\zeta\left(E_{e}\right)$ and correlation coefficients $X\left(E_{e}\right)$ for $X=$ $a, A, B, \ldots, T$ and $U$ adduced in Sec. II. In Appendix E we give the analytical expressions of the correlation function $\zeta\left(E_{e}\right)$ and correlation coefficients $X\left(E_{e}\right)$ for $X=$ $a, A, B, \ldots, U$ as functions of the electron energy $E_{e}$ and the axial coupling constant $g_{A}$. For the practical applications and numerical analysis the correlation function $\zeta\left(E_{e}\right)$ and correlation coefficients $X\left(E_{e}\right)$ for $X=$ $a, A, B, \ldots, U$ are programmed in [46]. In Appendix $\mathrm{F}$ we give the contributions to the electron-energy and angular distribution of the neutron beta decay with correlation structures, which go beyond the standard correlation 
structures in Eq. (1) by Jackson et al. [25-27] and Ebel and Feldman [28].

\section{CORRELATION FUNCTIONS AND COEFFICIENTS OF THE ELECTRON-ENERGY AND ANGULAR DISTRIBUTION EQ. (1)}

In Appendixes A, B, C, and D at the level of $10^{-5}$ with a theoretical accuracy of a few parts of $10^{-6}$ we give a detailed SM calculation of the correlation function $\zeta\left(E_{e}\right)$ and correlation coefficients in Eq. (1), the correlation structures of which are invariant under time-reversal transformation, i.e., T-even. According to our analysis carried out in Appendixes A, B, C, and D, the correlation function $\zeta\left(E_{e}\right)$ and correlation coefficients can be represented in the following form:

$$
\begin{array}{r}
\zeta\left(E_{e}\right)=\zeta\left(E_{e}\right)_{\mathrm{RC}}+\zeta\left(E_{e}\right)_{\mathrm{RC}-\mathrm{PhV}}+\zeta\left(E_{e}\right)_{\mathrm{WP}}+\zeta\left(E_{e}\right)_{\mathrm{WC}}, \\
X\left(E_{e}\right)=X\left(E_{e}\right)_{\mathrm{RC}}+X\left(E_{e}\right)_{\mathrm{RC}-\mathrm{PhV}}+X\left(E_{e}\right)_{\mathrm{WP}}+X\left(E_{e}\right)_{\mathrm{WC}},
\end{array}
$$

where $X=a, A, B, K_{n}, Q_{n}, G, H, N, K_{e}, Q_{e}, S, T$, and $U$. Then, $Y\left(E_{e}\right)_{\mathrm{RC}}, Y\left(E_{e}\right)_{\mathrm{RC}-\mathrm{PhV}}, Y\left(E_{e}\right)_{\mathrm{WP}}$, and $Y\left(E_{e}\right)_{\mathrm{W}}$ for
$Y=\zeta, X$ are (i) the sum of the outer $O(\alpha / \pi) \mathrm{RC}$, calculated to LO in the large nucleon mass $m_{N}$ expansion, and $O\left(\alpha E_{e} / m_{N}\right) \sim 10^{-5} \mathrm{RC}$, which are treated as NLO corrections in the large nucleon mass $m_{N}$ expansion to the outer and inner $O(\alpha / \pi) \mathrm{RC}$ and denoted as $Y_{\mathrm{RC}-\mathrm{NLO}}$ (see Appendix A); (ii) the outer $O\left(\alpha E_{e} / m_{N}\right) \mathrm{RC}$, caused by the outer $O(\alpha / \pi) \mathrm{RC}$ and the phase-volume of the neutron beta decay taken to NLO in the large nucleon mass expansion; (iii) the sum of the $O\left(E_{e} / m_{N}\right)$ and $O\left(E_{e}^{2} / m_{N}^{2}\right) \sim 10^{-5}$ corrections, caused by weak magnetism and proton recoil; and (iv) Wilkinson's corrections of order $10^{-5}$. For the practical applications and numerical analysis the analytical expressions of the correlation function $\zeta\left(E_{e}\right)$ and correlation coefficients $X\left(E_{e}\right)$ for $X=a, A, B, K_{n}, Q_{n}, G, H, N$, $K_{e}, Q_{e}, S, T$, and $U$ are programmed in [46].

In order to illustrate the SM description of the neutron beta decay at the level $10^{-5}$ we represent the correlation function $\zeta\left(E_{e}\right)$ and correlation coefficients $a\left(E_{e}\right), A\left(E_{e}\right)$, $B\left(E_{e}\right), \ldots, U\left(E_{e}\right)$ with the contributions of the corrections, caused by weak magnetism and proton recoil of order $O\left(E_{e} / m_{N}\right)$ and $O\left(E_{e}^{2} / m_{N}^{2}\right)$ in Appendix B, and Wilkinson's corrections in Appendix $\mathrm{C}$, as functions of the variable $E_{e} / E_{0}$. According to our calculation in [46], we get

$$
\begin{aligned}
\zeta\left(E_{e}\right) & =\zeta\left(E_{e}\right)_{\mathrm{RC}}+\zeta\left(E_{e}\right)_{\mathrm{RC}-\mathrm{PhV}}-5.57 \times 10^{-4} \frac{E_{0}}{E_{e}}-3.20 \times 10^{-3}+9.81 \times 10^{-3} \frac{E_{e}}{E_{0}}+7.13 \times 10^{-5} \frac{E_{e}^{2}}{E_{0}^{2}}-3.16 \times 10^{-5} \frac{E_{e}}{\beta E_{0}}, \\
a\left(E_{e}\right) & =a\left(E_{e}\right)_{\mathrm{RC}}+a\left(E_{e}\right)_{\mathrm{RC}-\mathrm{PhV}}-5.81 \times 10^{-5} \frac{E_{0}}{E_{e}}+3.24 \times 10^{-3}-9.16 \times 10^{-3} \frac{E_{e}}{E_{0}}-3.16 \times 10^{-5} \frac{E_{0}-E_{e}}{\beta^{3} E_{0}}, \\
A\left(E_{e}\right) & =A\left(E_{e}\right)_{\mathrm{RC}}-6.71 \times 10^{-5} \frac{E_{0}}{E_{e}}-1.75 \times 10^{-3} \frac{E_{e}}{E_{0}}, \\
B\left(E_{e}\right)= & B\left(E_{e}\right)_{\mathrm{RC}}+5.26 \times 10^{-5} \frac{E_{0}}{E_{e}}+3.27 \times 10^{-4}-3.42 \times 10^{-4} \frac{E_{e}}{E_{0}}-2.87 \times 10^{-5} \frac{E_{e}^{2}}{E_{0}^{2}}, \\
K_{n}\left(E_{e}\right)= & K_{n}\left(E_{e}\right)_{\mathrm{RC}}+7.15 \times 10^{-4} \frac{E_{e}}{E_{0}}+1.51 \times 10^{-5} \frac{E_{e}^{2}}{E_{0}^{2}}, \\
Q_{n}\left(E_{e}\right)= & Q_{n}\left(E_{e}\right)_{\mathrm{RC}-\mathrm{PhV}}+3.19 \times 10^{-3}-7.29 \times 10^{-3} \frac{E_{e}}{E_{0}}-1.97 \times 10^{-5} \frac{E_{e}^{2}}{E_{0}^{2}}-3.12 \times 10^{-5} \frac{E_{0}-E_{e}}{\beta^{3} E_{0}}, \\
G\left(E_{e}\right)= & G\left(E_{e}\right)_{\mathrm{RC}}-5.59 \times 10^{-4} \frac{E_{0}}{E_{e}}+3.78 \times 10^{-4}-5.26 \times 10^{-5} \frac{E_{e}}{E_{0}}-1.26 \times 10^{-4} \frac{E_{e}^{2}}{E_{0}^{2}}, \\
H\left(E_{e}\right)= & H\left(E_{e}\right)_{\mathrm{RC}}+2.28 \times 10^{-5} \frac{E_{0}^{2}}{E_{e}^{2}}-1.28 \times 10^{-3} \frac{E_{0}}{E_{e}}+1.13 \times 10^{-3}-1.46 \times 10^{-5} \frac{E_{e}}{E_{0}}, \\
N\left(E_{e}\right)= & N\left(E_{e}\right)_{\mathrm{RC}}+2.33 \times 10^{-5} \frac{E_{0}^{2}}{E_{e}^{2}}-3.21 \times 10^{-4} \frac{E_{0}}{E_{e}}+5.73 \times 10^{-5}, \\
Q_{e}\left(E_{e}\right)= & Q_{e}\left(E_{e}\right)_{\mathrm{RC}}+Q_{e}\left(E_{e}\right)_{\mathrm{RC}-\mathrm{PhV}}+2.24 \times 10^{-5} \frac{E_{0}}{E_{e}}+7.00 \times 10^{-4}+3.57 \times 10^{-3} \frac{E_{e}}{E_{0}}-1.57 \times 10^{-5} \frac{E_{e}^{2}}{E_{0}^{2}} \\
& +1.04 \times 10^{-4}\left(1+\sqrt{1-\beta^{2}}\right) \frac{E_{0}-E_{e}}{\beta^{3} E_{0}},
\end{aligned}
$$




$$
\begin{aligned}
K_{e}\left(E_{e}\right)= & K_{e}\left(E_{e}\right)_{\mathrm{RC}}+K_{e}\left(E_{e}\right)_{\mathrm{RC}-\mathrm{PhV}}+1.93 \times 10^{-5} \frac{E_{0}}{E_{e}}-6.93 \times 10^{-4}+9.17 \times 10^{-3} \frac{E_{e}}{E_{0}}+2.95 \times 10^{-5} \frac{E_{e}^{2}}{E_{0}^{2}} \\
& +3.16 \times 10^{-5}\left(1+\sqrt{1-\beta^{2}}\right) \frac{E_{0}-E_{e}}{\beta^{3} E_{0}} \\
S\left(E_{e}\right)= & S\left(E_{e}\right)_{\mathrm{RC}}-2.82 \times 10^{-4} \\
T\left(E_{e}\right)= & T\left(E_{e}\right)_{\mathrm{RC}}-1.81 \times 10^{-4} \frac{E_{0}}{E_{e}}-1.25 \times 10^{-5}+2.77 \times 10^{-4} \frac{E_{e}}{E_{0}}+4.69 \times 10^{-5} \frac{E_{e}^{2}}{E_{0}^{2}}, \\
U\left(E_{e}\right)= & U\left(E_{e}\right)_{\mathrm{RC}}+1.19 \times 10^{-5}
\end{aligned}
$$

where the numerical coefficients are calculated at the axial coupling constant $g_{A}=1.2764$ [5]. The contributions of the corrections of the $O\left(\alpha E_{e} / m_{N}\right)$ RC are plotted in [46].

Then, the analytical expressions of $\zeta\left(E_{e}\right)_{\mathrm{RC}}$ and $X\left(E_{e}\right)_{\mathrm{RC}}$ for $X=a, A, \ldots, T$ and $U$ are given in Appendix A [see Eq. (A20)] and in [46]. At $\alpha=0$ the correlation function $\zeta\left(E_{e}\right)_{\mathrm{RC}}$ and the correlation coefficients $X\left(E_{e}\right)_{\mathrm{RC}}$ for $X=$ $a, A, \ldots, U$ reduce to their values, calculated to $\mathrm{LO}$ in the large nucleon mass $m_{N}$ expansion (see Appendix A). The outer $\mathrm{RC} \zeta\left(E_{e}\right)_{\mathrm{RC}-\mathrm{PhV}}$ and $X\left(E_{e}\right)_{\mathrm{RC}-\mathrm{PhV}}$ are calculated in Appendix D [see Eq. (D10)].

In addition to the correlation function $\zeta\left(E_{e}\right)$ and the correlation coefficients in Eq. (4) we give the correlation coefficient $A^{(\beta)}\left(E_{e}\right)=A\left(E_{e}\right)+\frac{1}{3} Q_{n}\left(E_{e}\right)$ [15] that measures the electron (beta) asymmetry of the neutron beta decay [5]:

$$
\begin{aligned}
A^{(\beta)}\left(E_{e}\right)= & A^{(\beta)}\left(E_{e}\right)_{\mathrm{RC}}+\frac{1}{3} Q_{n}\left(E_{e}\right)_{\mathrm{RC}-\mathrm{PhV}}-6.67 \times 10^{-5} \frac{E_{0}}{E_{e}} \\
& +9.78 \times 10^{-4}-4.18 \times 10^{-3} \frac{E_{e}}{E_{0}} \\
& -1.04 \times 10^{-5} \frac{E_{0}-E_{e}}{\beta^{3} E_{0}}
\end{aligned}
$$

calculated at $g_{A}=1.2764$ [5], where $A^{(\beta)}\left(E_{e}\right)_{\mathrm{RC}}=A\left(E_{e}\right)_{\mathrm{RC}}$, since $Q_{n}\left(E_{e}\right)_{\mathrm{RC}}=0$ [see Eq. (A20)]. The correlation function $\zeta\left(E_{e}\right)$ and the correlation coefficients $X\left(E_{e}\right)$ for $X=$ $a, A, B, \ldots, U$ in Eq. (4) and $A^{(\beta)}\left(E_{e}\right)$ in Eq. (5) describe the neutron beta decay for a polarized neutron, a polarized electron, and an unpolarized proton at the level of $10^{-5}$ in the framework of the SM with a theoretical accuracy of a few parts of $10^{-6}$.

\section{DISCUSSION}

We have given a SM theoretical description of the neutron beta decay for a polarized neutron, a polarized electron, and an unpolarized proton at the level of $10^{-5}$ with a theoretical accuracy of a few parts of $10^{-6}$. To the wellknown $O(\alpha / \pi)$ RC [19-23] (see also [11,12,14,29,30]) and $O\left(E_{e} / m_{N}\right)$ corrections [47] and [15,22,23] (see also $[11,12,14,29,30])$ we have added (i) the inner $O\left(\alpha E_{e} / m_{N}\right) \sim 10^{-5} \mathrm{RC}[16,17]$, which are treated as NLO corrections in the large nucleon mass $m_{N}$ expansion to Sirlin's outer and inner $O(\alpha / \pi) \mathrm{RC}$, calculated to LO in the large nucleon mass $m_{N}$ expansion; (ii) the outer $O\left(\alpha E_{e} / m_{N}\right) \sim 10^{-5} \mathrm{RC}$, induced by Sirlin's outer $O(\alpha / \pi)$ $\mathrm{RC}$ and the phase-volume of the neutron beta decay, calculated to NLO in the large nucleon mass $m_{N}$ expansion; (iii) the $O\left(E_{e}^{2} / m_{N}^{2}\right) \sim 10^{-5}$ corrections [24], caused by weak magnetism and proton recoil; and (iv) Wilkinson's corrections [15] (see also $[11,12,14]$ ) of order $10^{-5}$. As has been shown in Eq. (4), all of these corrections define the SM background of the theoretical description of the neutron beta decay at the level of $10^{-5}$ with a theoretical accuracy of about a few parts of $10^{-6}$ [46].

Having accepted the value of the axial coupling constant $g_{A}=1.2764[5,6]$, the correlation function $\zeta\left(E_{e}\right)$ and correlation coefficients, given in Eqs. (4) and (5), can be used as the SM theoretical background of the neutron beta decay for experimental searches of contributions of interactions beyond the SM with experimental uncertainties of a few parts of $10^{-5}[4,7,8]$ (see also [12,24]). Because of Wilkinson's corrections, induced by the proton recoil in the electron-proton final-state Coulomb interaction [see Eq. (C8) in Appendix C] and the outer $O\left(\alpha E_{e} / m_{N}\right) \mathrm{RC}$ [see Eq. (D10) in Appendix D], the correlation function $\zeta\left(E_{e}\right)$ and correlation coefficients in Eqs. (4) and (5) are well-defined in the experimental electron-energy region $0.811 \mathrm{MeV} \leq E_{e} \leq 1.211 \mathrm{MeV}$ [5].

In Appendix $\mathrm{E}$ we give the analytical expressions for the correlation function $\zeta\left(E_{e}\right)$ and correlation coefficients $X\left(E_{e}\right)$ for $X=a, A, B, \ldots, U$ as functions of the electron energy $E_{e}$ and the axial coupling constant $g_{A}$. These expressions can be used as a SM theoretical background for processing experimental data on the neutron lifetime, the electron-antineutrino angular correlations, and electron and antineutrino asymmetries with experimental uncertainties of about a few parts of $10^{-5}$. Such a SM theoretical background and experimental data, obtained with experimental uncertainties of about a few parts of $10^{-5}$, should allow one to improve the currently available experimental value of the axial coupling constant $g_{A}[5,6]$. They can also be used for searches of contributions of interactions beyond the SM in experiments with polarized neutrons and electrons [8]. 
We also have to emphasize that for the correct description of the neutron lifetime one has to add the inner radiative corrections $\Delta_{R}^{V}$ and $\Delta_{R}^{A}$ of order $O(\alpha / \pi)$, defined by the Feynman $\gamma W$-box diagrams, to the rates of the neutron beta decay and superallowed nuclear beta decays, which have been calculated to LO in the large nucleon mass $m_{N}$ expansion in [48-56]. These corrections are very important for the correct extraction of the value of the CKM matrix element $V_{u d}$.

Finally, we would like to discuss the problem of the removal of infrared divergences for the calculation of the outer RC in the neutron beta decay. Since the virtual photon exchange leads to the dependence of the amplitude of the neutron beta decay on the infrared cutoff $\mu$, which is an infinitesimal photon mass $\mu$ in the covariant regularization [19,57-61], one has to take into account the contribution of the neutron radiative beta decay [19,57-61]. For this aim the energy and angular distribution of the rate of the neutron radiative decay should be summed with the energy and angular distribution of the rate of the neutron beta decay [19,57-61]. In case of the investigation of the electron-energy and angular distribution of the neutron beta decay [see, for example, Eq. (1)], the standard procedure for the calculation of distributions for both the neutron beta decay and neutron radiative beta decay is to integrate, first, over the proton 3-momenta and then over the energy of the antineutrino [19,21-23,57-69] (see also $[11-14,29,30,40])$. In the rest frame of the neutron and after the integration of the proton 3-momentum, the latter appears in the distributions in the form $\vec{k}_{p}=-\vec{k}_{e}-\vec{k}_{\bar{\nu}_{e}}$ imposed by momentum conservation, where $\vec{k}_{e}$ and $\vec{k}_{\bar{\nu}_{e}}$ are 3-momenta of the electron and antineutrino, respectively. In this case for the calculation of the electron-energy and angular distribution of the neutron radiative beta decay the integration over directions of the photon 3-momentum takes into account correlations of the photon 3-momentum with 3-momenta of the electron and antineutrino and implicitly with the proton 3-momentum (for the details of these calculations we refer to $[11,12,14,29,30])$. The electron-energy and angular distribution Eq. (1) is usually used for the measurements of the electron (beta) asymmetry, which is characterized by the correlation coefficient $A^{(\beta)}\left(E_{e}\right)$ [5]. In these measurements the electron asymmetry defines the asymmetry of the emission of decay electrons relative to the neutron spin polarization into solid angles related by the polar angle $\theta \rightarrow \pi-\theta[5,70-73]$ (for the details of the calculation we refer to [11]). The electronenergy and angular distribution Eq. (1) can also be applied to the measurement of the antineutrino asymmetry, which is practically defined by the correlation coefficient $B\left(E_{e}\right)$. Formally, the antineutrino asymmetry $B_{\text {exp }}\left(E_{e}\right)$ defines the asymmetry of the emission of the antineutrino relative to the neutron spin polarization into solid angles related by the polar angle $\theta \rightarrow \pi-\theta$. However, in experiments [7,74-76] because of the electroneutrality of the antineutrino such an asymmetry is equivalent to the asymmetry of the emission of the electron-proton pairs into the solid angles related by the polar angle $\theta \rightarrow \pi-\theta$. For the first time, the asymmetry $B_{\exp }\left(E_{e}\right)$ has been calculated by Glück et al. $[66,69]$ in terms of the correlation coefficients $a\left(E_{e}\right), A\left(E_{e}\right)$, and $B\left(E_{e}\right)$ (for the details of the calculation we refer to [11]).

In turn, for the measurements of the electronantineutrino angular correlations [77-79] and the proton recoil asymmetry, defined by the correlation coefficient $C$ [80], one has to use the electron-proton-energy and angular distribution (or the proton-energy and angular distribution) [65-69]. For the calculation of the electron-proton-energy and angular distribution one has to integrate over the antineutrino 3-momentum. Then, having integrated over the electron energy one obtains the proton-energy and angular distribution (for the details of the calculation we refer to [11]). The same procedure should be used for the neutron radiative beta decay [11]. In case of the neutron radiative beta decay for the calculation of the electronproton-photon-energy and angular distribution one deals with direct photon-proton correlations [65-69]. However, as has been shown in [81], the contributions of these correlations do not destroy the radiative corrections, defined by the functions $(\alpha / \pi) \bar{g}_{n}\left(E_{e}\right)$ and $(\alpha / \pi) f_{n}\left(E_{e}\right)$. As has been found in [81], the contributions of the photonproton correlations in the neutron radiative beta decay to the proton recoil asymmetry $C$ are of order of $10^{-4}$. They make the contributions of the radiative corrections to the proton recoil asymmetry $C$ symmetric with respect to a change $A_{0} \leftrightarrow B_{0}$, where $A_{0}$ and $B_{0}$ are the correlation coefficients $A\left(E_{e}\right)$ and $B\left(E_{e}\right)$ calculated to LO in the large nucleon mass $m_{N}$ expansion [1,2]. They depend on the axial coupling constant only [see also Eq. (A16) in Appendix A]. We are planning to carry out the analysis of the electron-proton-energy and angular distributions of the neutron beta decay at the SM theoretical level of about $10^{-5}$ in our forthcoming publication.

We would like to note that for practical applications and numerical analysis of the correlation function $\zeta\left(E_{e}\right)$ and correlation coefficients $a\left(E_{e}\right), A\left(E_{e}\right), B\left(E_{e}\right), \ldots, U\left(E_{e}\right)$ we have programmed their analytical expressions in [46]. We have carried out numerical calculations for the axial coupling constant $g_{A}=1.2764$ [5] and plotted the $O\left(\alpha E_{e} / m_{N}\right)$ corrections.

\section{ACKNOWLEDGMENTS}

We thank Hartmut Abele for discussions stimulating this work. The work of A. N. I. was supported by the Austrian "Fonds zur Förderung der Wissenschaftlichen Forschung" (FWF) under Contracts No. P31702-N27 and No. P26636N20, and "Deutsche Förderungsgemeinschaft" (DFG) AB 128/5-2. The work of R. H. was supported by the Deutsche Forschungsgemeinschaft in the SFB/TR 55. The work of M. Wellenzohn was supported by the MA 23. 


\section{APPENDIX A: THE ELECTRON-ENERGY AND ANGULAR DISTRIBUTION OF THE NEUTRON BETA DECAY WITH THE ACCOUNT FOR THE RADIATIVE CORRECTIONS OF ORDER $O\left(\alpha E_{e} / m_{N}\right)$}

According to $[11,29,30]$, the electron-energy and angular distribution of the neutron beta decay for a polarized neutron, a polarized electron, and an unpolarized proton in Eq. (1) is determined by

$$
\begin{aligned}
\frac{d^{5} \lambda_{n}\left(E_{e}, \vec{k}_{e}, \vec{k}_{\bar{\nu}}, \vec{\xi}_{n}, \vec{\xi}_{e}\right)}{d E_{e} d \Omega_{e} d \Omega_{\bar{\nu}}}= & \left(1+3 g_{A}^{2}\right) \frac{\left|G_{V}\right|^{2}}{16 \pi^{5}}\left(E_{0}-E_{e}\right)^{2} \sqrt{E_{e}^{2}-m_{e}^{2}} E_{e} F\left(E_{e}, Z=1\right) \\
& \times \Phi_{n}\left(\vec{k}_{e}, \vec{k}_{\bar{\nu}}\right) \sum_{\mathrm{pol}} \frac{\left|M\left(n \rightarrow p e^{-} \bar{\nu}_{e}\right)\right|^{2}}{\left(1+3 g_{A}^{2}\right)\left|G_{V}\right|^{2} 64 m_{n}^{2} E_{e} E_{\bar{\nu}}},
\end{aligned}
$$

where we sum over polarizations of the massive fermions. The function $\Phi_{n}\left(\vec{k}_{e}, \vec{k}_{\bar{\nu}}\right)$ defines the contributions of the phasevolume of the neutron beta decay $[11,24]$. It is equal to $[11,24]$

$$
\Phi_{n}\left(\vec{k}_{e}, \vec{k}_{\bar{\nu}}\right)=1+3 \frac{E_{e}}{m_{N}}\left(1-\frac{\vec{k}_{e} \cdot \vec{k}_{\bar{\nu}}}{E_{e} E_{\bar{\nu}}}\right),
$$

taken to NLO in the large nucleon mass $m_{N}$ expansion. The amplitude of the neutron beta decay, taking into account the radiative corrections of order $O(\alpha / \pi)$ and $O\left(\alpha E_{e} / m_{N}\right)$, is defined by [11,16,17]

$$
\begin{aligned}
M\left(n \rightarrow p e^{-} \bar{\nu}_{e}\right)= & -2 m_{n} G_{V}\left\{\left(1+U_{1}\right)\left[\varphi_{p}^{\dagger} \varphi_{n}\right]\left[\bar{u}_{e} \gamma^{0}\left(1-\gamma^{5}\right) v_{\bar{\nu}}\right]+g_{A}\left(1+U_{2}\right)\left[\varphi_{p}^{\dagger} \vec{\sigma} \varphi_{n}\right] \cdot\left[\bar{u}_{e} \vec{\gamma}\left(1-\gamma^{5}\right) v_{\bar{\nu}}\right]\right. \\
& +U_{3}\left[\varphi_{p}^{\dagger} \varphi_{n}\right]\left[\bar{u}_{e}\left(1-\gamma^{5}\right) v_{\bar{\nu}}\right]+g_{A} U_{4}\left[\varphi_{p}^{\dagger} \vec{\sigma} \varphi_{n}\right] \cdot\left[\bar{u}_{e} \gamma^{0} \vec{\gamma}\left(1-\gamma^{5}\right) v_{\bar{\nu}}\right]+U_{5}\left[\varphi_{p}^{\dagger}\left(\vec{k}_{e} \cdot \vec{\sigma}\right) \varphi_{n}\right]\left[\bar{u}_{e}\left(1-\gamma^{5}\right) v_{\bar{\nu}}\right] \\
& +U_{6}\left[\varphi_{p}^{\dagger}\left(\vec{k}_{\bar{\nu}} \cdot \vec{\sigma}\right) \varphi_{n}\right]\left[\bar{u}_{e}\left(1-\gamma^{5}\right) v_{\bar{\nu}}\right]+U_{7}\left[\varphi_{p}^{\dagger}\left(\vec{k}_{e} \cdot \vec{\sigma}\right) \varphi_{n}\right]\left[\bar{u}_{e} \gamma^{0}\left(1-\gamma^{5}\right) v_{\bar{\nu}}\right] \\
& +U_{8}\left[\varphi_{p}^{\dagger}\left(\vec{k}_{e} \cdot \vec{\sigma}\right) \vec{\sigma} \varphi_{n}\right] \cdot\left[\bar{u}_{e} \vec{\gamma}\left(1-\gamma^{5}\right) v_{\bar{\nu}}\right] .
\end{aligned}
$$

The functions $U_{j}$ for $j=1,2, \ldots, 8$ are given by

$$
\begin{aligned}
U_{1} & =\frac{\alpha}{2 \pi}\left(f_{\beta_{c}^{-}}\left(E_{e}, \mu\right)+\frac{E_{e}}{m_{N}} f_{V}\left(E_{e}\right)+\bar{g}_{\mathrm{st}}\left(E_{e}\right)\right), \\
U_{2} & =\frac{\alpha}{2 \pi}\left(f_{\beta_{c}^{-}}\left(E_{e}, \mu\right)+\frac{E_{e}}{m_{N}} f_{A}\left(E_{e}\right)+\frac{5}{2} \frac{m_{N}^{2}}{M_{W}^{2}} \ell n \frac{M_{W}^{2}}{m_{N}^{2}}+\frac{1}{g_{A}} \bar{f}_{\mathrm{st}}\left(E_{e}\right)\right), \\
U_{3} & =\frac{\alpha}{2 \pi}\left(-\frac{\sqrt{1-\beta^{2}}}{2 \beta} \ell n\left(\frac{1+\beta}{1-\beta}\right)+\frac{E_{e}}{m_{N}} f_{S}\left(E_{e}\right)\right), \\
U_{4} & =\frac{\alpha}{2 \pi}\left(-\frac{\sqrt{1-\beta^{2}}}{2 \beta} \ell n\left(\frac{1+\beta}{1-\beta}\right)+\frac{E_{e}}{m_{N}} f_{T}\left(E_{e}\right)\right), \\
U_{5} & =\frac{\alpha}{2 \pi} \frac{1}{E_{e}} \frac{E_{e}}{m_{N}} g_{S}\left(E_{e}\right), \quad U_{6}=\frac{\alpha}{2 \pi} \frac{1}{E_{e}} \frac{E_{e}}{m_{N}} h_{S}\left(E_{e}\right), \\
U_{7} & =\frac{\alpha}{2 \pi} \frac{1}{E_{e}} \frac{E_{e}}{m_{N}} g_{V}\left(E_{e}\right), \quad U_{8}=\frac{\alpha}{2 \pi} \frac{1}{E_{e}} \frac{E_{e}}{m_{N}} h_{A}\left(E_{e}\right),
\end{aligned}
$$

where $\beta=k_{e} / E_{e}=\sqrt{1-m_{e}^{2} / E_{e}^{2}}$ is the electron velocity, the function $f_{\beta_{c}^{-}}\left(E_{e}, \mu\right)$, where $\mu$ is a covariant infrared cutoff having a meaning of a photon mass [19,57,58], was calculated by Sirlin [19] to LO in the large nucleon $m_{N}$ expansion [for the details of the calculation of the function $f_{\beta_{c}^{-}}\left(E_{e}, \mu\right)$ we refer to [11] ]. It defines so-called outer model-independent radiative corrections [18]. Then, the functions $f_{V}\left(E_{e}\right), f_{A}\left(E_{e}\right), f_{S}\left(E_{e}\right), f_{T}\left(E_{e}\right), g_{V}\left(E_{e}\right), g_{S}\left(E_{e}\right), h_{S}\left(E_{e}\right)$, and $h_{A}\left(E_{e}\right)$ determine the inner radiative corrections dependent on the axial coupling constant $g_{A}$ to Sirlin's outer radiative corrections $O(\alpha / \pi)$, calculated to NLO in the large nucleon mass $m_{N}$ expansion in [16]. In turn, the functions $\bar{g}_{\mathrm{st}}\left(E_{e}\right)$ and $\bar{f}_{\mathrm{st}}\left(E_{e}\right)$ describe the inner radiative corrections, caused by the hadronic structure of the neutron and calculated to NLO in the large nucleon $m_{N}$ mass expansion in [17] as NLO corrections to Sirlin's inner radiative corrections $O(\alpha / \pi)$, caused by the hadronic structure of the neutron and calculated to LO in the large nucleon mass expansion $[19,20]$. The analytical expressions of these functions are equal to $[11,16,17]$ 


$$
\begin{aligned}
f_{\beta_{c}^{-}}\left(E_{e}, \mu\right) & =\frac{3}{4} \ell n \frac{m_{N}^{2}}{m_{e}^{2}}-\frac{11}{8}+\ell n\left(\frac{\mu}{m_{e}}\right)\left[\frac{1}{\beta} \ell n\left(\frac{1+\beta}{1-\beta}\right)-2\right]+\frac{1}{2 \beta} \ell n\left(\frac{1+\beta}{1-\beta}\right)-\frac{1}{4 \beta} \ell n^{2}\left(\frac{1+\beta}{1-\beta}\right)-\frac{1}{\beta} \operatorname{Li}_{2}\left(\frac{2 \beta}{1+\beta}\right), \\
f_{V}\left(E_{e}\right) & =1+\frac{1}{2} \ell n \frac{m_{N}^{2}}{m_{e}^{2}}+\frac{2-3 \beta^{2}}{2 \beta} \ell n\left(\frac{1+\beta}{1-\beta}\right)+\left(g_{A}-1\right)\left[-\frac{1}{4}-\frac{5}{8} \ell n \frac{m_{N}^{2}}{m_{e}^{2}}-\frac{2-5 \beta^{2}}{8 \beta} \ell n\left(\frac{1+\beta}{1-\beta}\right)\right], \\
f_{A}\left(E_{e}\right) & =1+\frac{1}{2} \ell n \frac{m_{N}^{2}}{m_{e}^{2}}+\frac{2-3 \beta^{2}}{2 \beta} \ell n\left(\frac{1+\beta}{1-\beta}\right)+\frac{g_{A}-1}{g_{A}}\left[\frac{3}{4} \ell n \frac{m_{N}^{2}}{m_{e}^{2}}-\frac{3}{4} \beta \ell n\left(\frac{1+\beta}{1-\beta}\right)\right], \\
f_{S}\left(E_{e}\right) & =\sqrt{1-\beta^{2}}\left\{-\frac{1}{2} \ell n \frac{m_{N}^{2}}{m_{e}^{2}}+\frac{2 E_{0}-E_{e}}{E_{e}} \frac{1}{2 \beta} \ell n\left(\frac{1+\beta}{1-\beta}\right)+\left(g_{A}-1\right)\left[\frac{1}{4}-\frac{1}{8} \ell n \frac{m_{N}^{2}}{m_{e}^{2}}-\frac{1}{4 \beta} \ell n\left(\frac{1+\beta}{1-\beta}\right)\right]\right\}, \\
f_{T}\left(E_{e}\right) & =\sqrt{1-\beta^{2}}\left[-\frac{1}{2} \ell n \frac{m_{N}^{2}}{m_{e}^{2}}+\frac{1}{2 \beta} \ell n\left(\frac{1+\beta}{1-\beta}\right)\right], \\
g_{S}\left(E_{e}\right) & =\left(g_{A}-1\right) \frac{\sqrt{1-\beta^{2}}}{8 \beta} \ell n\left(\frac{1+\beta}{1-\beta}\right), \\
h_{S}\left(E_{e}\right) & =\frac{\sqrt{1-\beta^{2}}}{\beta} \ell n\left(\frac{1+\beta}{1-\beta}\right), \\
g_{V}\left(E_{e}\right) & =-\frac{1}{2} \ell n \frac{m_{N}^{2}}{m_{e}^{2}}+\frac{1}{2 \beta} \ell n\left(\frac{1+\beta}{1-\beta}\right)+\left(g_{A}-1\right)\left[-\frac{1}{4}-\frac{3}{8} \ell n \frac{m_{N}^{2}}{m_{e}^{2}}+\frac{5}{8 \beta} \ell n\left(\frac{1+\beta}{1-\beta}\right)\right], \\
h_{A}\left(E_{e}\right) & =-\frac{1}{2} \ell n \frac{m_{N}^{2}}{m_{e}^{2}}+\frac{1}{2 \beta} \ell n\left(\frac{1+\beta}{1-\beta}\right),
\end{aligned}
$$

where $\operatorname{Li}_{2}(z)$ is the polylogarithmic function [82], and

$$
\begin{aligned}
& \bar{g}_{\mathrm{st}}\left(E_{e}\right)=-G_{\mathrm{st}}^{(V)} \frac{E_{0}}{m_{N}}+\left(G_{\mathrm{st}}^{(W)}+F_{\mathrm{st}}^{(W)}\right) \frac{m_{N}^{2}}{M_{W}^{2}}+H_{\mathrm{st}}^{(V)} \frac{E_{e}}{m_{N}}=0.098\left(1+0.95 \frac{E_{e}}{E_{0}}\right), \\
& \bar{f}_{\mathrm{st}}\left(E_{e}\right)=+G_{\mathrm{st}}^{(A)} \frac{E_{0}}{m_{N}}-H_{\mathrm{st}}^{(W)} \frac{m_{N}^{2}}{M_{W}^{2}}-H_{\mathrm{st}}^{(A)} \frac{E_{e}}{m_{N}}=0.057\left(1+\frac{E_{e}}{E_{0}}\right),
\end{aligned}
$$

where $E_{0}=\left(m_{n}^{2}-m_{p}^{2}+m_{e}^{2}\right) / 2 m_{n}=1.2926 \mathrm{MeV}$ is the end-point energy of the electron-energy spectrum [1,2], calculated for $m_{n}=939.9564 \mathrm{MeV}, m_{p}=938.2721 \mathrm{MeV}$, and $m_{e}=0.5110 \mathrm{MeV}$ [10]. Then, $m_{N}=\left(m_{n}+m_{p}\right) / 2=$ $938.9188 \mathrm{MeV}$ and $M_{W}=80.379 \mathrm{GeV}$ are nucleon and electroweak $W^{-}$-boson masses [10], respectively. The structure constants $G_{\mathrm{st}}^{(V)}$ and so on, calculated in [17], are equal to $G_{\mathrm{st}}^{(V)}=-70.71, H_{\mathrm{st}}^{(V)}=67.75, G_{\mathrm{st}}^{(W)}=8.94, G_{\mathrm{st}}^{(A)}=41.95$, $H_{\mathrm{st}}^{(A)}=-40.78, H_{\mathrm{st}}^{(W)}=2.10$, and $F_{\mathrm{st}}^{(W)}=-1.64$. For the subsequent analysis of radiative corrections we follow [29] (see also [11]) and represent the function $f_{\beta_{c}^{-}}\left(E_{e}, \mu\right)$ as follows:

$$
f_{\beta_{c}^{-}}\left(E_{e}, \mu\right)=\bar{g}_{n}\left(E_{e}\right)+\frac{1-\beta^{2}}{2 \beta} \ell n \frac{1+\beta}{1-\beta}-g_{\beta \gamma}^{(1)}\left(E_{e}, \mu\right),
$$

where $2 \bar{g}_{n}\left(E_{e}\right)$ is Sirlin's function, defining the outer radiative corrections of order $O(\alpha / \pi)$ to the neutron lifetime [19]. The function $g_{\beta \gamma}^{(1)}\left(E_{e}, \mu\right)$ can be removed by the contribution of the neutron radiative beta decay $n \rightarrow p+e^{-}+\bar{\nu}_{e}+\gamma$ with a real photon $\gamma$, which should be added, according to Berman [57] and Kinoshita and Sirlin [58] (see also Sirlin [19]), for the removal of the dependence of the neutron lifetime on the infrared cutoff. For the detailed calculation of the function $g_{\beta \gamma}^{(1)}\left(E_{e}, \mu\right)$ as well as the function $g_{\beta \gamma}^{(1)}\left(E_{e}, \omega_{\min }\right)$, describing the contributions of the neutron radiative beta decay $n \rightarrow$ $p+e^{-}+\bar{\nu}_{e}+\gamma$ to the neutron lifetime, where $\omega_{\min }$ is a noncovariant infrared cutoff having a meaning of the photonenergy threshold of the detector, we refer to [11]. We adduce here the analytical expressions of these functions for completeness (see [11]) 


$$
\begin{aligned}
g_{\beta \gamma}^{(1)}\left(E_{e}, \mu\right)= & {\left[\ln \left(\frac{2\left(E_{0}-E_{e}\right)}{\mu}\right)-\frac{3}{2}+\frac{1}{3} \frac{E_{0}-E_{e}}{E_{e}}\left(1+\frac{1}{8} \frac{E_{0}-E_{e}}{E_{e}}\right)\right]\left[\frac{1}{\beta} \ln \left(\frac{1+\beta}{1-\beta}\right)-2\right] } \\
& +1+\frac{1}{2 \beta} \ln \left(\frac{1+\beta}{1-\beta}\right)-\frac{1}{4 \beta} \ln \left(\frac{1+\beta}{1-\beta}\right)-\frac{1}{\beta} \operatorname{Li}_{2}\left(\frac{2 \beta}{1+\beta}\right)+\frac{1}{12} \frac{\left(E_{0}-E_{e}\right)^{2}}{E_{e}^{2}}, \\
g_{\beta \gamma}^{(1)}\left(E_{e}, \omega_{\min }\right)= & {\left[\ln \left(\frac{E_{0}-E_{e}}{\omega_{\min }}\right)-\frac{3}{2}+\frac{1}{3} \frac{E_{0}-E_{e}}{E_{e}}\left(1+\frac{1}{8} \frac{E_{0}-E_{e}}{E_{e}}\right)\right]\left[\frac{1}{\beta} \ln \left(\frac{1+\beta}{1-\beta}\right)-2\right]+\frac{1}{12} \frac{\left(E_{0}-E_{e}\right)^{2}}{E_{e}^{2}} . }
\end{aligned}
$$

The Hermitian conjugate amplitude of the neutron beta decay Eq. (A3) is equal to

$$
\begin{aligned}
M^{\dagger}\left(n \rightarrow p e^{-} \bar{\nu}_{e}\right)= & -2 m_{n} G_{V}\left\{\left(1+U_{1}\right)\left[\varphi_{n}^{\dagger} \varphi_{p}\right]\left[\bar{v}_{\bar{\nu}} \gamma^{0}\left(1-\gamma^{5}\right) u_{e}\right]+g_{A}\left(1+U_{2}\right)\left[\varphi_{n}^{\dagger} \vec{\sigma} \varphi_{p}\right] \cdot\left[\bar{v}_{\bar{\nu}} \vec{\gamma}\left(1-\gamma^{5}\right) u_{e}\right]\right. \\
& +U_{3}\left[\varphi_{n}^{\dagger} \varphi_{p}\right]\left[\bar{v}_{\bar{\nu}}\left(1+\gamma^{5}\right) u_{e}\right]-g_{A} U_{4}\left[\varphi_{p}^{\dagger} \vec{\sigma} \varphi_{n}\right] \cdot\left[\bar{v}_{\bar{\nu}} \gamma^{0} \vec{\gamma}\left(1+\gamma^{5}\right) u_{e}\right]+U_{5}\left[\varphi_{n}^{\dagger}\left(\vec{k}_{e} \cdot \vec{\sigma}\right) \varphi_{p}\right]\left[\bar{v}_{\bar{\nu}}\left(1+\gamma^{5}\right) u_{e}\right] \\
& +U_{6}\left[\varphi_{n}^{\dagger}\left(\vec{k}_{\bar{\nu}} \cdot \vec{\sigma}\right) \varphi_{p}\right]\left[\bar{v}_{\bar{\nu}}\left(1+\gamma^{5}\right) u_{e}\right]+U_{7}\left[\varphi_{n}^{\dagger}\left(\vec{k}_{e} \cdot \vec{\sigma}\right) \varphi_{p}\right]\left[\bar{v}_{\bar{\nu}} \gamma^{0}\left(1-\gamma^{5}\right) u_{e}\right] \\
& \left.+U_{8}\left[\varphi_{n}^{\dagger}\left(\vec{k}_{e} \cdot \vec{\sigma}\right) \vec{\sigma} \varphi_{p}\right] \cdot\left[\bar{v}_{\bar{\nu}} \vec{\gamma}\left(1-\gamma^{5}\right) u_{e}\right]\right\} .
\end{aligned}
$$

We use this amplitude for the calculation of the square of absolute value of the amplitude Eq. (A3), summed over polarizations of massive particles. It is equal to

$$
\begin{aligned}
& \sum_{\mathrm{pol}} \frac{\left|M\left(n \rightarrow p e^{-} \bar{\nu}_{e}\right)\right|^{2}}{\left(1+3 g_{A}^{2}\right)\left|G_{V}\right|^{2} 64 m_{n}^{2} E_{e} E_{\bar{\nu}}} \\
& =\frac{1}{\left(1+3 g_{A}^{2}\right) 8 E_{e} E_{\bar{\nu}}}\left\{\left(1+2 U_{1}\right) \operatorname{tr}\left\{\left(1+\vec{\xi}_{n} \cdot \vec{\sigma}\right)\right\} \operatorname{tr}\left\{\left(\hat{k}_{e}+m_{e} \gamma^{5} \hat{\zeta}_{e}\right) \gamma^{0} \hat{k}_{\bar{\nu}} \gamma^{0}\left(1-\gamma^{5}\right)\right\}\right. \\
& +g_{A}\left(1+U_{1}+U_{2}\right) \operatorname{tr}\left\{\left(1+\vec{\xi}_{n} \cdot \vec{\sigma}\right) \vec{\sigma}\right\} \cdot \operatorname{tr}\left\{\left(\hat{k}_{e}+m_{e} \gamma^{5} \hat{\zeta}_{e}\right) \vec{\gamma} \hat{k}_{\bar{\nu}} \gamma^{0}\left(1-\gamma^{5}\right)\right\}+g_{A}\left(1+U_{1}+U_{2}\right) \operatorname{tr}\left\{\left(1+\vec{\xi}_{n} \cdot \vec{\sigma}\right) \vec{\sigma}\right\} \\
& \cdot \operatorname{tr}\left\{\left(\hat{k}_{e}+m_{e} \gamma^{5} \hat{\zeta}_{e}\right) \gamma^{0} \hat{k}_{\bar{\nu}} \vec{\gamma}\left(1-\gamma^{5}\right)\right\}+g_{A}^{2}\left(1+2 U_{2}\right) \operatorname{tr}\left\{\left(1+\vec{\xi}_{n} \cdot \vec{\sigma}\right) \sigma^{a} \sigma^{b}\right\} \operatorname{tr}\left\{\left(\hat{k}_{e}+m_{e} \gamma^{5} \hat{\zeta}_{e}\right) \gamma^{b} \hat{k}_{\bar{\nu}} \gamma^{a}\left(1-\gamma^{5}\right)\right\} \\
& +U_{3} \operatorname{tr}\left\{\left(1+\vec{\xi}_{n} \cdot \vec{\sigma}\right)\right\} \operatorname{tr}\left\{\left(m_{e}+\hat{k}_{e} \gamma^{5} \hat{\zeta}_{e}\right) \hat{k}_{\bar{\nu}} \gamma^{0}\left(1-\gamma^{5}\right)\right\}+U_{3} \operatorname{tr}\left\{\left(1+\vec{\xi}_{n} \cdot \vec{\sigma}\right)\right\} \operatorname{tr}\left\{\left(m_{e}+\hat{k}_{e} \gamma^{5} \hat{\zeta}_{e}\right) \gamma^{0} \hat{k}_{\bar{\nu}}\left(1+\gamma^{5}\right)\right\} \\
& +g_{A} U_{4} \operatorname{tr}\left\{\left(1+\vec{\xi}_{n} \cdot \vec{\sigma}\right) \vec{\sigma}\right\} \cdot \operatorname{tr}\left\{\left(m_{e}+\hat{k}_{e} \gamma^{5} \hat{\zeta}_{e}\right) \gamma^{0} \vec{\gamma}_{\bar{\nu}} \gamma^{0}\left(1-\gamma^{5}\right)\right\}-g_{A} U_{4} \operatorname{tr}\left\{\left(1+\vec{\xi}_{n} \cdot \vec{\sigma}\right) \vec{\sigma}\right\} \cdot \operatorname{tr}\left\{\left(m_{e}+\hat{k}_{e} \gamma^{5} \hat{\zeta}_{e}\right)\right. \\
& \left.\times \gamma^{0} \hat{k}_{\bar{\nu}} \gamma^{0} \vec{\gamma}\left(1+\gamma^{5}\right)\right\}+U_{5} \operatorname{tr}\left\{\left(1+\vec{\xi}_{n} \cdot \vec{\sigma}\right)\left(\vec{k}_{e} \cdot \vec{\sigma}\right)\right\} \operatorname{tr}\left\{\left(m_{e}+\hat{k}_{e} \gamma^{5} \hat{\zeta}_{e}\right) \hat{k}_{\bar{\nu}} \gamma^{0}\left(1-\gamma^{5}\right)\right\}+U_{5} \operatorname{tr}\left\{\left(1+\vec{\xi}_{n} \cdot \vec{\sigma}\right)\left(\vec{k}_{e} \cdot \vec{\sigma}\right)\right\} \\
& \times \operatorname{tr}\left\{\left(m_{e}+\hat{k}_{e} \gamma^{5} \hat{\zeta}_{e}\right) \gamma^{0} \hat{k}_{\bar{\nu}}\left(1+\gamma^{5}\right)\right\}+U_{6} \operatorname{tr}\left\{\left(1+\vec{\xi}_{n} \cdot \vec{\sigma}\right)\left(\vec{k}_{\bar{\nu}} \cdot \vec{\sigma}\right)\right\} \operatorname{tr}\left\{\left(m_{e}+\hat{k}_{e} \gamma^{5} \hat{\zeta}_{e}\right) \hat{k}_{\bar{\nu}} \gamma^{0}\left(1-\gamma^{5}\right)\right\}+U_{6} \operatorname{tr}\left\{\left(1+\vec{\xi}_{n} \cdot \vec{\sigma}\right)\right. \\
& \left.\times\left(\vec{k}_{e} \cdot \vec{\sigma}\right)\right\} \operatorname{tr}\left\{\left(m_{e}+\hat{k}_{e} \gamma^{5} \hat{\zeta}_{e}\right) \gamma^{0} \hat{k}_{\bar{\nu}}\left(1+\gamma^{5}\right)\right\}+U_{7} \operatorname{tr}\left\{\left(1+\vec{\xi}_{n} \cdot \vec{\sigma}\right)\left(\vec{k}_{e} \cdot \vec{\sigma}\right)\right\} \operatorname{tr}\left\{\left(\hat{k}_{e}+m_{e} \gamma^{5} \hat{\zeta}_{e}\right) \gamma^{0} \hat{k}_{\bar{\nu}} \gamma^{0}\left(1-\gamma^{5}\right)\right\} \\
& +U_{7} \operatorname{tr}\left\{\left(1+\vec{\xi}_{n} \cdot \vec{\sigma}\right)\left(\vec{k}_{e} \cdot \vec{\sigma}\right) \operatorname{tr}\left\{\left(\hat{k}_{e}+m_{e} \gamma^{5} \hat{\zeta}_{e}\right) \gamma^{0} \hat{k}_{\bar{\nu}} \gamma^{0}\left(1-\gamma^{5}\right)\right\}+U_{8} \operatorname{tr}\left\{\left(1+\vec{\xi}_{n} \cdot \vec{\sigma}\right)\left(\vec{k}_{e} \cdot \vec{\sigma}\right) \vec{\sigma}\right\} \cdot \operatorname{tr}\left\{\left(\hat{k}_{e}+m_{e} \gamma^{5} \hat{\zeta}_{e}\right)\right.\right. \\
& \left.\times \vec{\gamma} \hat{k}_{\bar{\nu}} \gamma^{0}\left(1-\gamma^{5}\right)\right\}+U_{8} \operatorname{tr}\left\{\left(1+\vec{\xi}_{n} \cdot \vec{\sigma}\right) \vec{\sigma}\left(\vec{k}_{e} \cdot \vec{\sigma}\right)\right\} \cdot \operatorname{tr}\left\{\left(\hat{k}_{e}+m_{e} \gamma^{5} \hat{\zeta}_{e}\right) \gamma^{0} \hat{k}_{\bar{\nu}} \vec{\gamma}\left(1-\gamma^{5}\right)\right\}+g_{A} U_{3} \operatorname{tr}\left\{\left(1+\vec{\xi}_{n} \cdot \vec{\sigma}\right) \vec{\sigma}\right\} \\
& \cdot \operatorname{tr}\left\{\left(m_{e}+\hat{k}_{e} \gamma^{5} \hat{\zeta}_{e}\right) \hat{k}_{\bar{\nu}} \vec{\gamma}\left(1-\gamma^{5}\right)\right\}+g_{A} U_{3} \operatorname{tr}\left\{\left(1+\vec{\xi}_{n} \cdot \vec{\sigma}\right) \vec{\sigma}\right\} \cdot \operatorname{tr}\left\{\left(m_{e}+\hat{k}_{e} \gamma^{5} \hat{\zeta}_{e}\right) \vec{\gamma} \hat{k}_{\bar{\nu}}\left(1+\gamma^{5}\right)\right\}+g_{A}^{2} U_{4} \operatorname{tr}\left\{\left(1+\vec{\xi}_{n} \cdot \vec{\sigma}\right)\right. \\
& \left.\times \sigma^{a} \sigma^{b}\right\} \operatorname{tr}\left\{\left(m_{e}+\hat{k}_{e} \gamma^{5} \hat{\zeta}_{e}\right) \gamma^{0} \gamma^{b} \hat{k}_{\bar{\nu}} \gamma^{a}\left(1-\gamma^{5}\right)\right\}-g_{A}^{2} U_{4} \operatorname{tr}\left\{\left(1+\vec{\xi}_{n} \cdot \vec{\sigma}\right) \sigma^{a} \sigma^{b}\right\} \operatorname{tr}\left\{\left(m_{e}+\hat{k}_{e} \gamma^{5} \hat{\zeta}_{e}\right) \gamma^{b} \hat{k}_{\bar{\nu}} \gamma^{0} \gamma^{a}\left(1-\gamma^{5}\right)\right\} \\
& +g_{A} U_{5} \operatorname{tr}\left\{\left(1+\vec{\xi}_{n} \cdot \vec{\sigma}\right) \vec{\sigma}\left(\vec{k}_{e} \cdot \vec{\sigma}\right)\right\} \cdot \operatorname{tr}\left\{\left(m_{e}+\hat{k}_{e} \gamma^{5} \hat{\zeta}_{e}\right) \hat{k}_{\bar{\nu}} \vec{\gamma}\left(1-\gamma^{5}\right)\right\}+g_{A} U_{5} \operatorname{tr}\left\{\left(1+\vec{\xi}_{n} \cdot \vec{\sigma}\right)\left(\vec{k}_{e} \cdot \vec{\sigma}\right) \vec{\sigma}\right\} \cdot \operatorname{tr}\left\{\left(m_{e}+\hat{k}_{e} \gamma^{5} \hat{\zeta}_{e}\right)\right. \\
& \left.\times \vec{\gamma} \hat{k}_{\bar{\nu}}\left(1+\gamma^{5}\right)\right\}+g_{A} U_{6} \operatorname{tr}\left\{\left(1+\vec{\xi}_{n} \cdot \vec{\sigma}\right) \vec{\sigma}\left(\vec{k}_{\bar{\nu}} \cdot \vec{\sigma}\right)\right\} \cdot \operatorname{tr}\left\{\left(m_{e}+\hat{k}_{e} \gamma^{5} \hat{\zeta}_{e}\right) \hat{k}_{\bar{\nu}} \vec{\gamma}\left(1-\gamma^{5}\right)\right\}+g_{A} U_{6} \operatorname{tr}\left\{\left(1+\vec{\xi}_{n} \cdot \vec{\sigma}\right)\left(\vec{k}_{\bar{\nu}} \cdot \vec{\sigma}\right) \vec{\sigma}\right\} \\
& \cdot \operatorname{tr}\left\{\left(m_{e}+\hat{k}_{e} \gamma^{5} \hat{\zeta}_{e}\right) \vec{\gamma}_{\bar{\nu}}\left(1+\gamma^{5}\right)\right\}+g_{A} U_{7} \operatorname{tr}\left\{\left(1+\vec{\xi}_{n} \cdot \vec{\sigma}\right) \vec{\sigma}\left(\vec{k}_{e} \cdot \vec{\sigma}\right)\right\} \cdot \operatorname{tr}\left\{\left(\hat{k}_{e}+m_{e} \gamma^{5} \hat{\zeta}_{e}\right) \gamma^{0} \hat{k}_{\bar{\nu}} \vec{\gamma}\left(1-\gamma^{5}\right)\right\}+g_{A} U_{7} \\
& \times \operatorname{tr}\left\{\left(1+\vec{\xi}_{n} \cdot \vec{\sigma}\right)\left(\vec{k}_{e} \cdot \vec{\sigma}\right) \vec{\sigma}\right\} \cdot \operatorname{tr}\left\{\left(\hat{k}_{e}+m_{e} \gamma^{5} \hat{\zeta}_{e}\right) \vec{\gamma}_{\bar{\nu}} \gamma^{0}\left(1-\gamma^{5}\right)\right\}+g_{A} U_{8} \operatorname{tr}\left\{\left(1+\vec{\xi}_{n} \cdot \vec{\sigma}\right) \sigma^{a}\left(\vec{k}_{e} \cdot \vec{\sigma}\right) \sigma^{b}\right\} \operatorname{tr}\left\{\left(\hat{k}_{e}+m_{e} \gamma^{5} \hat{\zeta}_{e}\right)\right. \\
& \left.\times \gamma^{b} \hat{k}_{\bar{\nu}} \gamma^{a}\left(1-\gamma^{5}\right)\right\}+g_{A} U_{8} \operatorname{tr}\left\{\left(1+\vec{\xi}_{n} \cdot \vec{\sigma}\right) \sigma^{a}\left(\vec{k}_{e} \cdot \vec{\sigma}\right) \sigma^{b}\right\} \operatorname{tr}\left\{\left(\hat{k}_{e}+m_{e} \gamma^{5} \hat{\zeta}_{e}\right) \gamma^{b} \hat{k}_{\bar{\nu}} \gamma^{a}\left(1-\gamma^{5}\right)\right\} .
\end{aligned}
$$

Having calculated the traces over the nucleon degrees of freedom and using the properties of the Dirac matrices [31]

$$
\gamma^{\alpha} \gamma^{\nu} \gamma^{\mu}=\gamma^{\alpha} \eta^{\nu \mu}-\gamma^{\nu} \eta^{\mu \alpha}+\gamma^{\mu} \eta^{\alpha \nu}+i \varepsilon^{\alpha \nu \mu \beta} \gamma_{\beta} \gamma^{5}
$$

and $\gamma^{\mu} \gamma^{\nu}+\gamma^{\nu} \gamma^{\mu}=2 \eta^{\mu \nu}$, where $\eta^{\mu \nu}$ is the metric tensor of the Minkowski spacetime, $\varepsilon^{\alpha \nu \mu \beta}$ is the Levi-Civita tensor defined by $\varepsilon^{0123}=1$ and $\varepsilon_{\alpha \nu \mu \beta}=-\varepsilon^{\alpha \nu \mu \beta}$ [31], we transcribe the right-hand side (RHS) of Eq. (A10) into the form [11,12,14] 


$$
\begin{aligned}
& \sum_{\text {pol }} \frac{\left|M\left(n \rightarrow p e^{-} \bar{\nu}_{e}\right)\right|^{2}}{\left(1+3 g_{A}^{2}\right)\left|G_{V}\right|^{2} 64 m_{n}^{2} E_{e} E_{\bar{\nu}}} \\
& =\frac{1}{4 E_{e}}\left\{\left[\left(1+\frac{2}{1+3 g_{A}^{2}}\left(U_{1}+3 g_{A}^{2} U_{2}\right)\right)+\left(B_{0}+\frac{2}{1+3 g_{A}^{2}}\left(g_{A}\left(U_{1}+U_{2}\right)+2 g_{A}^{2} U_{2}\right)\right)\right.\right. \\
& \left.\times \frac{\vec{\xi}_{n} \cdot \vec{k}_{\bar{\nu}}}{E_{\bar{\nu}}}+\frac{2 E_{e}}{1+3 g_{A}^{2}}\left(U_{7}-g_{A} U_{8}\right) \frac{\vec{\xi}_{n} \cdot \vec{k}_{e}}{E_{e}}+\frac{2 E_{e}}{1+3 g_{A}^{2}}\left(g_{A} U_{7}+\left(1-2 g_{A}\right) U_{8}\right) \frac{\vec{k}_{e} \cdot \vec{k}_{\bar{\nu}}}{E_{e} E_{\bar{\nu}}}\right] \operatorname{tr}\left\{\left(\hat{k}_{e}+m_{e} \gamma^{5} \hat{\zeta}_{e}\right) \gamma^{0}\left(1-\gamma^{5}\right)\right\} \\
& +\left[\left(a_{0}+\frac{2}{1+3 g_{A}^{2}}\left(U_{1}-g_{A}^{2} U_{2}\right)\right) \frac{\vec{k}_{\bar{\nu}}}{E_{\bar{\nu}}}+\left(A_{0}+\frac{2}{1+3 g_{A}^{2}}\left(g_{A}\left(U_{1}+U_{2}\right)-2 g_{A}^{2} U_{2}\right)\right) \vec{\xi}_{n}+\frac{2 E_{e}}{1+3 g_{A}^{2}}\left(U_{7}-g_{A} U_{8}\right) \frac{\left(\vec{\xi}_{n} \cdot \vec{k}_{e}\right)}{E_{e}} \frac{\vec{k}_{\bar{\nu}}}{E_{\bar{\nu}}}\right. \\
& +\frac{2 E_{e}}{1+3 g_{A}^{2}}\left(g_{A} U_{7}+\left(1+2 g_{A}\right) U_{8}\right) \frac{\vec{k}_{e}}{E_{e}}+\frac{2 E_{e}}{1+3 g_{A}^{2}}\left(-g_{A} U_{7}+\left(1+2 g_{A}\right) U_{8}\right) \frac{\left(\vec{k}_{e} \cdot \vec{k}_{\bar{\nu}}\right)}{E_{e} E_{\bar{\nu}}} \vec{\xi}_{n}+\frac{2 E_{e}}{1+3 g_{A}^{2}}\left(g_{A} U_{7}-\left(1-2 g_{A}\right)\right. \\
& \left.\left.\times U_{8}\right) \frac{\left(\vec{\xi}_{n} \cdot \vec{k}_{\bar{\nu}}\right)}{E_{\bar{\nu}}} \frac{\vec{k}_{e}}{E_{e}}\right] \cdot \operatorname{tr}\left\{\left(\hat{k}_{e}+m_{e} \gamma^{5} \hat{\zeta}_{e}\right) \vec{\gamma}\left(1-\gamma^{5}\right)\right\}+\left[\frac{2}{1+3 g_{A}^{2}}\left(U_{3}+3 g_{A}^{2} U_{4}+g_{A} E_{\bar{\nu}} U_{6}\right)+\frac{2}{1+3 g_{A}^{2}}\left(g_{A}\left(U_{3}+U_{4}\right)\right.\right. \\
& \left.\left.+2 g_{A}^{2} U_{4}+E_{\bar{\nu}} U_{6}\right) \frac{\vec{\xi}_{n} \cdot \vec{k}_{\bar{\nu}}}{E_{\bar{\nu}}}+\frac{2 E_{e}}{1+3 g_{A}^{2}} U_{5} \frac{\vec{\xi}_{n} \cdot \vec{k}_{e}}{E_{e}}+\frac{2 E_{e}}{1+3 g_{A}^{2}} g_{A} U_{5} \frac{\vec{k}_{e} \cdot \vec{k}_{\bar{\nu}}}{E_{e} E_{\bar{\nu}}}\right] \operatorname{tr}\left\{\left(m_{e}+\hat{k}_{e} \gamma^{5} \hat{\zeta}_{e}\right)\right\}+\left[-\frac{2 E_{e}}{1+3 g_{A}^{2}} g_{A} U_{5}\right. \\
& \times \frac{\left(\vec{\xi}_{n} \cdot \vec{k}_{e}\right) \vec{k}_{\bar{\nu}}}{E_{e} E_{\bar{\nu}}}-\frac{2 E_{\bar{\nu}}}{1+3 g_{A}^{2}}\left(1+g_{A}\right) U_{6} \frac{\left(\vec{\xi}_{n} \cdot \vec{k}_{\bar{\nu}}\right) \vec{k}_{\bar{\nu}}}{E_{\bar{\nu}}^{2}}+\frac{2}{1+3 g_{A}^{2}}\left(-g_{A}\left(U_{3}+U_{4}\right)+2 g_{A}^{2} U_{4}+g_{A} E_{\bar{\nu}} U_{6}\right) \vec{\xi}_{n}+\frac{2}{1+3 g_{A}^{2}} \\
& \left.\times\left(-U_{3}+g_{A}^{2} U_{4}-g_{A} E_{\bar{\nu}} U_{6}\right) \frac{\vec{k}_{\bar{\nu}}}{E_{\bar{\nu}}}-\frac{2 E_{e}}{1+3 g_{A}^{2}} g_{A} U_{5} \frac{\vec{k}_{e}}{E_{e}}+\frac{2 E_{e}}{1+3 g_{A}^{2}} g_{A} U_{5} \frac{\left(\vec{\xi}_{n} \cdot \vec{k}_{\bar{\nu}}\right) \vec{k}_{e}}{E_{e} E_{\bar{\nu}}}-\frac{2 E_{e}}{1+3 g_{A}^{2}} g_{A} U_{5} \frac{\left(\vec{k}_{e} \cdot \vec{k}_{\bar{\nu}}\right) \vec{\xi}_{n}}{E_{e} E_{\bar{\nu}}}\right] \\
& \cdot \operatorname{tr}\left\{\left(m_{e}+\hat{k}_{e} \gamma^{5} \hat{\zeta}_{e}\right) \gamma^{0} \vec{\gamma}^{5}\right\}+\left[\frac{2}{1+3 g_{A}^{2}} g_{A}\left(U_{3}-U_{4}-E_{\bar{\nu}} U_{6}\right) i \frac{\vec{\xi}_{n} \times \vec{k}_{\bar{\nu}}}{E_{\bar{\nu}}}-\frac{2 E_{e}}{1+3 g_{A}^{2}} g_{A} U_{5} i \frac{\vec{\xi}_{n} \times \vec{k}_{e}}{E_{e}}+\frac{2 E_{e}}{1+3 g_{A}^{2}} g_{A} U_{5}\right. \\
& \left.\left.\times i \frac{\vec{k}_{e} \times \vec{k}_{\bar{\nu}}}{E_{e} E_{\bar{\nu}}}\right] \cdot \operatorname{tr}\left\{\left(m_{e}+\hat{k}_{e} \gamma^{5} \hat{\zeta}_{e}\right) \gamma^{0} \vec{\gamma}\right\}\right\} \text {. }
\end{aligned}
$$

Having calculated the traces over leptonic degrees of freedom we arrive at the expression

$$
\begin{aligned}
\sum_{\mathrm{pol}} & \frac{\left|M\left(n \rightarrow p e^{-} \bar{\nu}_{e}\right)\right|^{2}}{\left(1+3 g_{A}^{2}\right)\left|G_{V}\right|^{2} 64 m_{n}^{2} E_{e} E_{\bar{\nu}}} \\
= & {\left[\left(1+\frac{2}{1+3 g_{A}^{2}}\left(U_{1}+3 g_{A}^{2} U_{2}\right)\right)+\left(B_{0}+\frac{2}{1+3 g_{A}^{2}}\left(g_{A}\left(U_{1}+U_{2}\right)+2 g_{A}^{2} U_{2}\right)\right) \frac{\vec{\xi}_{n} \cdot \vec{k}_{\bar{\nu}}}{E_{\bar{\nu}}}+\frac{2 E_{e}}{1+3 g_{A}^{2}}\left(U_{7}-g_{A} U_{8}\right) \frac{\vec{\xi}_{n} \cdot \vec{k}_{e}}{E_{e}}\right.} \\
& \left.+\frac{2 E_{e}}{1+3 g_{A}^{2}}\left(g_{A} U_{7}+\left(1-2 g_{A}\right) U_{8}\right) \frac{\vec{k}_{e} \cdot \vec{k}_{\bar{\nu}}}{E_{e} E_{\bar{\nu}}}\right]\left(1-\frac{m_{e}}{E_{e}} \zeta_{e}^{0}\right)+\left[\left(a_{0}+\frac{2}{1+3 g_{A}^{2}}\left(U_{1}-g_{A}^{2} U_{2}\right)\right) \frac{\vec{k}_{\bar{\nu}}}{E_{\bar{\nu}}}\right. \\
& +\left(A_{0}+\frac{2}{1+3 g_{A}^{2}}\left(g_{A}\left(U_{1}+U_{2}\right)-2 g_{A}^{2} U_{2}\right)\right) \vec{\xi}_{n}+\frac{2 E_{e}}{1+3 g_{A}^{2}}\left(U_{7}-g_{A} U_{8}\right) \frac{\left(\vec{\xi}_{n} \cdot \vec{k}_{e}\right)}{E_{e}} \frac{\vec{k}_{\bar{\nu}}}{E_{\bar{\nu}}}+\frac{2 E_{e}}{1+3 g_{A}^{2}}\left(g_{A} U_{7}+\left(1+2 g_{A}\right) U_{8}\right) \frac{\vec{k}_{e}}{E_{e}} \\
& \left.+\frac{2 E_{e}}{1+3 g_{A}^{2}}\left(-g_{A} U_{7}+\left(1+2 g_{A}\right) U_{8}\right) \frac{\left(\vec{k}_{e} \cdot \vec{k}_{\bar{\nu}}\right)}{E_{e} E_{\bar{\nu}}} \vec{\xi}_{n}+\frac{2 E_{e}}{1+3 g_{A}^{2}}\left(g_{A} U_{7}-\left(1-2 g_{A}\right) U_{8}\right) \frac{\left(\vec{\xi}_{n} \cdot \vec{k}_{\bar{\nu}}\right)}{E_{\bar{\nu}}} \frac{\vec{k}_{e}}{E_{e}}\right] \cdot\left(\frac{\vec{k}_{e}}{E_{e}}-\frac{m_{e}}{E_{e}} \vec{\zeta}_{e}\right) \\
& +\left[\frac{2}{1+3 g_{A}^{2}}\left(U_{3}+3 g_{A}^{2} U_{4}+g_{A} E_{\bar{\nu}} U_{6}\right)+\frac{2}{1+3 g_{A}^{2}}\left(g_{A}\left(U_{3}+U_{4}\right)+2 g_{A}^{2} U_{4}+E_{\bar{\nu}} U_{6}\right) \frac{\vec{\xi}_{n} \cdot \vec{k}_{\bar{\nu}}}{E_{\bar{\nu}}}+\frac{2 E_{e}}{1+3 g_{A}^{2}} U_{5} \frac{\vec{\xi}_{n} \cdot \vec{k}_{e}}{E_{e}}\right. \\
& \left.+\frac{2 E_{e}}{1+3 g_{A}^{2}} g_{A} U_{5} \frac{\vec{k}_{e} \cdot \vec{k}_{\bar{\nu}}}{E_{e} E_{\bar{\nu}}}\right] \frac{m_{e}}{E_{e}}+\left[-\frac{2 E_{e}}{1+3 g_{A}^{2}} g_{A} U_{5} \frac{\left(\vec{\xi}_{n} \cdot \vec{k}_{e}\right) \vec{k}_{\bar{\nu}}}{E_{e} E_{\bar{\nu}}}-\frac{2 E_{\bar{\nu}}}{1+3 g_{A}^{2}}\left(1+g_{A}\right) U_{6} \frac{\left(\vec{\xi}_{n} \cdot \vec{k}_{\bar{\nu}}\right) \vec{k}_{\bar{\nu}}}{E_{\bar{\nu}}^{2}}\right. \\
& +\frac{2}{1+3 g_{A}^{2}}\left(-g_{A}\left(U_{3}+U_{4}\right)+2 g_{A}^{2} U_{4}+g_{A} E_{\bar{\nu}} U_{6}\right) \vec{\xi}_{n}+\frac{2}{1+3 g_{A}^{2}}\left(-U_{3}+g_{A}^{2} U_{4}-g_{A} E_{\bar{\nu}} U_{6}\right) \frac{\vec{k}_{\bar{\nu}}}{E_{\bar{\nu}}}-\frac{2 E_{e}}{1+3 g_{A}^{2}} g_{A} U_{5} \frac{\vec{k}_{e}}{E_{e}}
\end{aligned}
$$




$$
\begin{aligned}
& \left.+\frac{2 E_{e}}{1+3 g_{A}^{2}} g_{A} U_{5} \frac{\left(\vec{\xi}_{n} \cdot \vec{k}_{\bar{\nu}}\right) \vec{k}_{e}}{E_{e} E_{\bar{\nu}}}-\frac{2 E_{e}}{1+3 g_{A}^{2}} g_{A} U_{5} \frac{\left(\vec{k}_{e} \cdot \vec{k}_{\bar{\nu}}\right) \vec{\xi}_{n}}{E_{e} E_{\bar{\nu}}}\right] \cdot\left(\vec{\zeta}_{e}-\frac{\vec{k}_{e}}{E_{e}} \zeta_{e}^{0}\right)+\left[\frac{2}{1+3 g_{A}^{2}} g_{A}\left(U_{3}-U_{4}-E_{\bar{\nu}} U_{6}\right)\right. \\
& \left.\times i \frac{\vec{\xi}_{n} \times \vec{k}_{\bar{\nu}}}{E_{\bar{\nu}}}-\frac{2 E_{e}}{1+3 g_{A}^{2}} g_{A} U_{5} i \frac{\vec{\xi}_{n} \times \vec{k}_{e}}{E_{e}}+\frac{2 E_{e}}{1+3 g_{A}^{2}} g_{A} U_{5} i \frac{\vec{k}_{e} \times \vec{k}_{\bar{\nu}}}{E_{e} E_{\bar{\nu}}}\right] \cdot i \frac{\vec{k}_{e} \times \vec{\xi}_{e}}{E_{e}} .
\end{aligned}
$$

In terms of the irreducible correlation structures the RHS of Eq. (A13) is given by

$$
\begin{aligned}
\sum_{\mathrm{pol}} \frac{\left|M\left(n \rightarrow p e^{-} \bar{\nu}_{e}\right)\right|^{2}}{\left(1+3 g_{A}^{2}\right)\left|G_{V}\right|^{2} 64 m_{n}^{2} E_{e} E_{\bar{\nu}}}= & \zeta\left(E_{e}\right)_{\mathrm{RC}}\left\{1+b\left(E_{e}\right)_{\mathrm{RC}} \frac{m_{e}}{E_{e}}+a\left(E_{e}\right)_{\mathrm{RC}} \frac{\vec{k}_{e} \cdot \vec{k}_{\bar{\nu}}}{E_{e} E_{\bar{\nu}}}+A\left(E_{e}\right)_{\mathrm{RC}} \frac{\vec{\xi}_{n} \cdot \vec{k}_{e}}{E_{e}}+B\left(E_{e}\right)_{\mathrm{RC}} \frac{\vec{\xi}_{n} \cdot \vec{k}_{\bar{\nu}}}{E_{\bar{\nu}}}\right. \\
& +K_{n}\left(E_{e}\right)_{\mathrm{RC}} \frac{\left(\vec{\xi}_{n} \cdot \vec{k}_{e}\right)\left(\vec{k}_{e} \cdot \vec{k}_{\bar{\nu}}\right)}{E_{e}^{2} E_{\bar{\nu}}}+G\left(E_{e}\right)_{\mathrm{RC}} \frac{\vec{\xi}_{e} \cdot \vec{k}_{e}}{E_{e}}+H\left(E_{e}\right)_{\mathrm{RC}} \frac{\vec{\xi}_{e} \cdot \vec{k}_{\bar{\nu}}}{E_{\bar{\nu}}}+N\left(E_{e}\right)_{\mathrm{RC}} \vec{\xi}_{n} \cdot \vec{\xi}_{e} \\
& +Q_{e}\left(E_{e}\right)_{\mathrm{RC}} \frac{\left(\vec{\xi}_{n} \cdot \vec{k}_{e}\right)\left(\vec{k}_{e} \cdot \vec{\xi}_{e}\right)}{\left(E_{e}+m_{e}\right) E_{e}}+K_{e}\left(E_{e}\right)_{\mathrm{RC}} \frac{\left(\vec{\xi}_{e} \cdot \vec{k}_{e}\right)\left(\vec{k}_{e} \cdot \vec{k}_{\bar{\nu}}\right)}{\left(E_{e}+m_{e}\right) E_{e} E_{\bar{\nu}}}+S\left(E_{e}\right)_{\mathrm{RC}} \frac{\left(\vec{\xi}_{n} \cdot \vec{\xi}_{e}\right)\left(\vec{k}_{e} \cdot \vec{k}_{\bar{\nu}}\right)}{E_{e} E_{\bar{\nu}}} \\
& +T\left(E_{e}\right)_{\mathrm{RC}} \frac{\left(\vec{\xi}_{n} \cdot \vec{k}_{\bar{\nu}}\right)\left(\vec{\xi}_{e} \cdot \vec{k}_{e}\right)}{E_{e} E_{\bar{\nu}}}+U\left(E_{e}\right)_{\mathrm{RC}} \frac{\left(\vec{\xi}_{n} \cdot \vec{k}_{e}\right)\left(\vec{\xi}_{e} \cdot \vec{k}_{\bar{\nu}}\right)}{E_{e} E_{\bar{\nu}}} \\
& +\frac{2 E_{e}}{1+3 g_{A}^{2}}\left(2 g_{A} U_{5}-\left(1-g_{A}\right) U_{7}-\left(1+g_{A}\right) U_{8}\right) \frac{\left(\vec{\xi}_{n} \cdot \vec{k}_{e}\right)\left(\vec{\xi}_{e} \cdot \vec{k}_{e}\right)\left(\vec{k}_{e} \cdot \vec{k}_{\bar{\nu}}\right)}{\left(E_{e}+m_{e}\right) E_{e}^{2} E_{\bar{\nu}}} \\
& -\frac{2 E_{e}}{1+3 g_{A}^{2}}\left(1+g_{A}\right) U_{6}\left[\left(\frac{\left(\vec{\xi}_{n} \cdot \vec{k}_{\bar{\nu}}\right)\left(\vec{\xi}_{e} \cdot \vec{k}_{\bar{\nu}}\right)}{E_{\bar{\nu}}^{2}}-\frac{1}{3} \vec{\xi}_{e} \cdot \vec{\xi}_{e}\right)\right. \\
& \left.\left.+\left(-\frac{\left(\vec{\xi}_{n} \cdot \vec{k}_{\bar{\nu}}\right)\left(\vec{\xi}_{e} \cdot \vec{k}_{e}\right)\left(\vec{k}_{e} \cdot \vec{k}_{\bar{\nu}}\right)}{\left(E_{e}+m_{e}\right) E_{e} E_{\bar{\nu}}^{2}}+\frac{1}{3} \frac{\left(\vec{\xi}_{n} \cdot \vec{k}_{2}\right)\left(\vec{\xi}_{e} \cdot \vec{k}_{e}\right)}{\left(E_{e}+m_{e}\right) E_{e}}\right)\right]\right\}
\end{aligned}
$$

where the index RC means that these corrections are defined by the outer radiative corrections of order $O(\alpha / \pi)$, calculated to LO in the large nucleon mass expansion [12,14,19,21], and the inner radiative corrections of order $O\left(\alpha E_{e} / m_{N}\right)[16,17]$. The correlation function $\zeta\left(E_{e}\right)_{\mathrm{RC}}$ and the correlation coefficients $\zeta\left(E_{e}\right)_{\mathrm{RC}} X\left(E_{e}\right)_{\mathrm{RC}}$ for $X=b, a, A, B, \ldots, T$ and $U$ are equal to

$$
\begin{aligned}
\zeta\left(E_{e}\right)_{\mathrm{RC}}= & \left(1+\frac{2}{1+3 g_{A}^{2}}\left(U_{1}+3 g_{A}^{2} U_{2}\right)\right)+\frac{2}{1+3 g_{A}^{2}}\left(U_{3}+3 g_{A}^{2} U_{4}+g_{A} E_{\bar{\nu}} U_{6}\right) \frac{m_{e}}{E_{e}} \\
& +\frac{2 E_{e}}{1+3 g_{A}^{2}}\left(g_{A} U_{7}+\left(1+2 g_{A}\right) U_{8}\right) \beta^{2}, \\
\zeta\left(E_{e}\right)_{\mathrm{RC}} b\left(E_{e}\right)_{\mathrm{RC}}= & 0 \\
\zeta\left(E_{e}\right)_{\mathrm{RC}} a\left(E_{e}\right)_{\mathrm{RC}}= & \left(a_{0}+\frac{2}{1+3 g_{A}^{2}}\left(U_{1}-g_{A}^{2} U_{2}\right)\right)+\frac{2 E_{e}}{1+3 g_{A}^{2}} g_{A} U_{5} \frac{m_{e}}{E_{e}}+\frac{2 E_{e}}{1+3 g_{A}^{2}}\left(g_{A} U_{7}+\left(1-2 g_{A}\right) U_{8}\right), \\
\zeta\left(E_{e}\right)_{\mathrm{RC}} A\left(E_{e}\right)_{\mathrm{RC}}= & \left(A_{0}+\frac{2}{1+3 g_{A}^{2}}\left(g_{A}\left(U_{1}+U_{2}\right)-2 g_{A}^{2} U_{2}\right)\right)+\frac{2 E_{e}}{1+3 g_{A}^{2}} \frac{m_{e}}{E_{e}} U_{5}+\frac{2 E_{e}}{1+3 g_{A}^{2}}\left(U_{7}-g_{A} U_{8}\right), \\
\zeta\left(E_{e}\right)_{\mathrm{RC}} B\left(E_{e}\right)_{\mathrm{RC}}= & \left(B_{0}+\frac{2}{1+3 g_{A}^{2}}\left(g_{A}\left(U_{1}+U_{2}\right)+2 g_{A}^{2} U_{2}\right)\right)+\frac{2}{1+3 g_{A}^{2}}\left(g_{A}\left(U_{3}+U_{4}\right)+2 g_{A}^{2} U_{4}+E_{\bar{\nu}} U_{6}\right) \frac{m_{e}}{E_{e}} \\
& +\frac{2 E_{e}}{1+3 g_{A}^{2}}\left(g_{A} U_{7}-\left(1-2 g_{A}\right) U_{8}\right) \beta^{2}, \\
\zeta\left(E_{e}\right)_{\mathrm{RC}} K_{n}\left(E_{e}\right)_{\mathrm{RC}}= & \frac{2 E_{e}}{1+3 g_{A}^{2}}\left(\left(1-g_{A}\right) U_{7}+\left(1+g_{A}\right) U_{8}\right),
\end{aligned}
$$




$$
\begin{aligned}
& \zeta\left(E_{e}\right)_{\mathrm{RC}} Q_{n}\left(E_{e}\right)_{\mathrm{RC}}=0, \\
& \zeta\left(E_{e}\right)_{\mathrm{RC}} G\left(E_{e}\right)_{\mathrm{RC}}=-\left(1+\frac{2}{1+3 g_{A}^{2}}\left(U_{1}+3 g_{A}^{2} U_{2}\right)\right)-\frac{2 E_{e}}{1+3 g_{A}^{2}} g_{A} U_{5} \frac{m_{e}}{E_{e}}-\frac{2 E_{e}}{1+3 g_{A}^{2}}\left(g_{A} U_{7}+\left(1+2 g_{A}\right) U_{8}\right), \\
& \zeta\left(E_{e}\right)_{\mathrm{RC}} H\left(E_{e}\right)_{\mathrm{RC}}=-\frac{m_{e}}{E_{e}}\left(a_{0}+\frac{2}{1+3 g_{A}^{2}}\left(U_{1}-g_{A}^{2} U_{2}\right)\right)+\frac{2}{1+3 g_{A}^{2}}\left(-U_{3}+g_{A}^{2} U_{4}-g_{A} E_{\bar{\nu}} U_{6}\right)+\frac{2 E_{e}}{1+3 g_{A}^{2}} g_{A} U_{5} \beta^{2}, \\
& \left.\zeta\left(E_{e}\right)_{\mathrm{RC}} N\left(E_{e}\right)_{\mathrm{RC}}=-\frac{m_{e}}{E_{e}}\left(A_{0}+\frac{2}{1+3 g_{A}^{2}}\left(g_{A}\left(U_{1}+U_{2}\right)-2 g_{A}^{2} U_{2}\right)\right)+\frac{2}{1+3 g_{A}^{2}}\left(-g_{A}\left(U_{3}+U_{4}\right)+2 g_{A}^{2}\right) U_{4}+g_{A} E_{\bar{\nu}} U_{6}\right) \\
& +\frac{2 E_{e}}{1+3 g_{A}^{2}} g_{A} U_{5} \beta^{2}-\frac{1}{3} \frac{2 E_{\bar{\nu}}}{1+3 g_{A}^{2}}\left(1+g_{A}\right) U_{6}, \\
& \zeta\left(E_{e}\right)_{\mathrm{RC}} Q_{e}\left(E_{e}\right)_{\mathrm{RC}}=-\left(A_{0}+\frac{2}{1+3 g_{A}^{2}}\left(g_{A}\left(U_{1}+U_{2}\right)-2 g_{A}^{2} U_{2}\right)\right)-\frac{2}{1+3 g_{A}^{2}}\left(-g_{A}\left(U_{3}+U_{4}\right)+2 g_{A}^{2} U_{4}+g_{A} E_{\bar{\nu}} U_{6}\right) \\
& +\frac{2}{1+3 g_{A}^{2}}\left(1+\frac{m_{e}}{E_{e}}\right)\left(g_{A} U_{5}-U_{7}+g_{A} U_{8}\right)+\frac{1}{3} \frac{2 E_{\bar{\nu}}}{1+3 g_{A}^{2}}\left(1+g_{A}\right) U_{6}, \\
& \zeta\left(E_{e}\right)_{\mathrm{RC}} K_{e}\left(E_{e}\right)_{\mathrm{RC}}=-\left(a_{0}+\frac{2}{1+3 g_{A}^{2}}\left(U_{1}-g_{A}^{2} U_{2}\right)\right)-\frac{2}{1+3 g_{A}^{2}}\left(-U_{3}+g_{A}^{2} U_{4}-g_{A} E_{\bar{\nu}} U_{6}\right)-\frac{2 E_{e}}{1+3 g_{A}^{2}}\left(g_{A} U_{5}+g_{A} U_{7}\right. \\
& \left.+\left(1-2 g_{A}\right) U_{8}\right)\left(1+\frac{m_{e}}{E_{e}}\right), \\
& \zeta\left(E_{e}\right)_{\mathrm{RC}} S\left(E_{e}\right)_{\mathrm{RC}}=-\frac{2}{1+3 g_{A}^{2}} g_{A}\left(U_{3}-U_{4}-E_{\bar{\nu}} U_{6}\right)-\frac{2 E_{e}}{1+3 g_{A}^{2}} g_{A} U_{5}-\frac{2 E_{e}}{1+3 g_{A}^{2}}\left(-g_{A} U_{7}+\left(1+2 g_{A}\right) U_{8}\right) \frac{m_{e}}{E_{e}}, \\
& \zeta\left(E_{e}\right)_{\mathrm{RC}} T\left(E_{e}\right)_{\mathrm{RC}}=-\left(B_{0}+\frac{2}{1+3 g_{A}^{2}}\left(g_{A}\left(U_{1}+U_{2}\right)+2 g_{A}^{2} U_{2}\right)\right)+\frac{2 E_{e}}{1+3 g_{A}^{2}}\left(g_{A} U_{5}-g_{A} U_{7}+\left(1-2 g_{A}\right) U_{8}\right), \\
& \zeta\left(E_{e}\right)_{\mathrm{RC}} U\left(E_{e}\right)_{\mathrm{RC}}=\frac{2}{1+3 g_{A}^{2}} g_{A}\left(U_{3}-U_{4}-E_{\bar{\nu}} U_{6}\right)-\frac{2 E_{e}}{1+3 g_{A}^{2}} g_{A} U_{5}-\frac{2 E_{e}}{1+3 g_{A}^{2}}\left(U_{7}-g_{A} U_{8}\right) \frac{m_{e}}{E_{e}},
\end{aligned}
$$

where the correlation coefficients $a_{0}, A_{0}$, and $B_{0}$ depend only on the axial coupling constant $g_{A}[1,2]$ (see also [29])

$$
a_{0}=\frac{1-g_{A}^{2}}{1+3 g_{A}^{2}}, \quad A_{0}=2 \frac{g_{A}\left(1-g_{A}\right)}{1+3 g_{A}^{2}}, \quad B_{0}=2 \frac{g_{A}\left(1+g_{A}\right)}{1+3 g_{A}^{2}}
$$

Using the definitions of the functions $U_{j}$ for $j=1,2, \ldots, 8$ in Eqs. (A4) and (A7) we transcribe Eq. (A15) as follows:

$$
\begin{aligned}
\zeta\left(E_{e}\right)_{\mathrm{RC}}= & 1+\frac{\alpha}{\pi}\left(\bar{g}_{n}\left(E_{e}\right)-g_{\beta \gamma}^{(1)}\left(E_{e}, \mu\right)\right)+\frac{1}{1+3 g_{A}^{2}} \frac{\alpha}{\pi} \frac{E_{e}}{m_{N}}\left(f_{V}\left(E_{e}\right)+\sqrt{1-\beta^{2}} f_{S}\left(E_{e}\right)+g_{A} g_{V}\left(E_{e}\right) \beta^{2}\right. \\
& \left.+\left(1+2 g_{A}\right) h_{A}\left(E_{e}\right) \beta^{2}+g_{A} \frac{E_{0}-E_{e}}{E_{e}} \sqrt{1-\beta^{2}} h_{S}\left(E_{e}\right)+3 g_{A}^{2} f_{A}\left(E_{e}\right)+3 g_{A}^{2} \sqrt{1-\beta^{2}} f_{T}\left(E_{e}\right)\right)+\frac{3 g_{A}^{2}}{1+3 g_{A}^{2}} \\
& \times \frac{\alpha}{\pi} \frac{5}{2} \frac{m_{N}^{2}}{M_{W}^{2}} \ell n \frac{M_{W}^{2}}{m_{N}^{2}}+\frac{1}{1+3 g_{A}^{2}} \frac{\alpha}{\pi}\left(\bar{g}_{\mathrm{st}}\left(E_{e}\right)+3 g_{A} \bar{f}_{\mathrm{st}}\left(E_{e}\right)\right),
\end{aligned}
$$

$\zeta\left(E_{e}\right)_{\mathrm{RC}} b\left(E_{e}\right)_{\mathrm{RC}}=0$,

$$
\begin{aligned}
\zeta\left(E_{e}\right)_{\mathrm{RC}} a\left(E_{e}\right)_{\mathrm{RC}}= & a_{0}\left(1+\frac{\alpha}{\pi}\left(\bar{g}_{n}\left(E_{e}\right)+\frac{1-\beta^{2}}{2 \beta} \ell n\left(\frac{1+\beta}{1-\beta}\right)-g_{\beta \gamma}^{(1)}\left(E_{e}, \mu\right)\right)\right)+\frac{1}{1+3 g_{A}^{2}} \frac{\alpha}{\pi} \frac{E_{e}}{m_{N}}\left(f_{V}\left(E_{e}\right)\right. \\
& \left.+g_{A} \sqrt{1-\beta^{2}} g_{S}\left(E_{e}\right)+g_{A} g_{V}\left(E_{e}\right)+\left(1-2 g_{A}\right) h_{A}\left(E_{e}\right)-g_{A}^{2} f_{A}\left(E_{e}\right)\right)-\frac{g_{A}^{2}}{1+3 g_{A}^{2}} \frac{\alpha 5}{\pi} \frac{m_{N}^{2}}{M_{W}^{2}} \ell n \frac{M_{W}^{2}}{m_{N}^{2}}+\frac{1}{1+3 g_{A}^{2}} \\
& \times \frac{\alpha}{\pi}\left(\bar{g}_{\mathrm{st}}\left(E_{e}\right)-g_{A} \bar{f}_{\mathrm{st}}\left(E_{e}\right)\right),
\end{aligned}
$$




$$
\begin{aligned}
& \zeta\left(E_{e}\right)_{\mathrm{RC}} A\left(E_{e}\right)_{\mathrm{RC}}=A_{0}\left(1+\frac{\alpha}{\pi}\left(\bar{g}_{n}\left(E_{e}\right)+\frac{1-\beta^{2}}{2 \beta} \ell n\left(\frac{1+\beta}{1-\beta}\right)-g_{\beta \gamma}^{(1)}\left(E_{e}, \mu\right)\right)\right)+\frac{1}{1+3 g_{A}^{2}} \frac{\alpha}{\pi} \frac{E_{e}}{m_{N}}\left(g_{A} f_{V}\left(E_{e}\right)\right. \\
& \left.+\sqrt{1-\beta^{2}} g_{S}\left(E_{e}\right)+g_{V}\left(E_{e}\right)-g_{A} h_{A}\left(E_{e}\right)+g_{A}\left(1-2 g_{A}\right) f_{A}\left(E_{e}\right)\right)+\frac{1}{1+3 g_{A}^{2}} g_{A}\left(1-2 g_{A}\right) \frac{\alpha}{\pi} \frac{5}{2} \frac{m_{N}^{2}}{M_{W}^{2}} \ell n \frac{M_{W}^{2}}{m_{N}^{2}} \\
& +\frac{1}{1+3 g_{A}^{2}} \frac{\alpha}{\pi}\left(g_{A} \bar{g}_{\mathrm{st}}\left(E_{e}\right)+\left(1-2 g_{A}\right) \bar{f}_{\mathrm{st}}\left(E_{e}\right)\right) \\
& \zeta\left(E_{e}\right)_{\mathrm{RC}} B\left(E_{e}\right)_{\mathrm{RC}}=B_{0}\left(1+\frac{\alpha}{\pi}\left(\bar{g}_{n}\left(E_{e}\right)-g_{\beta \gamma}^{(1)}\left(E_{e}, \mu\right)\right)\right)+\frac{1}{1+3 g_{A}^{2}} \frac{\alpha}{\pi} \frac{E_{e}}{m_{N}}\left(g_{A} f_{V}\left(E_{e}\right)+g_{A} \sqrt{1-\beta^{2}} f_{S}\left(E_{e}\right)\right. \\
& +\frac{E_{0}-E_{e}}{E_{e}} \sqrt{1-\beta^{2}} h_{S}\left(E_{e}\right)+g_{A} g_{V}\left(E_{e}\right) \beta^{2}-\left(1-2 g_{A}\right) h_{A}\left(E_{e}\right) \beta^{2}+g_{A}\left(1+2 g_{A}\right) f_{A}\left(E_{e}\right) \\
& \left.+g_{A}\left(1+2 g_{A}\right) \sqrt{1-\beta^{2}} f_{T}\left(E_{e}\right)\right)+\frac{1}{1+3 g_{A}^{2}} g_{A}\left(1+2 g_{A}\right) \frac{\alpha}{\pi} \frac{5}{2} \frac{m_{N}^{2}}{M_{W}^{2}} \ln \frac{M_{W}^{2}}{m_{N}^{2}} \\
& +\frac{1}{1+3 g_{A}^{2}} \frac{\alpha}{\pi}\left(g_{A} \bar{g}_{\mathrm{st}}\left(E_{e}\right)+\left(1+2 g_{A}\right) \bar{f}_{\mathrm{st}}\left(E_{e}\right)\right) \\
& \zeta\left(E_{e}\right)_{\mathrm{RC}} K_{n}\left(E_{e}\right)_{\mathrm{RC}}=\frac{1}{1+3 g_{A}^{2}} \frac{\alpha}{\pi} \frac{E_{e}}{m_{N}}\left(\left(1-g_{A}\right) g_{V}\left(E_{e}\right)+\left(1+2 g_{A}\right) h_{A}\left(E_{e}\right)\right), \\
& \zeta\left(E_{e}\right)_{\mathrm{RC}} Q_{n}\left(E_{e}\right)_{\mathrm{RC}}=0, \\
& \zeta\left(E_{e}\right)_{\mathrm{RC}} G\left(E_{e}\right)_{\mathrm{RC}}=-\left(1+\frac{\alpha}{\pi}\left(\bar{g}_{n}\left(E_{e}\right)+\frac{1-\beta^{2}}{2 \beta} \ell n\left(\frac{1+\beta}{1-\beta}\right)-g_{\beta \gamma}^{(1)}\left(E_{e}, \mu\right)\right)\right)-\frac{1}{1+3 g_{A}^{2}} \frac{\alpha}{\pi} \frac{E_{e}}{m_{N}}\left(f_{V}\left(E_{e}\right)+g_{A}\right. \\
& \left.\times \sqrt{1-\beta^{2}} g_{S}\left(E_{e}\right)+g_{A} g_{V}\left(E_{e}\right)+\left(1+2 g_{A}\right) h_{A}\left(E_{e}\right)+3 g_{A}^{2} f_{A}\left(E_{e}\right)\right)-\frac{3 g_{A}^{2}}{1+3 g_{A}^{2}} \frac{\alpha}{\pi} \frac{5}{2} \frac{m_{N}^{2}}{M_{W}^{2}} \ln \frac{M_{W}^{2}}{m_{N}^{2}} \\
& -\frac{1}{1+3 g_{A}^{2}} \frac{\alpha}{\pi}\left(\bar{g}_{\mathrm{st}}\left(E_{e}\right)+3 g_{A} \bar{f}_{\mathrm{st}}\left(E_{e}\right)\right) \\
& \zeta\left(E_{e}\right)_{\mathrm{RC}} H\left(E_{e}\right)_{\mathrm{RC}}=-\frac{m_{e}}{E_{e}} a_{0}\left(1+\frac{\alpha}{\pi}\left(\bar{g}_{n}\left(E_{e}\right)-\frac{\beta}{2} \ell n\left(\frac{1+\beta}{1-\beta}\right)-g_{\beta \gamma}^{(1)}\left(E_{e}, \mu\right)\right)\right)-\frac{1}{1+3 g_{A}^{2}} \frac{\alpha}{\pi} \frac{E_{e}}{m_{N}}\left(\sqrt{1-\beta^{2}} f_{V}\left(E_{e}\right)\right. \\
& \left.+f_{S}\left(E_{e}\right)+g_{A} g_{S}\left(E_{e}\right) \beta^{2}+g_{A} \frac{E_{0}-E_{e}}{E_{e}} h_{S}\left(E_{e}\right)-g_{A}^{2} \sqrt{1-\beta^{2}} f_{A}\left(E_{e}\right)-g_{A}^{2} f_{T}\left(E_{e}\right)\right) \\
& +\sqrt{1-\beta^{2}} \frac{g_{A}^{2}}{1+3 g_{A}^{2}} \frac{\alpha}{\pi} \frac{5}{2} \frac{m_{N}^{2}}{M_{W}^{2}} \ln \frac{M_{W}^{2}}{m_{N}^{2}}-\frac{1}{1+3 g_{A}^{2}} \frac{\alpha}{\pi} \sqrt{1-\beta^{2}}\left(\bar{g}_{\mathrm{st}}\left(E_{e}\right)-g_{A} \bar{f}_{\mathrm{st}}\left(E_{e}\right)\right), \\
& \zeta\left(E_{e}\right)_{\mathrm{RC}} N\left(E_{e}\right)_{\mathrm{RC}}=-\frac{m_{e}}{E_{e}} A_{0}\left(1+\frac{\alpha}{\pi}\left(\bar{g}_{n}\left(E_{e}\right)-\frac{\beta}{2} \ell n\left(\frac{1+\beta}{1-\beta}\right)-g_{\beta \gamma}^{(1)}\left(E_{e}, \mu\right)\right)\right)-\frac{1}{1+3 g_{A}^{2}} \frac{\alpha}{\pi} \frac{E_{e}}{m_{N}}\left(g_{A} f_{V}\left(E_{e}\right)\right. \\
& \times \sqrt{1-\beta^{2}}+g_{A} f_{S}\left(E_{e}\right)-g_{A} g_{S}\left(E_{e}\right) \beta^{2}+\frac{1}{3}\left(1-2 g_{A}\right) \frac{E_{0}-E_{e}}{E_{e}} h_{S}\left(E_{e}\right)+g_{A}\left(1-2 g_{A}\right) \sqrt{1-\beta^{2}} f_{A}\left(E_{e}\right) \\
& \left.+g_{A}\left(1-2 g_{A}\right) f_{T}\left(E_{e}\right)\right)-\sqrt{1-\beta^{2}} \frac{1}{1+3 g_{A}^{2}} g_{A}\left(1-2 g_{A}\right) \frac{\alpha}{\pi} \frac{5}{2} \frac{m_{N}^{2}}{M_{W}^{2}} \ln \frac{M_{W}^{2}}{m_{N}^{2}} \\
& -\frac{1}{1+3 g_{A}^{2}} \frac{\alpha}{\pi} \sqrt{1-\beta^{2}}\left(g_{A} \bar{g}_{\mathrm{st}}\left(E_{e}\right)+\left(1-2 g_{A}\right) \bar{f}_{\mathrm{st}}\left(E_{e}\right)\right) \text {, }
\end{aligned}
$$




$$
\begin{aligned}
& \zeta\left(E_{e}\right)_{\mathrm{RC}} Q_{e}\left(E_{e}\right)_{\mathrm{RC}}=-A_{0}\left(1+\frac{\alpha}{\pi}\left(\bar{g}_{n}\left(E_{e}\right)+\left(1+\sqrt{1-\beta^{2}}\right) \frac{\sqrt{1-\beta^{2}}}{2 \beta} \ell n\left(\frac{1+\beta}{1-\beta}\right)-g_{\beta \gamma}^{(1)}\left(E_{e}, \mu\right)\right)\right) \\
& -\frac{1}{1+3 g_{A}^{2}} \frac{\alpha}{\pi} \frac{E_{e}}{m_{N}}\left(g_{A} f_{V}\left(E_{e}\right)-g_{A} f_{S}\left(E_{e}\right)-g_{A}\left(1+\sqrt{1-\beta^{2}} g_{S}\left(E_{e}\right)+\left(1+\sqrt{1-\beta^{2}}\right) g_{V}\left(E_{e}\right)\right.\right. \\
& \left.-\frac{1}{3}\left(1-2 g_{A}\right) \frac{E_{0}-E_{e}}{E_{e}} h_{S}\left(E_{e}\right)+g_{A}\left(1-2 g_{A}\right) f_{A}\left(E_{e}\right)-g_{A}\left(1-2 g_{A}\right) f_{T}\left(E_{e}\right)\right) \\
& -\frac{1}{1+3 g_{A}^{2}} g_{A}\left(1-2 g_{A}\right) \frac{\alpha}{\pi} \frac{5}{2} \frac{m_{N}^{2}}{M_{W}^{2}} \ell n \frac{M_{W}^{2}}{m_{N}^{2}}-\frac{1}{1+3 g_{A}^{2}} \frac{\alpha}{\pi}\left(g_{A} \bar{g}_{\mathrm{st}}\left(E_{e}\right)+\left(1-2 g_{A}\right) \bar{f}_{\mathrm{st}}\left(E_{e}\right)\right), \\
& \zeta\left(E_{e}\right)_{\mathrm{RC}} K_{e}\left(E_{e}\right)_{\mathrm{RC}}=-a_{0}\left(1+\frac{\alpha}{\pi}\left(\bar{g}_{n}\left(E_{e}\right)+\left(1+\sqrt{1-\beta^{2}}\right) \frac{\sqrt{1-\beta^{2}}}{2 \beta} \ell n\left(\frac{1+\beta}{1-\beta}\right)-g_{\beta \gamma}^{(1)}\left(E_{e}, \mu\right)\right)\right) \\
& -\frac{1}{1+3 g_{A}^{2}} \frac{\alpha}{\pi} \frac{E_{e}}{m_{N}}\left(f_{V}\left(E_{e}\right)-f_{S}\left(E_{e}\right)+g_{A}\left(1+\sqrt{1-\beta^{2}}\right) g_{S}\left(E_{e}\right)+g_{A}\left(1+\sqrt{1-\beta^{2}}\right) g_{V}\left(E_{e}\right)\right. \\
& \left.+\left(1-2 g_{A}\right)\left(1+\sqrt{1-\beta^{2}}\right) h_{A}\left(E_{e}\right)-g_{A} \frac{E_{0}-E_{e}}{E_{e}} h_{S}\left(E_{e}\right)-g_{A}^{2} f_{A}\left(E_{e}\right)+g_{A}^{2} f_{T}\left(E_{e}\right)\right) \\
& +\frac{g_{A}^{2}}{1+3 g_{A}^{2}} \frac{\alpha}{\pi} \frac{5}{2} \frac{m_{N}^{2}}{M_{W}^{2}} \ell n \frac{M_{W}^{2}}{m_{N}^{2}}-\frac{1}{1+3 g_{A}^{2}} \frac{\alpha}{\pi}\left(\bar{g}_{\mathrm{st}}\left(E_{e}\right)-g_{A} \bar{f}_{\mathrm{st}}\left(E_{e}\right)\right), \\
& \zeta\left(E_{e}\right)_{\mathrm{RC}} S\left(E_{e}\right)_{\mathrm{RC}}=-\frac{1}{1+3 g_{A}^{2}} \frac{\alpha}{\pi} \frac{E_{e}}{m_{N}}\left(g_{A} f_{S}\left(E_{e}\right)-g_{A} f_{T}\left(E_{e}\right)+g_{A} g_{S}\left(E_{e}\right)-g_{A} \frac{E_{0}-E_{e}}{E_{e}} h_{S}\left(E_{e}\right)\right. \\
& \left.-g_{A} \sqrt{1-\beta^{2}} g_{V}\left(E_{e}\right)+\left(1+2 g_{A}\right) \sqrt{1-\beta^{2}} h_{A}\left(E_{e}\right)\right) \text {, } \\
& \zeta\left(E_{e}\right)_{\mathrm{RC}} T\left(E_{e}\right)_{\mathrm{RC}}=-B_{0}\left(1+\frac{\alpha}{\pi}\left(\bar{g}_{n}\left(E_{e}\right)+\frac{1-\beta^{2}}{2 \beta} \ell n\left(\frac{1+\beta}{1-\beta}\right)-g_{\beta \gamma}^{(1)}\left(E_{e}, \mu\right)\right)\right) \\
& -\frac{1}{1+3 g_{A}^{2}} \frac{\alpha}{\pi} \frac{E_{e}}{m_{N}}\left(g_{A} f_{V}\left(E_{e}\right)-g_{A} g_{S}\left(E_{e}\right)+g_{A} g_{V}\left(E_{e}\right)-\left(1-2 g_{A}\right) h_{A}\left(E_{e}\right)+g_{A}\left(1+2 g_{A}\right) f_{A}\left(E_{e}\right)\right) \\
& -\frac{1}{1+3 g_{A}^{2}} g_{A}\left(1+2 g_{A}\right) \frac{\alpha}{\pi} \frac{5}{2} \frac{m_{N}^{2}}{M_{W}^{2}} \ell n \frac{M_{W}^{2}}{m_{N}^{2}}-\frac{1}{1+3 g_{A}^{2}} \frac{\alpha}{\pi}\left(g_{A} \bar{g}_{\mathrm{st}}\left(E_{e}\right)+\left(1+2 g_{A}\right) \bar{f}_{\mathrm{st}}\left(E_{e}\right)\right), \\
& \zeta\left(E_{e}\right)_{\mathrm{RC}} U\left(E_{e}\right)_{\mathrm{RC}}=\frac{1}{1+3 g_{A}^{2}} \frac{\alpha}{\pi} \frac{E_{e}}{m_{N}}\left(g_{A} f_{S}\left(E_{e}\right)-g_{A} f_{T}\left(E_{e}\right)-g_{A} g_{S}\left(E_{e}\right)-g_{A} \frac{E_{0}-E_{e}}{E_{e}} h_{S}\left(E_{e}\right)\right. \\
& \left.-\sqrt{1-\beta^{2}} g_{V}\left(E_{e}\right)+g_{A} \sqrt{1-\beta^{2}} h_{A}\left(E_{e}\right)\right) \text {. }
\end{aligned}
$$

Taking into account the contribution of the neutron radiative beta decay $[11,12,14,29,30]$ we obtain the correlation function $\zeta\left(E_{e}\right)_{\mathrm{RC}}$ and correlation coefficients $\zeta\left(E_{e}\right)_{\mathrm{RC}} X\left(E_{e}\right)_{\mathrm{RC}}$ for $X=b, a, A, B, \ldots, T$ and $U$ in Eq. (A17) in the form

$$
\begin{aligned}
\zeta\left(E_{e}\right)_{\mathrm{RC}}= & 1+\frac{\alpha}{\pi} \bar{g}_{n}\left(E_{e}\right)+\frac{1}{1+3 g_{A}^{2}} \frac{\alpha}{\pi} \frac{E_{e}}{m_{N}}\left(f_{V}\left(E_{e}\right)+\sqrt{1-\beta^{2}} f_{S}\left(E_{e}\right)+g_{A} g_{V}\left(E_{e}\right) \beta^{2}+\left(1+2 g_{A}\right) h_{A}\left(E_{e}\right) \beta^{2}\right. \\
& \left.+g_{A} \frac{E_{0}-E_{e}}{E_{e}} \sqrt{1-\beta^{2}} h_{S}\left(E_{e}\right)+3 g_{A}^{2} f_{A}\left(E_{e}\right)+3 g_{A}^{2} \sqrt{1-\beta^{2}} f_{T}\left(E_{e}\right)\right)+\frac{3 g_{A}^{2}}{1+3 g_{A}^{2}} \frac{\alpha}{\pi} \frac{5}{2} \frac{m_{N}^{2}}{M_{W}^{2}} \ell n \frac{M_{W}^{2}}{m_{N}^{2}} \\
& +\frac{1}{1+3 g_{A}^{2}} \frac{\alpha}{\pi}\left(\bar{g}_{\mathrm{st}}\left(E_{e}\right)+3 g_{A} \bar{f}_{\mathrm{st}}\left(E_{e}\right)\right),
\end{aligned}
$$

$\zeta\left(E_{e}\right)_{\mathrm{RC}} b\left(E_{e}\right)_{\mathrm{RC}}=0$, 


$$
\begin{aligned}
& \zeta\left(E_{e}\right)_{\mathrm{RC}} a\left(E_{e}\right)_{\mathrm{RC}}=a_{0}\left(1+\frac{\alpha}{\pi} \bar{g}_{n}\left(E_{e}\right)+\frac{\alpha}{\pi} f_{n}\left(E_{e}\right)\right)+\frac{1}{1+3 g_{A}^{2}} \frac{\alpha}{\pi} \frac{E_{e}}{m_{N}}\left(f_{V}\left(E_{e}\right)+g_{A} \sqrt{1-\beta^{2}} g_{S}\left(E_{e}\right)\right. \\
& \left.+g_{A} g_{V}\left(E_{e}\right)+\left(1-2 g_{A}\right) h_{A}\left(E_{e}\right)-g_{A}^{2} f_{A}\left(E_{e}\right)\right)-\frac{g_{A}^{2}}{1+3 g_{A}^{2}} \frac{\alpha}{\pi} \frac{5}{2} \frac{m_{N}^{2}}{M_{W}^{2}} \ln \frac{M_{W}^{2}}{m_{N}^{2}} \\
& +\frac{1}{1+3 g_{A}^{2}} \frac{\alpha}{\pi}\left(\bar{g}_{\mathrm{st}}\left(E_{e}\right)-g_{A} \bar{f}_{\mathrm{st}}\left(E_{e}\right)\right) \\
& \zeta\left(E_{e}\right)_{\mathrm{RC}} A\left(E_{e}\right)_{\mathrm{RC}}=A_{0}\left(1+\frac{\alpha}{\pi} \bar{g}_{n}\left(E_{e}\right)+\frac{\alpha}{\pi} f_{n}\left(E_{e}\right)\right)+\frac{1}{1+3 g_{A}^{2}} \frac{\alpha}{\pi} \frac{E_{e}}{m_{N}}\left(g_{A} f_{V}\left(E_{e}\right)+\sqrt{1-\beta^{2}} g_{S}\left(E_{e}\right)\right. \\
& \left.+g_{V}\left(E_{e}\right)-g_{A} h_{A}\left(E_{e}\right)+g_{A}\left(1-2 g_{A}\right) f_{A}\left(E_{e}\right)\right)+\frac{1}{1+3 g_{A}^{2}} g_{A}\left(1-2 g_{A}\right) \frac{\alpha}{\pi} \frac{5}{2} \frac{m_{N}^{2}}{M_{W}^{2}} \ln \frac{M_{W}^{2}}{m_{N}^{2}} \\
& +\frac{1}{1+3 g_{A}^{2}} \frac{\alpha}{\pi}\left(g_{A} \bar{g}_{\mathrm{st}}\left(E_{e}\right)+\left(1-2 g_{A}\right) \bar{f}_{\mathrm{st}}\left(E_{e}\right)\right), \\
& \zeta\left(E_{e}\right)_{\mathrm{RC}} B\left(E_{e}\right)_{\mathrm{RC}}=B_{0}\left(1+\frac{\alpha}{\pi} \bar{g}_{n}\left(E_{e}\right)\right)+\frac{1}{1+3 g_{A}^{2}} \frac{\alpha}{\pi} \frac{E_{e}}{m_{N}}\left(g_{A} f_{V}\left(E_{e}\right)+g_{A} \sqrt{1-\beta^{2}} f_{S}\left(E_{e}\right)+\frac{E_{0}-E_{e}}{E_{e}}\right. \\
& \times \sqrt{1-\beta^{2}} h_{S}\left(E_{e}\right)+g_{A} g_{V}\left(E_{e}\right) \beta^{2}-\left(1-2 g_{A}\right) h_{A}\left(E_{e}\right) \beta^{2}+g_{A}\left(1+2 g_{A}\right) f_{A}\left(E_{e}\right)+g_{A}\left(1+2 g_{A}\right) \sqrt{1-\beta^{2}} \\
& \left.\times f_{T}\left(E_{e}\right)\right)+\frac{1}{1+3 g_{A}^{2}} g_{A}\left(1+2 g_{A}\right) \frac{\alpha}{\pi} \frac{5}{2} \frac{m_{N}^{2}}{M_{W}^{2}} \ln \frac{M_{W}^{2}}{m_{N}^{2}}+\frac{1}{1+3 g_{A}^{2}} \frac{\alpha}{\pi}\left(g_{A} \bar{g}_{\mathrm{st}}\left(E_{e}\right)+\left(1+2 g_{A}\right) \bar{f}_{\mathrm{st}}\left(E_{e}\right)\right), \\
& \zeta\left(E_{e}\right)_{\mathrm{RC}} K_{n}\left(E_{e}\right)_{\mathrm{RC}}=\frac{1}{1+3 g_{A}^{2}} \frac{\alpha}{\pi} \frac{E_{e}}{m_{N}}\left(\left(1-g_{A}\right) g_{V}\left(E_{e}\right)+\left(1+2 g_{A}\right) h_{A}\left(E_{e}\right)\right) \\
& \zeta\left(E_{e}\right)_{\mathrm{RC}} Q_{n}\left(E_{e}\right)_{\mathrm{RC}}=0, \\
& \zeta\left(E_{e}\right)_{\mathrm{RC}} G\left(E_{e}\right)_{\mathrm{RC}}=-\left(1+\frac{\alpha}{\pi} \bar{g}_{n}\left(E_{e}\right)+\frac{\alpha}{\pi} f_{n}\left(E_{e}\right)\right)-\frac{1}{1+3 g_{A}^{2}} \frac{\alpha}{\pi} \frac{E_{e}}{m_{N}}\left(f_{V}\left(E_{e}\right)+g_{A} \sqrt{1-\beta^{2}} g_{S}\left(E_{e}\right)\right. \\
& \left.+g_{A} g_{V}\left(E_{e}\right)+\left(1+2 g_{A}\right) h_{A}\left(E_{e}\right)+3 g_{A}^{2} f_{A}\left(E_{e}\right)\right)-\frac{3 g_{A}^{2}}{1+3 g_{A}^{2}} \frac{\alpha}{\pi} \frac{5}{2} \frac{m_{N}^{2}}{M_{W}^{2}} \ln \frac{M_{W}^{2}}{m_{N}^{2}} \\
& -\frac{1}{1+3 g_{A}^{2}} \frac{\alpha}{\pi}\left(\bar{g}_{\mathrm{st}}\left(E_{e}\right)+3 g_{A} \bar{f}_{\mathrm{st}}\left(E_{e}\right)\right) \\
& \zeta\left(E_{e}\right)_{\mathrm{RC}} H\left(E_{e}\right)_{\mathrm{RC}}=-\frac{m_{e}}{E_{e}} a_{0}\left(1+\frac{\alpha}{\pi} \bar{g}_{n}\left(E_{e}\right)+\frac{\alpha}{\pi} h_{n}^{(1)}\left(E_{e}\right)\right)-\frac{1}{1+3 g_{A}^{2}} \frac{\alpha}{\pi} \frac{E_{e}}{m_{N}}\left(\sqrt{1-\beta^{2}} f_{V}\left(E_{e}\right)+f_{S}\left(E_{e}\right)\right. \\
& \left.+g_{A} g_{S}\left(E_{e}\right) \beta^{2}+g_{A} \frac{E_{0}-E_{e}}{E_{e}} h_{S}\left(E_{e}\right)-g_{A}^{2} \sqrt{1-\beta^{2}} f_{A}\left(E_{e}\right)-g_{A}^{2} f_{T}\left(E_{e}\right)\right)+\sqrt{1-\beta^{2}} \frac{g_{A}^{2}}{1+3 g_{A}^{2}} \frac{\alpha}{\pi} \frac{5}{2} \frac{m_{N}^{2}}{M_{W}^{2}} \\
& \times \ln \frac{M_{W}^{2}}{m_{N}^{2}}-\frac{1}{1+3 g_{A}^{2}} \frac{\alpha}{\pi} \sqrt{1-\beta^{2}}\left(\bar{g}_{\mathrm{st}}\left(E_{e}\right)-g_{A} \bar{f}_{\mathrm{st}}\left(E_{e}\right)\right) \\
& \zeta\left(E_{e}\right)_{\mathrm{RC}} N\left(E_{e}\right)_{\mathrm{RC}}=-\frac{m_{e}}{E_{e}} A_{0}\left(1+\frac{\alpha}{\pi} \bar{g}_{n}\left(E_{e}\right)+\frac{\alpha}{\pi} h_{n}^{(1)}\left(E_{e}\right)\right)-\frac{1}{1+3 g_{A}^{2}} \frac{\alpha}{\pi} \frac{E_{e}}{m_{N}}\left(g_{A} f_{V}\left(E_{e}\right) \sqrt{1-\beta^{2}}+g_{A} f_{S}\left(E_{e}\right)\right. \\
& \left.-g_{A} g_{S}\left(E_{e}\right) \beta^{2}+\frac{1}{3}\left(1-2 g_{A}\right) \frac{E_{0}-E_{e}}{E_{e}} h_{S}\left(E_{e}\right)+g_{A}\left(1-2 g_{A}\right) \sqrt{1-\beta^{2}} f_{A}\left(E_{e}\right)+g_{A}\left(1-2 g_{A}\right) f_{T}\left(E_{e}\right)\right) \\
& -\sqrt{1-\beta^{2}} \frac{1}{1+3 g_{A}^{2}} g_{A}\left(1-2 g_{A}\right) \frac{\alpha}{\pi} \frac{5}{2} \frac{m_{N}^{2}}{M_{W}^{2}} \ln \frac{M_{W}^{2}}{m_{N}^{2}}-\frac{1}{1+3 g_{A}^{2}} \frac{\alpha}{\pi} \sqrt{1-\beta^{2}}\left(g_{A} \bar{g}_{\mathrm{st}}\left(E_{e}\right)+\left(1-2 g_{A}\right) \bar{f}_{\mathrm{st}}\left(E_{e}\right)\right),
\end{aligned}
$$




$$
\begin{aligned}
& \zeta\left(E_{e}\right)_{\mathrm{RC}} Q_{e}\left(E_{e}\right)_{\mathrm{RC}}=-A_{0}\left(1+\frac{\alpha}{\pi} \bar{g}_{n}\left(E_{e}\right)+\frac{\alpha}{\pi} h_{n}^{(2)}\left(E_{e}\right)\right)-\frac{1}{1+3 g_{A}^{2}} \frac{\alpha}{\pi} \frac{E_{e}}{m_{N}}\left(g_{A} f_{V}\left(E_{e}\right)-g_{A} f_{S}\left(E_{e}\right)\right. \\
& -g_{A}\left(1+\sqrt{1-\beta^{2}} g_{S}\left(E_{e}\right)+\left(1+\sqrt{1-\beta^{2}}\right) g_{V}\left(E_{e}\right)-\frac{1}{3}\left(1-2 g_{A}\right) \frac{E_{0}-E_{e}}{E_{e}} h_{S}\left(E_{e}\right)+g_{A}\left(1-2 g_{A}\right) f_{A}\left(E_{e}\right)\right. \\
& \left.-g_{A}\left(1-2 g_{A}\right) f_{T}\left(E_{e}\right)\right)-\frac{1}{1+3 g_{A}^{2}} g_{A}\left(1-2 g_{A}\right) \frac{\alpha 5}{\pi} \frac{m_{N}^{2}}{M_{W}^{2}} \ell n \frac{M_{W}^{2}}{m_{N}^{2}}-\frac{1}{1+3 g_{A}^{2}} \frac{\alpha}{\pi}\left(g_{A} \bar{g}_{\mathrm{st}}\left(E_{e}\right)+\left(1-2 g_{A}\right) \bar{f}_{\mathrm{st}}\left(E_{e}\right)\right) \text {, } \\
& \zeta\left(E_{e}\right)_{\mathrm{RC}} K_{e}\left(E_{e}\right)_{\mathrm{RC}}=-a_{0}\left(1+\frac{\alpha}{\pi} \bar{g}_{n}\left(E_{e}\right)+\frac{\alpha}{\pi} h_{n}^{(2)}\left(E_{e}\right)\right)-\frac{1}{1+3 g_{A}^{2}} \frac{\alpha}{\pi} \frac{E_{e}}{m_{N}}\left(f_{V}\left(E_{e}\right)-f_{S}\left(E_{e}\right)\right. \\
& +g_{A}\left(1+\sqrt{1-\beta^{2}}\right) g_{S}\left(E_{e}\right)+g_{A}\left(1+\sqrt{1-\beta^{2}}\right) g_{V}\left(E_{e}\right)+\left(1-2 g_{A}\right)\left(1+\sqrt{1-\beta^{2}}\right) h_{A}\left(E_{e}\right) \\
& \left.-g_{A} \frac{E_{0}-E_{e}}{E_{e}} h_{S}\left(E_{e}\right)-g_{A}^{2} f_{A}\left(E_{e}\right)+g_{A}^{2} f_{T}\left(E_{e}\right)\right)+\frac{g_{A}^{2}}{1+3 g_{A}^{2}} \frac{\alpha}{\pi} \frac{5}{2} \frac{m_{N}^{2}}{M_{W}^{2}} \ell n \frac{M_{W}^{2}}{m_{N}^{2}} \\
& -\frac{1}{1+3 g_{A}^{2}} \frac{\alpha}{\pi}\left(\bar{g}_{\mathrm{st}}\left(E_{e}\right)-g_{A} \bar{f}_{\mathrm{st}}\left(E_{e}\right)\right) \\
& \zeta\left(E_{e}\right)_{\mathrm{RC}} S\left(E_{e}\right)_{\mathrm{RC}}=-\frac{1}{1+3 g_{A}^{2}} \frac{\alpha}{\pi} \frac{E_{e}}{m_{N}}\left(g_{A} f_{S}\left(E_{e}\right)-g_{A} f_{T}\left(E_{e}\right)+g_{A} g_{S}\left(E_{e}\right)-g_{A} \frac{E_{0}-E_{e}}{E_{e}} h_{S}\left(E_{e}\right)\right. \\
& \left.-g_{A} \sqrt{1-\beta^{2}} g_{V}\left(E_{e}\right)+\left(1+2 g_{A}\right) \sqrt{1-\beta^{2}} h_{A}\left(E_{e}\right)\right) \\
& \zeta\left(E_{e}\right)_{\mathrm{RC}} T\left(E_{e}\right)_{\mathrm{RC}}=-B_{0}\left(1+\frac{\alpha}{\pi} \bar{g}_{n}\left(E_{e}\right)+\frac{\alpha}{\pi} f_{n}\left(E_{e}\right)\right)-\frac{1}{1+3 g_{A}^{2}} \frac{\alpha}{\pi} \frac{E_{e}}{m_{N}}\left(g_{A} f_{V}\left(E_{e}\right)-g_{A} g_{S}\left(E_{e}\right)\right. \\
& \left.+g_{A} g_{V}\left(E_{e}\right)-\left(1-2 g_{A}\right) h_{A}\left(E_{e}\right)+g_{A}\left(1+2 g_{A}\right) f_{A}\left(E_{e}\right)\right)-\frac{1}{1+3 g_{A}^{2}} g_{A}\left(1+2 g_{A}\right) \frac{\alpha 5}{\pi} \frac{m_{N}^{2}}{M_{W}^{2}} \ell n \frac{M_{W}^{2}}{m_{N}^{2}} \\
& -\frac{1}{1+3 g_{A}^{2}} \frac{\alpha}{\pi}\left(g_{A} \bar{g}_{\mathrm{st}}\left(E_{e}\right)+\left(1+2 g_{A}\right) \bar{f}_{\mathrm{st}}\left(E_{e}\right)\right) \\
& \zeta\left(E_{e}\right)_{\mathrm{RC}} U\left(E_{e}\right)_{\mathrm{RC}}=\frac{1}{1+3 g_{A}^{2}} \frac{\alpha}{\pi} \frac{E_{e}}{m_{N}}\left(g_{A} f_{S}\left(E_{e}\right)-g_{A} f_{T}\left(E_{e}\right)-g_{A} g_{S}\left(E_{e}\right)-g_{A} \frac{E_{0}-E_{e}}{E_{e}} h_{S}\left(E_{e}\right)\right. \\
& \left.-\sqrt{1-\beta^{2}} g_{V}\left(E_{e}\right)+g_{A} \sqrt{1-\beta^{2}} h_{A}\left(E_{e}\right)\right) \text {. }
\end{aligned}
$$

The functions $\bar{g}_{n}\left(E_{e}\right)$ and $f_{n}\left(E_{e}\right)$ have been calculated by Sirlin [19] and Shann [21] (see also [11,12,29]), whereas the function $h_{n}^{(1)}\left(E_{e}\right)$ and $h_{n}^{(2)}\left(E_{e}\right)$ have been calculated in [12,14]. They are equal to

$$
\begin{aligned}
\bar{g}_{n}\left(E_{e}\right)= & \frac{3}{4} \ell n\left(\frac{m_{N}^{2}}{m_{e}^{2}}\right)-\frac{3}{8}+\left[\frac{1}{\beta} \ell n\left(\frac{1+\beta}{1-\beta}\right)-2\right]\left[\ln \left(\frac{2\left(E_{0}-E_{e}\right)}{m_{e}}\right)-\frac{3}{2}+\frac{1}{3} \frac{E_{0}-E_{e}}{E_{e}}\right]-\frac{2}{\beta} \operatorname{Li}_{2}\left(\frac{2 \beta}{1+\beta}\right) \\
& +\frac{1}{2 \beta} \ell n\left(\frac{1+\beta}{1-\beta}\right)\left[\left(1+\beta^{2}\right)+\frac{1}{12} \frac{\left(E_{0}-E_{e}\right)^{2}}{E_{e}^{2}}-\ell n\left(\frac{1+\beta}{1-\beta}\right)\right] \\
f_{n}\left(E_{e}\right)= & \frac{1}{3} \frac{E_{0}-E_{e}}{E_{e}}\left(1+\frac{1}{8} \frac{E_{0}-E_{e}}{E_{e}}\right) \frac{1-\beta^{2}}{\beta^{2}}\left[\frac{1}{\beta} \ell n\left(\frac{1+\beta}{1-\beta}\right)-2\right]-\frac{1}{12} \frac{\left(E_{0}-E_{e}\right)^{2}}{E_{e}^{2}}+\frac{1-\beta^{2}}{2 \beta} \ell n\left(\frac{1+\beta}{1-\beta}\right), \\
h_{n}^{(1)}\left(E_{e}\right)= & -\frac{1}{3} \frac{E_{0}-E_{e}}{E_{e}}\left\{\left(1+\frac{1+\beta^{2}}{8 \beta^{2}} \frac{E_{0}-E_{e}}{E_{e}}\right)\left[\frac{1}{\beta} \ln \left(\frac{1+\beta}{1-\beta}\right)-2\right]+\frac{1}{4} \frac{E_{0}-E_{e}}{E_{e}}\right\}-\frac{\beta}{2} \ell n\left(\frac{1+\beta}{1-\beta}\right), \\
h_{n}^{(2)}\left(E_{e}\right)= & -\frac{1}{3} \frac{E_{0}-E_{e}}{E_{e}}\left\{\left(1+\frac{1+\beta^{2}}{8 \beta^{2}} \frac{E_{0}-E_{e}}{E_{e}}\right)\left[\frac{1}{\beta} \ell n\left(\frac{1+\beta}{1-\beta}\right)-2\right]+\frac{1}{4} \frac{E_{0}-E_{e}}{E_{e}}\right\}+\left(1+\sqrt{1-\beta^{2}}\right) \\
& \times\left\{\frac{1}{3} \frac{E_{0}-E_{e}}{\beta^{2} E_{e}}\left[\frac{1}{\beta} \ell n\left(\frac{1+\beta}{1-\beta}\right)-2\right]+\frac{1}{24} \frac{\left(E_{0}-E_{e}\right)^{2}}{\beta^{2} E_{e}^{2}}\left(\frac{3-\beta^{2}}{\beta^{2}}\left[\frac{1}{\beta} \ln \left(\frac{1+\beta}{1-\beta}\right)-2\right]-2\right)+\frac{\sqrt{1-\beta^{2}}}{2 \beta} \ell n\left(\frac{1+\beta}{1-\beta}\right)\right\} .
\end{aligned}
$$


The radiative corrections of order $O(\alpha / \pi)$ and $O\left(\alpha E_{e} / m_{N}\right)$ to the neutron lifetime and the correlation coefficients of the electron-energy and angular distribution Eq. (1) are given by

$$
\begin{aligned}
& \zeta\left(E_{e}\right)_{\mathrm{RC}}=1+\frac{\alpha}{\pi} \bar{g}_{n}\left(E_{e}\right)+\zeta\left(E_{e}\right)_{\mathrm{RC}-\mathrm{NLO}} \\
& \zeta\left(E_{e}\right)_{\mathrm{RC}-\mathrm{NLO}}=\frac{1}{1+3 g_{A}^{2}} \frac{\alpha}{\pi} \frac{E_{e}}{m_{N}}\left(f_{V}\left(E_{e}\right)+\sqrt{1-\beta^{2}} f_{S}\left(E_{e}\right)+g_{A} g_{V}\left(E_{e}\right) \beta^{2}+\left(1+2 g_{A}\right) h_{A}\left(E_{e}\right) \beta^{2}\right. \\
& \left.+g_{A} \frac{E_{0}-E_{e}}{E_{e}} \sqrt{1-\beta^{2}} h_{S}\left(E_{e}\right)+3 g_{A}^{2} f_{A}\left(E_{e}\right)+3 g_{A}^{2} \sqrt{1-\beta^{2}} f_{T}\left(E_{e}\right)\right)+\frac{3 g_{A}^{2}}{1+3 g_{A}^{2}} \frac{\alpha}{\pi} \frac{5}{2} \frac{m_{N}^{2}}{M_{W}^{2}} \ln \frac{M_{W}^{2}}{m_{N}^{2}} \\
& +\frac{1}{1+3 g_{A}^{2}} \frac{\alpha}{\pi}\left(\bar{g}_{\mathrm{st}}\left(E_{e}\right)+3 g_{A} \bar{f}_{\mathrm{st}}\left(E_{e}\right)\right) \\
& a\left(E_{e}\right)_{\mathrm{RC}}=a_{0}\left(1+\frac{\alpha}{\pi} f_{n}\left(E_{e}\right)\right)+a\left(E_{e}\right)_{\mathrm{RC}-\mathrm{NLO}}, \\
& a\left(E_{e}\right)_{\mathrm{RC}-\mathrm{NLO}}=\frac{1}{1+3 g_{A}^{2}} \frac{\alpha}{\pi} \frac{E_{e}}{m_{N}}\left(f_{V}\left(E_{e}\right)+g_{A} \sqrt{1-\beta^{2}} g_{S}\left(E_{e}\right)+g_{A} g_{V}\left(E_{e}\right)+\left(1-2 g_{A}\right) h_{A}\left(E_{e}\right)\right. \\
& \left.-g_{A}^{2} f_{A}\left(E_{e}\right)\right)-\frac{g_{A}^{2}}{1+3 g_{A}^{2}} \frac{\alpha}{\pi} \frac{5}{2} \frac{m_{N}^{2}}{M_{W}^{2}} \ell n \frac{M_{W}^{2}}{m_{N}^{2}}+\frac{1}{1+3 g_{A}^{2}} \frac{\alpha}{\pi}\left(\bar{g}_{\mathrm{st}}\left(E_{e}\right)-g_{A} \bar{f}_{\mathrm{st}}\left(E_{e}\right)\right)-a_{0} \zeta\left(E_{e}\right)_{\mathrm{RC}-\mathrm{NLO}}, \\
& A\left(E_{e}\right)_{\mathrm{RC}}=A_{0}\left(1+\frac{\alpha}{\pi} f_{n}\left(E_{e}\right)\right)+A\left(E_{e}\right)_{\mathrm{RC}-\mathrm{NLO}}, \\
& A\left(E_{e}\right)_{\mathrm{RC}-\mathrm{NLO}}=\frac{1}{1+3 g_{A}^{2}} \frac{\alpha}{\pi} \frac{E_{e}}{m_{N}}\left(g_{A} f_{V}\left(E_{e}\right)+\sqrt{1-\beta^{2}} g_{S}\left(E_{e}\right)+g_{V}\left(E_{e}\right)-g_{A} h_{A}\left(E_{e}\right)\right. \\
& \left.+g_{A}\left(1-2 g_{A}\right) f_{A}\left(E_{e}\right)\right)+\frac{1}{1+3 g_{A}^{2}} g_{A}\left(1-2 g_{A}\right) \frac{\alpha}{\pi} \frac{5}{2} \frac{m_{N}^{2}}{M_{W}^{2}} \ell n \frac{M_{W}^{2}}{m_{N}^{2}}+\frac{1}{1+3 g_{A}^{2}} \frac{\alpha}{\pi}\left(g_{A} \bar{g}_{\mathrm{st}}\left(E_{e}\right)+\left(1-2 g_{A}\right) \bar{f}_{\mathrm{st}}\left(E_{e}\right)\right) \\
& -A_{0} \zeta\left(E_{e}\right)_{\mathrm{RC}-\mathrm{NLO}} \\
& B\left(E_{e}\right)_{\mathrm{RC}}=B_{0}\left(1+\frac{\alpha}{\pi} \bar{g}_{n}\left(E_{e}\right)\right)+B\left(E_{e}\right)_{\mathrm{RC}-\mathrm{NLO}}, \\
& B\left(E_{e}\right)_{\mathrm{RC}-\mathrm{NLO}}=\frac{1}{1+3 g_{A}^{2}} \frac{\alpha}{\pi} \frac{E_{e}}{m_{N}}\left(g_{A} f_{V}\left(E_{e}\right)+g_{A} \sqrt{1-\beta^{2}} f_{S}\left(E_{e}\right)+\frac{E_{0}-E_{e}}{E_{e}} \sqrt{1-\beta^{2}} h_{S}\left(E_{e}\right)\right. \\
& \left.+g_{A} g_{V}\left(E_{e}\right) \beta^{2}-\left(1-2 g_{A}\right) h_{A}\left(E_{e}\right) \beta^{2}+g_{A}\left(1+2 g_{A}\right) f_{A}\left(E_{e}\right)+g_{A}\left(1+2 g_{A}\right) \sqrt{1-\beta^{2}} f_{T}\left(E_{e}\right)\right) \\
& +\frac{1}{1+3 g_{A}^{2}} g_{A}\left(1+2 g_{A}\right) \frac{\alpha}{\pi} \frac{5}{2} \frac{m_{N}^{2}}{M_{W}^{2}} \ell n \frac{M_{W}^{2}}{m_{N}^{2}}+\frac{1}{1+3 g_{A}^{2}} \frac{\alpha}{\pi}\left(g_{A} \bar{g}_{\mathrm{st}}\left(E_{e}\right)+\left(1+2 g_{A}\right) \bar{f}_{\mathrm{st}}\left(E_{e}\right)\right) \\
& -B_{0} \zeta\left(E_{e}\right)_{\mathrm{RC}-\mathrm{NLO}} \\
& K_{n}\left(E_{e}\right)_{\mathrm{RC}}=K_{n}\left(E_{e}\right)_{\mathrm{RC}-\mathrm{NLO}}=\frac{1}{1+3 g_{A}^{2}} \frac{\alpha}{\pi} \frac{E_{e}}{m_{N}}\left(\left(1-g_{A}\right) g_{V}\left(E_{e}\right)+\left(1+2 g_{A}\right) h_{A}\left(E_{e}\right)\right), \\
& Q_{n}\left(E_{e}\right)_{\mathrm{RC}}=0, \\
& G\left(E_{e}\right)_{\mathrm{RC}}=-\left(1+\frac{\alpha}{\pi} f_{n}\left(E_{e}\right)\right)+G\left(E_{e}\right)_{\mathrm{RC}-\mathrm{NLO}}, \\
& G\left(E_{e}\right)_{\mathrm{RC}-\mathrm{NLO}}=-\frac{1}{1+3 g_{A}^{2}} \frac{\alpha}{\pi} \frac{E_{e}}{m_{N}}\left(g_{A} \sqrt{1-\beta^{2}} g_{S}\left(E_{e}\right)+g_{A}\left(1-\beta^{2}\right) g_{V}\left(E_{e}\right)+\left(1+2 g_{A}\right)\left(1-\beta^{2}\right)\right. \\
& \left.\times h_{A}\left(E_{e}\right)-\sqrt{1-\beta^{2}} f_{S}\left(E_{e}\right)-g_{A} \frac{E_{0}-E_{e}}{E_{e}} \sqrt{1-\beta^{2}} h_{S}\left(E_{e}\right)-3 g_{A}^{2} \sqrt{1-\beta^{2}} f_{T}\left(E_{e}\right)\right),
\end{aligned}
$$




$$
H\left(E_{e}\right)_{\mathrm{RC}}=-\frac{m_{e}}{E_{e}} a_{0}\left(1+\frac{\alpha}{\pi} h_{n}^{(1)}\left(E_{e}\right)\right)+H\left(E_{e}\right)_{\mathrm{RC}-\mathrm{NLO}}
$$

$H\left(E_{e}\right)_{\mathrm{RC}-\mathrm{NLO}}=-\frac{1}{1+3 g_{A}^{2}} \frac{\alpha}{\pi} \frac{E_{e}}{m_{N}}\left(\sqrt{1-\beta^{2}} f_{V}\left(E_{e}\right)+f_{S}\left(E_{e}\right)+g_{A} g_{S}\left(E_{e}\right) \beta^{2}\right.$

$$
\begin{aligned}
& \left.+g_{A} \frac{E_{0}-E_{e}}{E_{e}} h_{S}\left(E_{e}\right)-g_{A}^{2} \sqrt{1-\beta^{2}} f_{A}\left(E_{e}\right)-g_{A}^{2} f_{T}\left(E_{e}\right)\right)+\sqrt{1-\beta^{2}} \frac{g_{A}^{2}}{1+3 g_{A}^{2}} \frac{\alpha}{\pi} \frac{5}{2} \frac{m_{N}^{2}}{M_{W}^{2}} \ell n \frac{M_{W}^{2}}{m_{N}^{2}} \\
& -\frac{1}{1+3 g_{A}^{2}} \frac{\alpha}{\pi} \sqrt{1-\beta^{2}}\left(\bar{g}_{\mathrm{st}}\left(E_{e}\right)-g_{A} \bar{f}_{\mathrm{st}}\left(E_{e}\right)\right)+\frac{m_{e}}{E_{e}} a_{0} \zeta\left(E_{e}\right)_{\mathrm{RC}-\mathrm{NLO}},
\end{aligned}
$$

$$
N\left(E_{e}\right)_{\mathrm{RC}}=-\frac{m_{e}}{E_{e}} A_{0}\left(1+\frac{\alpha}{\pi} h_{n}^{(1)}\left(E_{e}\right)\right)+N\left(E_{e}\right)_{\mathrm{RC}-\mathrm{NLO}}
$$

$$
\begin{aligned}
N\left(E_{e}\right)_{\mathrm{RC}-\mathrm{NLO}}= & -\frac{1}{1+3 g_{A}^{2}} \frac{\alpha}{\pi} \frac{E_{e}}{m_{N}}\left(g_{A} f_{V}\left(E_{e}\right) \sqrt{1-\beta^{2}}+g_{A} f_{S}\left(E_{e}\right)-g_{A} g_{S}\left(E_{e}\right) \beta^{2}\right. \\
& \left.+\frac{1}{3}\left(1-2 g_{A}\right) \frac{E_{0}-E_{e}}{E_{e}} h_{S}\left(E_{e}\right)+g_{A}\left(1-2 g_{A}\right) \sqrt{1-\beta^{2}} f_{A}\left(E_{e}\right)+g_{A}\left(1-2 g_{A}\right) f_{T}\left(E_{e}\right)\right)-\sqrt{1-\beta^{2}} \frac{1}{1+3 g_{A}^{2}} \\
& \times g_{A}\left(1-2 g_{A}\right) \frac{\alpha}{\pi} \frac{m_{N}^{2}}{M_{W}^{2}} \ell n \frac{M_{W}^{2}}{m_{N}^{2}}-\frac{1}{1+3 g_{A}^{2}} \frac{\alpha}{\pi} \sqrt{1-\beta^{2}}\left(g_{A} \bar{g}_{\mathrm{st}}\left(E_{e}\right)+\left(1-2 g_{A}\right) \bar{f}_{\mathrm{st}}\left(E_{e}\right)\right)+\frac{m_{e}}{E_{e}} A_{0} \zeta\left(E_{e}\right)_{\mathrm{RC}-\mathrm{NLO}}, \\
Q_{e}\left(E_{e}\right)_{\mathrm{RC}}= & -A_{0}\left(1+\frac{\alpha}{\pi} h_{n}^{(2)}\left(E_{e}\right)\right)+Q_{e}\left(E_{e}\right)_{\mathrm{RC}-\mathrm{NLO}} \\
Q_{e}\left(E_{e}\right)_{\mathrm{RC}-\mathrm{NLO}}= & -\frac{1}{1+3 g_{A}^{2}} \frac{\alpha}{\pi} \frac{E_{e}}{m_{N}}\left(g_{A} f_{V}\left(E_{e}\right)-g_{A} f_{S}\left(E_{e}\right)-g_{A}\left(1+\sqrt{1-\beta^{2}}\right) g_{S}\left(E_{e}\right)\right.
\end{aligned}
$$

$$
\left.+\left(1+\sqrt{1-\beta^{2}}\right) g_{V}\left(E_{e}\right)-\frac{1}{3}\left(1-2 g_{A}\right) \frac{E_{0}-E_{e}}{E_{e}} h_{S}\left(E_{e}\right)+g_{A}\left(1-2 g_{A}\right) f_{A}\left(E_{e}\right)-g_{A}\left(1-2 g_{A}\right) f_{T}\left(E_{e}\right)\right)
$$$$
-\frac{1}{1+3 g_{A}^{2}} g_{A}\left(1-2 g_{A}\right) \frac{\alpha}{\pi} \frac{5}{2} \frac{m_{N}^{2}}{M_{W}^{2}} \ell n \frac{M_{W}^{2}}{m_{N}^{2}}-\frac{1}{1+3 g_{A}^{2}} \frac{\alpha}{\pi}\left(g_{A} \bar{g}_{\mathrm{st}}\left(E_{e}\right)+\left(1-2 g_{A}\right) \bar{f}_{\mathrm{st}}\left(E_{e}\right)\right)+A_{0} \zeta\left(E_{e}\right)_{\mathrm{RC}-\mathrm{NLO}}
$$$$
K_{e}\left(E_{e}\right)_{\mathrm{RC}}=-a_{0}\left(1+\frac{\alpha}{\pi} h_{n}^{(2)}\left(E_{e}\right)\right)+K_{e}\left(E_{e}\right)_{\mathrm{RC}-\mathrm{NLO}}
$$

$K_{e}\left(E_{e}\right)_{\mathrm{RC}-\mathrm{NLO}}=-\frac{1}{1+3 g_{A}^{2}} \frac{\alpha}{\pi} \frac{E_{e}}{m_{N}}\left(f_{V}\left(E_{e}\right)-f_{S}\left(E_{e}\right)+g_{A}\left(1+\sqrt{1-\beta^{2}}\right) g_{S}\left(E_{e}\right)\right.$

$$
\begin{aligned}
& \left.+g_{A}\left(1+\sqrt{1-\beta^{2}}\right) g_{V}\left(E_{e}\right)+\left(1-2 g_{A}\right)\left(1+\sqrt{1-\beta^{2}}\right) h_{A}\left(E_{e}\right)-g_{A} \frac{E_{0}-E_{e}}{E_{e}} h_{S}\left(E_{e}\right)-g_{A}^{2} f_{A}\left(E_{e}\right)+g_{A}^{2} f_{T}\left(E_{e}\right)\right) \\
& +\frac{g_{A}^{2}}{1+3 g_{A}^{2}} \frac{\alpha}{\pi} \frac{5}{2} \frac{m_{N}^{2}}{M_{W}^{2}} \ell n \frac{M_{W}^{2}}{m_{N}^{2}}-\frac{1}{1+3 g_{A}^{2}} \frac{\alpha}{\pi}\left(\bar{g}_{\mathrm{st}}\left(E_{e}\right)-g_{A} \bar{f}_{\mathrm{st}}\left(E_{e}\right)\right)+a_{0} \zeta\left(E_{e}\right)_{\mathrm{RC}-\mathrm{NLO}} \\
S\left(E_{e}\right)_{\mathrm{RC}}= & S\left(E_{e}\right)_{\mathrm{RC}-\mathrm{NLO}}=-\frac{1}{1+3 g_{A}^{2}} \frac{\alpha}{\pi} \frac{E_{e}}{m_{N}}\left(g_{A} f_{S}\left(E_{e}\right)-g_{A} f_{T}\left(E_{e}\right)+g_{A} g_{S}\left(E_{e}\right)-g_{A} \frac{E_{0}-E_{e}}{E_{e}} h_{S}\left(E_{e}\right)\right.
\end{aligned}
$$

$$
\left.-g_{A} \sqrt{1-\beta^{2}} g_{V}\left(E_{e}\right)+\left(1+2 g_{A}\right) \sqrt{1-\beta^{2}} h_{A}\left(E_{e}\right)\right)
$$

$$
T\left(E_{e}\right)_{\mathrm{RC}}=-B_{0}\left(1+\frac{\alpha}{\pi} f_{n}\left(E_{e}\right)\right)+T\left(E_{e}\right)_{\mathrm{RC}-\mathrm{NLO}},
$$

$T\left(E_{e}\right)_{\mathrm{RC}-\mathrm{NLO}}=-\frac{1}{1+3 g_{A}^{2}} \frac{\alpha}{\pi} \frac{E_{e}}{m_{N}}\left(g_{A} f_{V}\left(E_{e}\right)-g_{A} g_{S}\left(E_{e}\right)+g_{A} g_{V}\left(E_{e}\right)-\left(1-2 g_{A}\right) h_{A}\left(E_{e}\right)\right.$

$$
\begin{aligned}
& \left.+g_{A}\left(1+2 g_{A}\right) f_{A}\left(E_{e}\right)\right)-\frac{1}{1+3 g_{A}^{2}} g_{A}\left(1+2 g_{A}\right) \frac{\alpha}{\pi} \frac{5}{2} \frac{m_{N}^{2}}{M_{W}^{2}} \ell n \frac{M_{W}^{2}}{m_{N}^{2}}-\frac{1}{1+3 g_{A}^{2}} \frac{\alpha}{\pi}\left(g_{A} \bar{g}_{\mathrm{st}}\left(E_{e}\right)+\left(1+2 g_{A}\right) \bar{f}_{\mathrm{st}}\left(E_{e}\right)\right) \\
& +B_{0} \zeta\left(E_{e}\right)_{\mathrm{RC}-\mathrm{NLO}}
\end{aligned}
$$




$$
\begin{aligned}
U\left(E_{e}\right)_{\mathrm{RC}}=U\left(E_{e}\right)_{\mathrm{RC}-\mathrm{NLO}}= & \frac{1}{1+3 g_{A}^{2}} \frac{\alpha}{\pi} \frac{E_{e}}{m_{N}}\left(g_{A} f_{S}\left(E_{e}\right)-g_{A} f_{T}\left(E_{e}\right)-g_{A} g_{S}\left(E_{e}\right)-g_{A} \frac{E_{0}-E_{e}}{E_{e}} h_{S}\left(E_{e}\right)\right. \\
& \left.-\sqrt{1-\beta^{2}} g_{V}\left(E_{e}\right)+g_{A} \sqrt{1-\beta^{2}} h_{A}\left(E_{e}\right)\right)
\end{aligned}
$$

The correlation function $\zeta\left(E_{e}\right)_{\mathrm{RC}}$ and correlation coefficients $X\left(E_{e}\right)_{\mathrm{RC}}$ contain a complete set of outer radiative corrections of order $O(\alpha / \pi)[12,14,19,21]$, calculated to LO in the large nucleon mass $m_{N}$ expansion, and radiative corrections of order $O\left(\alpha E_{e} / m_{N}\right)[16,17]$, obtained as NLO corrections in the large nucleon mass $m_{N}$ expansion to Sirlin's outer and inner radiative corrections, calculated to LO in the large nucleon mass $m_{N}$ expansion. For $\alpha=0$ the correlation function $\zeta\left(E_{e}\right)_{\mathrm{RC}}$ and the correlation coefficients $X\left(E_{e}\right)_{\mathrm{RC}}$ acquire their expressions, calculated to LO in the large nucleon mass $m_{N}$ expansion $[1,2]$ (see also [29]). We have plotted the $Y\left(E_{e}\right)_{\mathrm{RC}-\mathrm{NLO}}$ corrections for $Y=\zeta, a, A, B, \ldots, U$ and $A^{(\beta)}\left(E_{e}\right)$ (see Appendix E) in $[46]$.

\section{APPENDIX B: THE CORRECTIONS OF ORDER $O\left(E_{e} / m_{N}\right)$ AND $O\left(E_{e}^{2} / m_{N}^{2}\right)$, CAUSED BY WEAK MAGNETISM AND PROTON RECOIL, TO NEXT-TO-LEADING AND NEXT-TO-NEXT-TO-LEADING ORDER IN THE LARGE NUCLEON MASS $m_{N}$ EXPANSION}

The corrections to the structure function $\zeta\left(E_{e}\right)_{\mathrm{WP}}$ and the correlation coefficients $X\left(E_{e}\right)_{\mathrm{WP}}$ for $X=a, A, B, \ldots, T$ and $U$, caused by weak magnetism and proton recoil, we define as follows:

$$
\begin{gathered}
\zeta\left(E_{e}\right)_{\mathrm{WP}}=\zeta\left(E_{e}\right)_{\mathrm{NLO}}+\zeta\left(E_{e}\right)_{\mathrm{N}^{2} \mathrm{LO}}, \\
X\left(E_{e}\right)_{\mathrm{WP}}=X\left(E_{e}\right)_{\mathrm{NLO}}+X\left(E_{e}\right)_{\mathrm{N}^{2} \mathrm{LO}}
\end{gathered}
$$

The corrections $\zeta\left(E_{e}\right)_{\mathrm{NLO}}$ and $X\left(E_{e}\right)_{\mathrm{NLO}}$, which are in principle of order $10^{-3}[11,12,14]$ have been calculated in [47] and $[11,12,14,15,22,23,29,30]$ (see also [24]). The analytical expressions of these corrections are given by

$$
\begin{aligned}
\zeta\left(E_{e}\right)_{\mathrm{NLO}}= & \frac{1}{1+3 g_{A}^{2}} \frac{E_{0}}{m_{N}}\left[-2 g_{A}\left(g_{A}+(\kappa+1)\right)+\left(10 g_{A}^{2}+4(\kappa+1) g_{A}+2\right) \frac{E_{e}}{E_{0}}-2 g_{A}\left(g_{A}+(\kappa+1)\right) \frac{m_{e}^{2}}{E_{0}^{2}} \frac{E_{0}}{E_{e}}\right], \\
a\left(E_{e}\right)_{\mathrm{NLO}}= & \frac{1}{1+3 g_{A}^{2}} \frac{E_{0}}{m_{N}}\left[2 g_{A}\left(g_{A}+(\kappa+1)\right)-4 g_{A}\left(3 g_{A}+(\kappa+1)\right) \frac{E_{e}}{E_{0}}\right]-a_{0} \zeta\left(E_{e}\right)_{\mathrm{NLO}}, \\
A\left(E_{e}\right)_{\mathrm{NLO}}= & \frac{1}{1+3 g_{A}^{2}} \frac{E_{0}}{m_{N}}\left[\left(g_{A}^{2}+\kappa g_{A}-(\kappa+1)\right)-\left(5 g_{A}^{2}+(3 \kappa-4) g_{A}-(\kappa+1)\right) \frac{E_{e}}{E_{0}}\right]-A_{0} \zeta\left(E_{e}\right)_{\mathrm{NLO}}, \\
B\left(E_{e}\right)_{\mathrm{NLO}}= & \frac{1}{1+3 g_{A}^{2}} \frac{E_{0}}{m_{N}}\left[-2 g_{A}\left(g_{A}+(\kappa+1)\right)+\left(7 g_{A}^{2}+(3 \kappa+8) g_{A}+(\kappa+1)\right) \frac{E_{e}}{E_{0}}\right. \\
& \left.-\left(g_{A}^{2}+(\kappa+2) g_{A}+(\kappa+1)\right) \frac{m_{e}^{2}}{E_{0}^{2}} \frac{E_{0}}{E_{e}}\right]-B_{0} \zeta\left(E_{e}\right)_{\mathrm{NLO}}, \\
K_{n}\left(E_{e}\right)_{\mathrm{NLO}}= & \frac{1}{1+3 g_{A}^{2}} \frac{E_{0}}{m_{N}}\left(5 g_{A}^{2}+(\kappa-4) g_{A}-(\kappa+1)\right) \frac{E_{e}}{E_{0}}, \\
Q_{n}\left(E_{e}\right)_{\mathrm{NLO}}= & \frac{1}{1+3 g_{A}^{2}} \frac{E_{0}}{m_{N}}\left[\left(g_{A}^{2}+(\kappa+2) g_{A}+(\kappa+1)\right)-\left(7 g_{A}^{2}+(\kappa+8) g_{A}+(\kappa+1)\right) \frac{E_{e}}{E_{0}}\right], \\
G\left(E_{e}\right)_{\mathrm{NLO}}= & \frac{1}{1+3 g_{A}^{2}} \frac{E_{0}}{m_{N}}\left[-2 g_{A}\left(g_{A}+(\kappa+1)\right) \frac{m_{e}^{2}}{E_{0}^{2}} \frac{E_{0}}{E_{e}}\right], \\
H\left(E_{e}\right)_{\mathrm{NLO}}= & \frac{1}{1+3 g_{A}^{2}} \frac{E_{0}}{m_{N}} \frac{m_{e}}{E_{e}}\left[-2 g_{A}\left(g_{A}+(\kappa+1)\right)+\left(4 g_{A}^{2}+2(\kappa+1) g_{A}-2\right) \frac{E_{e}}{E_{0}}\right]+a_{0} \frac{m_{e}}{E_{e}} \zeta\left(E_{e}\right)_{\mathrm{NLO}},
\end{aligned}
$$




$$
\begin{aligned}
N\left(E_{e}\right)_{\mathrm{NLO}}= & \frac{1}{1+3 g_{A}^{2}} \frac{E_{0}}{m_{N}} \frac{m_{e}}{E_{e}}\left[-\left(\frac{4}{3} g_{A}^{2}+\left(\frac{4}{3} \kappa-\frac{1}{3}\right) g_{A}-\frac{2}{3}(\kappa+1)\right)\right. \\
& \left.+\left(\frac{16}{3} g_{A}^{2}+\left(\frac{4}{3} \kappa-\frac{16}{3}\right) g_{A}-\frac{2}{3}(\kappa+1)\right) \frac{E_{e}}{E_{0}}\right]+A_{0} \frac{m_{e}}{E_{e}} \zeta\left(E_{e}\right)_{\mathrm{NLO}} \\
Q_{e}\left(E_{e}\right)_{\mathrm{NLO}}= & \frac{1}{1+3 g_{A}^{2}} \frac{E_{0}}{m_{N}}\left[-\left(\frac{4}{3} g_{A}^{2}+\left(\frac{4}{3} \kappa-\frac{1}{3}\right) g_{A}-\frac{2}{3}(\kappa+1)\right)+2 g_{A}\left(g_{A}+(\kappa+1)\right) \frac{m_{e}}{E_{0}}\right. \\
& \left.+\left(\frac{22}{3} g_{A}^{2}+\left(\frac{10}{3} \kappa-\frac{10}{3}\right) g_{A}-\frac{2}{3}(\kappa+1)\right) \frac{E_{e}}{E_{0}}\right]+A_{0} \zeta\left(E_{e}\right)_{\mathrm{NLO}}, \\
K_{e}\left(E_{e}\right)_{\mathrm{NLO}}= & \frac{1}{1+3 g_{A}^{2}} \frac{E_{0}}{m_{N}}\left[-2 g_{A}\left(g_{A}+(\kappa+1)\right)+\left(8 g_{A}^{2}+2(\kappa+1) g_{A}+2\right) \frac{m_{e}}{E_{0}}+4 g_{A}\left(3 g_{A}+(\kappa+1)\right) \frac{E_{e}}{E_{0}}\right]+a_{0} \zeta\left(E_{e}\right)_{\mathrm{NLO}}, \\
S\left(E_{e}\right)_{\mathrm{NLO}}= & \frac{1}{1+3 g_{A}^{2}} \frac{m_{e}}{m_{N}}\left(-5 g_{A}^{2}-(\kappa-4) g_{A}+(\kappa+1)\right), \\
T\left(E_{e}\right)_{\mathrm{NLO}}= & \frac{1}{1+3 g_{A}^{2}} \frac{E_{0}}{m_{N}}\left[2 g_{A}\left(g_{A}+(\kappa+1)\right)-\left(7 g_{A}^{2}+(3 \kappa+8) g_{A}+(\kappa+1)\right) \frac{E_{e}}{E_{0}}\right]+B_{0} \zeta\left(E_{e}\right)_{\mathrm{NLO}}, \\
U\left(E_{e}\right)_{\mathrm{NLO}}= & 0 .
\end{aligned}
$$

For the definition of the $O\left(E_{e}^{2} / m_{N}^{2}\right)$ corrections it is convenient to adduce the following expressions, calculated in $[11,12,14]$ :

$$
\begin{aligned}
\bar{a}\left(E_{e}\right)_{\mathrm{NLO}}= & \frac{1}{1+3 g_{A}^{2}} \frac{E_{0}}{m_{N}}\left[2 g_{A}\left(g_{A}+(\kappa+1)\right)-4 g_{A}\left(3 g_{A}+(\kappa+1)\right) \frac{E_{e}}{E_{0}}\right] \\
\bar{A}\left(E_{e}\right)_{\mathrm{NLO}}= & \frac{1}{1+3 g_{A}^{2}} \frac{E_{0}}{m_{N}}\left[\left(g_{A}^{2}+\kappa g_{A}-(\kappa+1)\right)-\left(5 g_{A}^{2}+(3 \kappa-4) g_{A}-(\kappa+1)\right) \frac{E_{e}}{E_{0}}\right] \\
\bar{B}\left(E_{e}\right)_{\mathrm{NLO}}= & \frac{1}{1+3 g_{A}^{2}} \frac{E_{0}}{m_{N}}\left[-2 g_{A}\left(g_{A}+(\kappa+1)\right)+\left(7 g_{A}^{2}+(3 \kappa+8) g_{A}+(\kappa+1)\right) \frac{E_{e}}{E_{0}}-\left(g_{A}^{2}+(\kappa+2) g_{A}+(\kappa+1)\right) \frac{m_{e}^{2}}{E_{0}^{2}} \frac{E_{0}}{E_{e}}\right], \\
\bar{K}_{n}\left(E_{e}\right)_{\mathrm{NLO}}= & \frac{1}{1+3 g_{A}^{2}} \frac{E_{0}}{m_{N}}\left(5 g_{A}^{2}+(\kappa-4) g_{A}-(\kappa+1)\right) \frac{E_{e}}{E_{0}} \\
\bar{Q}_{n}\left(E_{e}\right)_{\mathrm{NLO}}= & \frac{1}{1+3 g_{A}^{2}} \frac{E_{0}}{m_{N}}\left[\left(g_{A}^{2}+(\kappa+2) g_{A}+(\kappa+1)\right)-\left(7 g_{A}^{2}+(\kappa+8) g_{A}+(\kappa+1)\right) \frac{E_{e}}{E_{0}}\right] \\
\bar{G}\left(E_{e}\right)_{\mathrm{NLO}}= & \frac{1}{1+3 g_{A}^{2}} \frac{E_{0}}{m_{N}}\left[\left(2 g_{A}^{2}+2(\kappa+1) g_{A}\right)-\left(10 g_{A}^{2}+4(\kappa+1) g_{A}+2\right) \frac{E_{e}}{E_{0}}\right] \\
\bar{H}\left(E_{e}\right)_{\mathrm{NLO}}= & \frac{1}{1+3 g_{A}^{2}} \frac{E_{0}}{m_{N}} \frac{m_{e}}{E_{e}}\left[-2 g_{A}\left(g_{A}+(\kappa+1)\right)+\left(4 g_{A}^{2}+2(\kappa+1) g_{A}-2\right) \frac{E_{e}}{E_{0}}\right] \\
\bar{N}\left(E_{e}\right)_{\mathrm{NLO}}= & \frac{1}{1+3 g_{A}^{2}} \frac{E_{0}}{m_{N}} \frac{m_{e}}{E_{e}}\left[-\left(\frac{4}{3} g_{A}^{2}+\left(\frac{4}{3} \kappa-\frac{1}{3}\right) g_{A}-\frac{2}{3}(\kappa+1)\right)+\left(\frac{16}{3} g_{A}^{2}+\left(\frac{4}{3} \kappa-\frac{16}{3}\right) g_{A}-\frac{2}{3}(\kappa+1)\right) \frac{E_{e}}{E_{0}}\right] \\
\bar{Q}\left(E_{e}\right)_{\mathrm{NLO}}= & \frac{1}{1+3 g_{A}^{2}} \frac{E_{0}}{m_{N}}\left[-\left(\frac{4}{3} g_{A}^{2}+\left(\frac{4}{3} \kappa-\frac{1}{3}\right) g_{A}-\frac{2}{3}(\kappa+1)\right)+\left(2 g_{A}^{2}+(2 \kappa+1) g_{A}\right) \frac{m_{e}}{E_{0}}\right. \\
& \left.+\left(\frac{22}{3} g_{A}^{2}+\left(\frac{10}{3} \kappa-\frac{10}{3}\right) g_{A}-\frac{2}{3}(\kappa+1)\right) \frac{E_{e}}{E_{0}}\right] \\
\bar{K}\left(E_{e}\right)_{\mathrm{NLO}}= & \frac{1}{1+3 g_{A}^{2}} \frac{E_{0}}{m_{N}}\left[-2 g_{A}\left(g_{A}+(\kappa+1)\right)+\left(8 g_{A}^{2}+2(\kappa+1) g_{A}+2\right) \frac{m_{e}}{E_{0}}+4 g_{A}\left(3 g_{A}+(\kappa+1)\right) \frac{E_{0}}{E_{e}}\right]
\end{aligned}
$$

where $\bar{K}_{n}\left(E_{e}\right)_{\mathrm{NLO}}=K_{n}\left(E_{e}\right)_{\mathrm{NLO}}$ and $\bar{Q}_{n}\left(E_{e}\right)_{\mathrm{NLO}}=Q_{n}\left(E_{e}\right)_{\mathrm{NLO}}$. Using the results obtained in [24], we get the following analytical expressions for the $\mathrm{N}^{2} \mathrm{LO}$ corrections $\zeta\left(E_{e}\right)_{\mathrm{N}^{2} \mathrm{LO}}$ and $X\left(E_{e}\right)_{\mathrm{N}^{2} \mathrm{LO}}$ for $X\left(E_{e}\right)=a\left(E_{e}\right), A\left(E_{e}\right), \ldots, U\left(E_{e}\right)$. They are given by 


$$
\begin{aligned}
& \zeta\left(E_{e}\right)_{\mathrm{N}^{2} \mathrm{LO}}=6 \frac{E_{e}^{2}}{m_{N}^{2}}\left\{\left(1-\frac{1}{4} \frac{E_{0}}{E_{e}}\right)+\frac{1}{3}\left[1-2 a_{0}\left(1-\frac{1}{8} \frac{E_{0}}{E_{e}}\right)\right]\left(1-\frac{m_{e}^{2}}{E_{e}^{2}}\right)\right\}+3 \frac{E_{e}}{m_{N}} \zeta\left(E_{e}\right)_{\mathrm{NLO}}-\frac{E_{e}}{m_{N}} \bar{a}\left(E_{e}\right)_{\mathrm{NLO}} \\
& \times\left(1-\frac{m_{e}^{2}}{E_{e}^{2}}\right)-\frac{8}{1+3 g_{A}^{2}}\left(\frac{E_{0}^{2}}{M_{V}^{2}}+3 g_{A}^{2} \frac{E_{0}^{2}}{M_{A}^{2}}\right) \frac{E_{e}}{E_{0}}\left(1-\frac{E_{e}}{E_{0}}\right), \\
& a\left(E_{e}\right)_{\mathrm{N}^{2} \mathrm{LO}}=12 \frac{E_{e}^{2}}{m_{N}^{2}}\left\{-\left(1-\frac{1}{8} \frac{E_{0}}{E_{e}}\right)+\frac{1}{3} a_{0}\left(1-\frac{1}{4} \frac{E_{0}}{E_{e}}\right)\right\}+3 \frac{E_{e}}{m_{N}} \bar{a}\left(E_{e}\right)_{\mathrm{NLO}}-\frac{E_{e}}{m_{N}} \zeta\left(E_{e}\right)_{\mathrm{NLO}} \\
& -a\left(E_{e}\right)_{\mathrm{NLO}} \zeta\left(E_{e}\right)_{\mathrm{NLO}}+\frac{8}{1+3 g_{A}^{2}}\left(\frac{E_{0}^{2}}{M_{V}^{2}}+3 g_{A}^{2} \frac{E_{0}^{2}}{M_{A}^{2}}\right) \frac{E_{e}}{E_{0}}\left(1-\frac{E_{e}}{E_{0}}\right) \text {, } \\
& A\left(E_{e}\right)_{\mathrm{N}^{2} \mathrm{LO}}=3 \frac{E_{e}}{m_{N}} \bar{A}\left(E_{e}\right)_{\mathrm{NLO}}-\frac{E_{e}}{m_{N}} \bar{K}_{n}\left(E_{e}\right)_{\mathrm{NLO}}\left(1-\frac{m_{e}^{2}}{E_{e}^{2}}\right)-A\left(E_{e}\right)_{\mathrm{NLO}} \zeta\left(E_{e}\right)_{\mathrm{NLO}}, \\
& B\left(E_{e}\right)_{\mathrm{N}^{2} \mathrm{LO}}=6 \frac{E_{e}^{2}}{m_{N}^{2}} B_{0}\left(1-\frac{1}{4} \frac{E_{0}}{E_{e}}\right)+3 \frac{E_{e}}{m_{N}} \bar{B}\left(E_{e}\right)_{\mathrm{NLO}}-B\left(E_{e}\right)_{\mathrm{NLO}} \zeta\left(E_{e}\right)_{\mathrm{NLO}}-B_{0} \zeta\left(E_{e}\right)_{\mathrm{N}^{2} \mathrm{LO}} \\
& -\frac{8 g_{A}}{1+3 g_{A}^{2}}\left(\frac{E_{0}^{2}}{M_{V}^{2}}+\left(1+2 g_{A}\right) \frac{E_{0}^{2}}{M_{A}^{2}}\right) \frac{E_{e}}{E_{0}}\left(1-\frac{E_{e}}{E_{0}}\right) \text {, } \\
& K_{n}\left(E_{e}\right)_{\mathrm{N}^{2} \mathrm{LO}}=3 \frac{E_{e}}{m_{N}} \bar{K}_{n}\left(E_{e}\right)_{\mathrm{NLO}}-3 \frac{E_{e}}{m_{N}} \bar{A}\left(E_{e}\right)_{\mathrm{NLO}}, \\
& Q_{n}\left(E_{e}\right)_{\mathrm{N}^{2} \mathrm{LO}}=-12 \frac{E_{e}^{2}}{m_{N}^{2}} B_{0}\left(1-\frac{1}{8} \frac{E_{0}}{E_{e}}\right)+3 \frac{E_{e}}{m_{N}} \bar{Q}_{n}\left(E_{e}\right)_{\mathrm{NLO}}-3 \frac{E_{e}}{m_{N}} \bar{B}\left(E_{e}\right)_{\mathrm{NLO}}-Q_{n}\left(E_{e}\right)_{\mathrm{NLO}} \zeta\left(E_{e}\right)_{\mathrm{NLO}}, \\
& G\left(E_{e}\right)_{\mathrm{N}^{2} \mathrm{LO}}=6 \frac{E_{e}^{2}}{m_{N}^{2}}\left\{-\left(1-\frac{1}{4} \frac{E_{0}}{E_{e}}\right)-\frac{1}{3}\left(1-\frac{m_{e}^{2}}{E_{e}^{2}}\right)+\frac{2}{3} a_{0}\left(1-\frac{1}{8} \frac{E_{0}}{E_{e}}\right)\right\}+3 \frac{E_{e}}{m_{N}} \bar{G}\left(E_{e}\right)_{\mathrm{NLO}}-\frac{E_{e}}{m_{N}} \bar{H}\left(E_{e}\right)_{\mathrm{NLO}} \\
& -\frac{E_{e}}{m_{N}} \bar{K}_{e}\left(E_{e}\right)_{\mathrm{NLO}}\left(1-\frac{m_{e}}{E_{e}}\right)-G\left(E_{e}\right)_{\mathrm{NLO}} \zeta\left(E_{e}\right)_{\mathrm{NLO}}+\zeta\left(E_{e}\right)_{\mathrm{N}^{2} \mathrm{LO}}+\frac{8}{1+3 g_{A}^{2}}\left(\frac{E_{0}^{2}}{M_{V}^{2}}+3 g_{A}^{2} \frac{E_{0}^{2}}{M_{A}^{2}}\right) \frac{E_{e}}{E_{0}}\left(1-\frac{E_{e}}{E_{0}}\right),
\end{aligned}
$$

$H\left(E_{e}\right)_{\mathrm{N}^{2} \mathrm{LO}}=-\bar{H}\left(E_{e}\right)_{\mathrm{NLO}} \zeta\left(E_{e}\right)_{\mathrm{NLO}}$,

$N\left(E_{e}\right)_{\mathrm{N}^{2} \mathrm{LO}}=-\bar{N}\left(E_{e}\right)_{\mathrm{NLO}} \zeta\left(E_{e}\right)_{\mathrm{NLO}}$,

$Q_{e}\left(E_{e}\right)_{\mathrm{N}^{2} \mathrm{LO}}=3 \frac{E_{e}}{m_{N}} \bar{Q}_{e}\left(E_{e}\right)_{\mathrm{NLO}}-Q_{e}\left(E_{e}\right)_{\mathrm{NLO}} \zeta\left(E_{e}\right)_{\mathrm{NLO}}$,

$K_{e}\left(E_{e}\right)_{\mathrm{N}^{2} \mathrm{LO}}=12 \frac{E_{e}^{2}}{m_{N}^{2}}\left\{\left(1-\frac{1}{8} \frac{E_{0}}{E_{e}}\right)\left(1+\frac{m_{e}}{E_{e}}\right)-\frac{1}{2} a_{0}\left(1-\frac{1}{4} \frac{E_{0}}{E_{e}}\right)\right\}+3 \frac{E_{e}}{m_{N}} \bar{K}_{e}\left(E_{e}\right)_{\mathrm{NLO}}-3 \frac{E_{e}}{m_{N}} \bar{G}\left(E_{e}\right)_{\mathrm{NLO}}$,

$$
\begin{aligned}
\times\left(1+\frac{m_{e}}{E_{e}}\right)-K_{e}\left(E_{e}\right)_{\mathrm{NLO}} \zeta\left(E_{e}\right)_{\mathrm{NLO}}-\frac{8}{1+3 g_{A}^{2}}\left(\frac{E_{0}^{2}}{M_{V}^{2}}+3 g_{A}^{2} \frac{E_{0}^{2}}{M_{A}^{2}}\right) \frac{E_{e}}{E_{0}}\left(1-\frac{E_{e}}{E_{0}}\right), \\
S\left(E_{e}\right)_{\mathrm{N}^{2} \mathrm{LO}}=\frac{1}{1+3 g_{A}^{2}} \frac{E_{0}^{2}}{2 m_{N}^{2}} \frac{m_{e}}{E_{0}}\left\{\left(7 g_{A}^{2}+(\kappa-6) g_{A}+(\kappa+1)\right)+\left(-50 g_{A}^{2}+50 g_{A}-2(\kappa+1)\right) \frac{E_{e}}{E_{0}}\right\}
\end{aligned}
$$$$
-3 \frac{E_{e}}{m_{N}} \bar{N}\left(E_{e}\right)_{\mathrm{NLO}}-S\left(E_{e}\right)_{\mathrm{NLO}} \zeta\left(E_{e}\right)_{\mathrm{NLO}} \text {, }
$$

$T\left(E_{e}\right)_{\mathrm{N}^{2} \mathrm{LO}}=\frac{1}{1+3 g_{A}^{2}} \frac{E_{0}^{2}}{2 m_{N}^{2}}\left\{\left(\left(-g_{A}^{2}-4(\kappa+1) g_{A}-(\kappa+1)^{2}\right)+\left(g_{A}^{2}+\kappa+1\right) \frac{m_{e}^{2}}{E_{0}^{2}}\right)\right.$

$$
\begin{aligned}
& \left.+\left(5 g_{A}^{2}+(3 \kappa+11) g_{A}+3(\kappa+1)^{2}\right) \frac{E_{e}}{E_{0}}+\left(-22 g_{A}^{2}-26 g_{A}-2(\kappa+1)(\kappa+2)\right) \frac{E_{e}^{2}}{E_{0}^{2}}\right\}+B_{0} \zeta\left(E_{e}\right)_{\mathrm{N}^{2} \mathrm{LO}} \\
& -T\left(E_{e}\right)_{\mathrm{NLO}} \zeta\left(E_{e}\right)_{\mathrm{NLO}}+\frac{8 g_{A}}{1+3 g_{A}^{2}}\left(\frac{E_{0}^{2}}{M_{V}^{2}}+\left(1+2 g_{A}\right) \frac{E_{0}^{2}}{M_{A}^{2}}\right) \frac{E_{e}}{E_{0}}\left(1-\frac{E_{e}}{E_{0}}\right),
\end{aligned}
$$

$U\left(E_{e}\right)_{\mathrm{N}^{2} \mathrm{LO}}=\frac{1}{1+3 g_{A}^{2}} \frac{E_{0}^{2}}{2 m_{N}^{2}} \frac{m_{e}}{E_{0}}\left\{\left((2 \kappa+1) g_{A}+\kappa(\kappa+1)\right)-\kappa\left(g_{A}+\kappa+1\right) \frac{E_{e}}{E_{0}}\right\}$. 
The terms, proportional to $E_{0}^{2} / M_{V}^{2}$ and $E_{0}^{2} / M_{A}^{2}$, are induced by the vector and axial-vector form factors of the neutron beta decay [83] (see also [24]). The slope parameters $M_{V}$ and $M_{A}$ are related to the charge radius of the proton $r_{p}=$ $0.841 \mathrm{fm} \mathrm{[33]} \mathrm{and} \mathrm{the} \mathrm{axial} \mathrm{radius} r_{A}=0.635 \mathrm{fm}$ of the nucleon [84] (see also [85]), respectively. This gives $M_{V}=$ $\sqrt{12} / r_{p}=813 \mathrm{MeV}$ and $M_{A}=\sqrt{12} / r_{A}=1077 \mathrm{MeV}$. The analytical expressions for the corrections $\zeta\left(E_{e}\right)_{\mathrm{NLO}}$, $X\left(E_{e}\right)_{\mathrm{NLO}}$, and $\bar{X}\left(E_{e}\right)_{\mathrm{NLO}}$ for $X=a, A, \ldots, K_{e}, S, T, U$ are given in Eqs. (B2) and (B3), respectively. We define corrections $O\left(E_{e}^{2} / m_{N}^{2}\right)$, caused by weak magnetism and proton recoil, at the level of $10^{-5}$ with the theoretical accuracy of about $10^{-6}$ [46]. The numerical analysis of relative contributions has been carried out for the axial coupling constant $g_{A}=1.2764$ [5], the value of which agrees well with the recommended value of the axial coupling constant obtained by means of the global analysis of the experimental data on the axial coupling constant by Czarnecki et al. [6].

\section{APPENDIX C: WILKINSON'S CORRECTIONS OF ORDER $10^{-5}$ TO THE NEUTRON BETA DECAY}

According to Wilkinson [15], the corrections, additional to those calculated in Appendixes A and B, should be caused by (i) the proton recoil in the electron-proton finalstate Coulomb interaction, (ii) the finite proton radius, (iii) the proton-lepton convolution, and (iv) the higherorder outer radiative corrections. These corrections to the neutron lifetime and the correlation coefficients
$a\left(E_{e}\right), A\left(E_{e}\right), \ldots, K_{e}\left(E_{e}\right)$ have been calculated in $[11,12,14]$. The contributions of the proton recoil, caused by the phase-volume of the neutron beta decay proportional to $1 / m_{N}$ and $1 / m_{N}^{2}$, which are defined in [15] (see also [86]) by the function $S\left(E_{e}, E_{0}, m_{N}\right)$, we have taken into account for the calculation of the NLO and $\mathrm{N}^{2} \mathrm{LO}$ corrections in the large nucleon mass $m_{N}$ expansion induced by weak magnetism and proton recoil (see Appendix B).

\section{Wilkinson's corrections, induced by proton recoil in the Coulomb electron-proton final-state interaction}

For the calculation of the contribution of the proton recoil in the Fermi function we replace the electron velocity $\vec{\beta}$ by a velocity of a relative motion of the electron-proton pair [11]. To NLO in the large nucleon mass $m_{N}$ expansion a velocity of a relative motion of the electron-proton pair is defined by

$$
\vec{\beta} \rightarrow \vec{v}_{\text {rel }}=\vec{\beta}-\frac{\vec{k}_{p}}{m_{N}}
$$

To LO in the large nucleon mass $m_{N}$ expansion the second term in RHS of Eq. (C1) vanishes, and a velocity of a relative motion of the electron-proton pair reduces to an electron velocity. As has been shown in [11] the Fermi function $F\left(E_{e}, Z=1\right)$ with a replacement $\beta \rightarrow v_{\text {rel }}$, caused by a relative motion of the electron-proton pair in the final state of the neutron beta decay, undergoes the following change (see Appendix H of Ref. [11]):

$$
F\left(E_{e}, Z=1\right) \stackrel{\vec{\beta} \rightarrow \vec{r}_{\text {rel }}}{\longrightarrow} F\left(E_{e}, Z=1\right)\left(1-\frac{\pi \alpha}{\beta} \frac{E_{e}}{m_{N}}-\frac{\pi \alpha}{\beta^{3}} \frac{E_{0}-E_{e}}{m_{N}} \frac{\vec{k}_{e} \cdot \vec{k}_{\nu}}{E_{e} E_{\bar{\nu}}}\right)
$$

where we have taken into account the NLO terms in the large nucleon mass $m_{N}$ expansion. The corrections, caused by the proton recoil in the electron-proton final-state interactions to the neutron lifetime $\zeta\left(E_{e}\right)_{\mathrm{WF}}$ and the correlation coefficients $X\left(E_{e}\right)_{\mathrm{WF}}$ for $X=a, A, B, \ldots, T$ and $U$ are equal to

$$
\begin{aligned}
\zeta\left(E_{e}\right)_{\mathrm{WF}} & =-\frac{\pi \alpha}{\beta} \frac{E_{e}}{m_{N}}-\frac{1}{3} a_{0} \frac{\pi \alpha}{\beta} \frac{E_{0}-E_{e}}{m_{N}}=-3.16 \times 10^{-5} \frac{E_{e}}{\beta E_{0}}+1.12 \times 10^{-6} \frac{E_{0}-E_{e}}{\beta E_{0}}, \\
a\left(E_{e}\right)_{\mathrm{WF}} & =\frac{1}{3} a_{0}^{2} \frac{\pi \alpha}{\beta} \frac{E_{0}-E_{e}}{m_{N}}-\frac{\pi \alpha}{\beta^{3}} \frac{E_{0}-E_{e}}{m_{N}}=1.20 \times 10^{-7} \frac{E_{0}-E_{e}}{\beta E_{0}}-3.16 \times 10^{-5} \frac{E_{0}-E_{e}}{\beta^{3} E_{0}}, \\
A\left(E_{e}\right)_{\mathrm{WF}} & =\frac{1}{3} a_{0} A_{0} \frac{\pi \alpha}{\beta} \frac{E_{0}-E_{e}}{m_{N}}=1.35 \times 10^{-7} \frac{E_{0}-E_{e}}{\beta E_{0}}, \\
B\left(E_{e}\right)_{\mathrm{WF}} & =\frac{1}{3} a_{0} B_{0} \frac{\pi \alpha}{\beta} \frac{E_{0}-E_{e}}{m_{N}}=-1.11 \times 10^{-6} \frac{E_{0}-E_{e}}{\beta E_{0}} \\
K_{n}\left(E_{e}\right)_{\mathrm{WF}} & =-A_{0} \frac{\pi \alpha}{\beta^{3}} \frac{E_{0}-E_{e}}{m_{N}}=3.78 \times 10^{-6} \frac{E_{0}-E_{e}}{\beta^{3} E_{0}}, \\
Q_{n}\left(E_{e}\right)_{\mathrm{WF}} & =-B_{0} \frac{\pi \alpha}{\beta^{3}} \frac{E_{0}-E_{e}}{m_{N}}=-3.12 \times 10^{-5} \frac{E_{0}-E_{e}}{\beta^{3} E_{0}},
\end{aligned}
$$




$$
\begin{aligned}
G\left(E_{e}\right)_{\mathrm{WF}} & =-\frac{1}{3} a_{0}\left(1-\beta^{2}\right) \frac{\pi \alpha}{\beta^{3}} \frac{E_{0}-E_{e}}{m_{N}}=1.12 \times 10^{-6}\left(1-\beta^{2}\right) \frac{E_{0}-E_{e}}{\beta^{3} E_{0}}, \\
H\left(E_{e}\right)_{\mathrm{WF}} & =-\frac{1}{3} a_{0}^{2} \sqrt{1-\beta^{2}} \frac{\pi \alpha}{\beta} \frac{E_{0}-E_{e}}{m_{N}}=-1.20 \times 10^{-7} \sqrt{1-\beta^{2}} \frac{E_{0}-E_{e}}{\beta E_{0}}, \\
N\left(E_{e}\right)_{\mathrm{WF}} & =-\frac{1}{3} a_{0} A_{0} \sqrt{1-\beta^{2}} \frac{\pi \alpha}{\beta} \frac{E_{0}-E_{e}}{m_{N}}=1.35 \times 10^{-7} \sqrt{1-\beta^{2}} \frac{E_{0}-E_{e}}{\beta E_{0}}, \\
Q_{e}\left(E_{e}\right)_{\mathrm{WF}} & =-\frac{1}{3} a_{0} A_{0} \frac{\pi \alpha}{\beta} \frac{E_{0}-E_{e}}{m_{N}}+\frac{1}{3} B_{0}\left(1+\sqrt{1-\beta^{2}}\right) \frac{\pi \alpha}{\beta^{3}} \frac{E_{0}-E_{e}}{m_{N}} \\
& =-1.35 \times 10^{-7} \frac{E_{0}-E_{e}}{\beta E_{0}}+1.04 \times 10^{-4}\left(1+\sqrt{1-\beta^{2}}\right) \frac{E_{0}-E_{e}}{\beta^{3} E_{0}} \\
K_{e}\left(E_{e}\right)_{\mathrm{WF}} & =-\frac{1}{3} a_{0}^{2} \frac{\pi \alpha}{\beta} \frac{E_{0}-E_{e}}{m_{N}}\left(1+\sqrt{1-\beta^{2}}\right) \frac{\pi \alpha}{\beta^{3}} \frac{E_{0}-E_{e}}{m_{N}} \\
& =-1.20 \times 10^{-7} \frac{E_{0}-E_{e}}{\beta E_{0}}+3.16 \times 10^{-5}\left(1+\sqrt{1-\beta^{2}}\right) \frac{E_{0}-E_{e}}{\beta^{3} E_{0}}, \\
S\left(E_{e}\right)_{\mathrm{WF}} & =\sqrt{1-\beta^{2} A_{0}} \frac{\pi \alpha}{\beta^{3}} \frac{E_{0}-E_{e}}{m_{N}}=3.78 \times 10^{-6} \sqrt{1-\beta^{2}} \frac{E_{0}-E_{e}}{\beta^{3} E_{0}}, \\
T\left(E_{e}\right)_{\mathrm{WF}} & =-\frac{1}{3} a_{0} B_{0} \frac{\pi \alpha}{\beta} \frac{E_{0}-E_{e}}{m_{N}}=1.11 \times 10^{-6} \frac{E_{0}-E_{e}}{\beta E_{0}}, \\
U\left(E_{e}\right)_{\mathrm{WF}} & =0 .
\end{aligned}
$$

In comparison with the result, obtained in [12], an additional term in $Q_{e}\left(E_{e}\right)_{\mathrm{WF}}$ appears because of the contribution off the correlation coefficient $T\left(E_{e}\right)$. The contributions of Wilkinson's corrections, caused by proton recoil in the electron-proton final-state Coulomb interaction, to the electron-energy and angular distribution of the neutron beta decay with correlation structures beyond the standard correlation structures by Jackson et al. [25] and Ebel and Feldman [28] [see Eq. (1)] are given in Appendix F. We would like to emphasize that Wilkinson's corrections, induced by proton recoil in the final-state Coulomb electron-proton interaction are well-defined in the experimental electron-energy region $0.811 \mathrm{MeV} \leq E_{e} \leq 1.211 \mathrm{MeV}$ [5].
2. Wilkinson's corrections, induced by (i) the finite proton-radius $r_{p}$, (ii) the lepton-nucleon convolution, and (iii) the higher-order outer radiative corrections

The corrections under consideration, caused by (i) the finite proton-radius $r_{p}$, (ii) the lepton-nucleon convolution, and (iii) the higher-order outer radiative corrections, are defined by the functions $L\left(E_{e}, Z=1\right), C\left(E_{e}, Z=1\right)$, and $J(Z=1)$, respectively [15] (see also [12]). According to Wilkinson [15], the contribution of $J(Z=1)$ is equal to $J(Z=1)=1+3.92 \times 10^{-4}$ (see also [12]). The corrections $L\left(E_{e}, Z=1\right)$ and $C\left(E_{e}, Z=1\right)$, adapted for the neutron beta decay, are determined by [12]

$$
\begin{aligned}
L\left(E_{e}, Z=1\right)=1 & +\frac{13}{60} \alpha^{2}-\alpha r_{p} E_{e}\left(1-\frac{1}{2} \frac{m_{e}^{2}}{E_{e}^{2}}\right)=1+1.15 \times 10^{-5}-4.02 \times 10^{-5} \frac{E_{e}}{E_{0}}, \\
C\left(E_{e}, Z=1\right)=1+ & {\left[\left(-\frac{9}{20} \alpha^{2}+\frac{1}{5} m_{e}^{2} r_{p}^{2}-\frac{1}{5} E_{0}^{2} r_{p}^{2}\right)+\left(-\frac{1}{5} \alpha r_{p} E_{0}-\frac{2}{15} E_{0}^{2} r_{p}^{2}\right) \frac{1-g_{A}^{2}}{1+3 g_{A}^{2}}\right] } \\
& +\left[\left(-\frac{3}{5} \alpha r_{p} E_{0}+\frac{2}{5} E_{0}^{2} r_{p}^{2}\right)+\left(\frac{1}{5} \alpha r_{p} E_{0}-\frac{2}{15} E_{0}^{2} r_{p}^{2}\right) \frac{1-g_{A}^{2}}{1+3 g_{A}^{2}}\right] \frac{E_{e}}{E_{0}}+\frac{2}{15} m_{e} E_{0} r_{p}^{2} \frac{1-g_{A}^{2}}{1+3 g_{A}^{2}} \frac{m_{e}}{E_{e}} \\
& +\frac{2}{5}\left(-1+\frac{1}{3} \frac{1-g_{A}^{2}}{1+3 g_{A}^{2}}\right) E_{0}^{2} r_{p}^{2} \frac{E_{e}^{2}}{E_{0}^{2}}=1-2.78 \times 10^{-5}-1.24 \times 10^{-5} \frac{E_{e}}{E_{0}}-1.26 \times 10^{-5} \frac{E_{e}^{2}}{E_{0}^{2}} .
\end{aligned}
$$

For the calculation of the numerical values of the constant terms and coefficients in front of the powers of $E_{e} / E_{0}$ we use $r_{p}=0.841 \mathrm{fm}$ [33]. Following Wilkinson [15] we define Wilkinson's correction, caused by (i) the finite proton-radius $r_{p}$, (ii) the lepton-nucleon convolution, and (iii) the higher-order outer radiative corrections, as follows:

$$
L\left(E_{e}, Z=1\right) C\left(E_{e}, Z=1\right) J(Z=1)=1+\delta L\left(E_{e}, Z=1\right)+\delta C\left(E_{e}, Z=1\right)+\delta J(Z=1)=1+\zeta\left(E_{e}\right)_{\mathrm{WR}}
$$


where $\zeta\left(E_{e}\right)_{\mathrm{WR}}$ is equal to

$$
\zeta\left(E_{e}\right)_{\mathrm{WR}}=3.76 \times 10^{-4}-5.26 \times 10^{-5} \frac{E_{e}}{E_{0}}-1.26 \times 10^{-5} \frac{E_{e}^{2}}{E_{0}^{2}}
$$

The contributions of Wilkinson's corrections, caused by (i) the finite proton-radius $r_{p}$, (ii) the lepton-nucleon convolution, and (iii) the higher-order outer radiative corrections, to the correlation coefficients are equal to

$$
\begin{aligned}
& a\left(E_{e}\right)_{\mathrm{WR}}=-a_{0} \zeta\left(E_{e}\right)_{\mathrm{WR}}=-a_{0}\left(3.76 \times 10^{-4}-5.26 \times 10^{-5} \frac{E_{e}}{E_{0}}-1.26 \times 10^{-5} \frac{E_{e}^{2}}{E_{0}^{2}}\right), \\
& A\left(E_{e}\right)_{\mathrm{WR}}=-A_{0} \zeta\left(E_{e}\right)_{\mathrm{WR}}=-A_{0}\left(3.76 \times 10^{-4}-5.26 \times 10^{-5} \frac{E_{e}}{E_{0}}-1.26 \times 10^{-5} \frac{E_{e}^{2}}{E_{0}^{2}}\right), \\
& B\left(E_{e}\right)_{\mathrm{WR}}=-B_{0} \zeta\left(E_{e}\right)_{\mathrm{WR}}=-B_{0}\left(3.76 \times 10^{-4}-5.26 \times 10^{-5} \frac{E_{e}}{E_{0}}-1.26 \times 10^{-5} \frac{E_{e}^{2}}{E_{0}^{2}}\right), \\
& K_{n}\left(E_{e}\right)_{\mathrm{WR}}=Q_{n}\left(E_{e}\right)_{\mathrm{WR}}=0, \\
& G\left(E_{e}\right)_{\mathrm{WR}}=\zeta\left(E_{e}\right)_{\mathrm{WR}}=3.76 \times 10^{-4}-5.26 \times 10^{-5} \frac{E_{e}}{E_{0}}-1.26 \times 10^{-5} \frac{E_{e}^{2}}{E_{0}^{2}}, \\
& H\left(E_{e}\right)_{\mathrm{WR}}=\frac{m_{e}}{E_{e}} a_{0} \zeta\left(E_{e}\right)_{\mathrm{WR}}=\frac{m_{e}}{E_{0}} a_{0}\left(3.76 \times 10^{-4} \frac{E_{0}}{E_{e}}-5.26 \times 10^{-5}-1.26 \times 10^{-5} \frac{E_{e}}{E_{0}}\right), \\
& N\left(E_{e}\right)_{\mathrm{WR}}=\frac{m_{e}}{E_{e}} A_{0} \zeta\left(E_{e}\right)_{\mathrm{WR}}=\frac{m_{e}}{E_{0}} A_{0}\left(3.76 \times 10^{-4} \frac{E_{0}}{E_{e}}-5.26 \times 10^{-5}-1.26 \times 10^{-5} \frac{E_{e}}{E_{0}}\right), \\
& Q_{e}\left(E_{e}\right)_{\mathrm{WR}}=A_{0} \zeta\left(E_{e}\right)_{\mathrm{WR}}=A_{0}\left(3.76 \times 10^{-4}-5.26 \times 10^{-5} \frac{E_{e}}{E_{0}}-1.26 \times 10^{-5} \frac{E_{e}^{2}}{E_{0}^{2}}\right), \\
& K_{e}\left(E_{e}\right)_{\mathrm{WR}}=a_{0} \zeta\left(E_{e}\right)_{\mathrm{WR}}=a_{0}\left(3.76 \times 10^{-4}-5.26 \times 10^{-5} \frac{E_{e}}{E_{0}}-1.26 \times 10^{-5} \frac{E_{e}^{2}}{E_{0}^{2}}\right), \\
& S\left(E_{e}\right)_{\mathrm{WR}}=U\left(E_{e}\right)_{\mathrm{WR}}=0, \\
& T\left(E_{e}\right)_{\mathrm{WR}}=B_{0} \zeta\left(E_{e}\right)_{\mathrm{WR}}=B_{0}\left(3.76 \times 10^{-4}-5.26 \times 10^{-5} \frac{E_{e}}{E_{0}}-1.26 \times 10^{-5} \frac{E_{e}^{2}}{E_{0}^{2}}\right) .
\end{aligned}
$$

Now we may obtain the total contributions of Wilkinson's corrections to the correlation function $\zeta\left(E_{e}\right)$ and the correlation coefficients. We would like to emphasize that the correction $\zeta\left(E_{e}\right)_{\mathrm{WR}}$ does not depend practically on the value of the axial coupling constant $g_{A}$.

\section{Wilkinson's corrections to the neutron beta decay}

Summing the contributions in Eqs. (C3), (C6), and (C7) we define total Wilkinson's corrections to the neutron lifetime and the correlation coefficients of the neutron beta decay

$$
\begin{aligned}
\zeta\left(E_{e}\right)_{\mathrm{WC}} & =3.76 \times 10^{-4}-5.26 \times 10^{-5} \frac{E_{e}}{E_{0}}-1.26 \times 10^{-5} \frac{E_{e}^{2}}{E_{0}^{2}}-\frac{\pi \alpha}{\beta} \frac{E_{e}}{m_{N}}, \\
a\left(E_{e}\right)_{\mathrm{WC}} & =-3.76 \times 10^{-4} a_{0}-\frac{\pi \alpha}{\beta^{3}} \frac{E_{0}-E_{e}}{m_{N}}, \\
A\left(E_{e}\right)_{\mathrm{WC}} & =-3.76 \times 10^{-4} A_{0}, \\
B\left(E_{e}\right)_{\mathrm{WC}} & =-B_{0}\left(3.76 \times 10^{-4}-5.26 \times 10^{-5} \frac{E_{e}}{E_{0}}-1.26 \times 10^{-5} \frac{E_{e}^{2}}{E_{0}^{2}}\right), \\
K_{n}\left(E_{e}\right)_{\mathrm{WC}} & =0
\end{aligned}
$$




$$
\begin{aligned}
Q_{n}\left(E_{e}\right)_{\mathrm{WC}} & =-B_{0} \frac{\pi \alpha}{\beta^{3}} \frac{E_{0}-E_{e}}{\beta^{3} E_{0}}, \\
G\left(E_{e}\right)_{\mathrm{WC}} & =3.76 \times 10^{-4}-5.26 \times 10^{-5} \frac{E_{e}}{E_{0}}-1.26 \times 10^{-5} \frac{E_{e}^{2}}{E_{0}^{2}}, \\
H\left(E_{e}\right)_{\mathrm{WC}} & =N\left(E_{e}\right)_{\mathrm{WC}}=0, \\
Q_{e}\left(E_{e}\right)_{\mathrm{WC}} & =3.76 \times 10^{-4} A_{0}+\frac{1}{3} B_{0}\left(1+\sqrt{1-\beta^{2}}\right) \frac{\pi \alpha}{\beta^{3}} \frac{E_{0}-E_{e}}{m_{N}}, \\
K_{e}\left(E_{e}\right)_{\mathrm{WC}} & =3.76 \times 10^{-4} a_{0}+\left(1+\sqrt{1-\beta^{2}}\right) \frac{\pi \alpha}{\beta^{3}} \frac{E_{0}-E_{e}}{m_{N}}, \\
S\left(E_{e}\right)_{\mathrm{WC}} & =U\left(E_{e}\right)_{\mathrm{W}}=0, \\
T\left(E_{e}\right)_{\mathrm{WC}} & =B_{0}\left(3.76 \times 10^{-4}-5.26 \times 10^{-5} \frac{E_{e}}{E_{0}}-1.26 \times 10^{-5} \frac{E_{e}^{2}}{E_{0}^{2}}\right) .
\end{aligned}
$$

Wilkinson's corrections in Eq. (C8) are calculated at the account for the contributions, which are not smaller than a few parts of $10^{-5}$ with a theoretical accuracy of about a few parts of $10^{-6}$ in the experimental electron-energy region $0.811 \mathrm{MeV} \leq$ $E_{e} \leq 1.211 \mathrm{MeV}[5]$.

\section{APPENDIX D: RADIATIVE CORRECTIONS OF ORDER $O\left(\alpha E_{e} / m_{N}\right)$, INDUCED BY SIRLIN'S OUTER RADIATIVE CORRECTIONS OF ORDER $O(\alpha / \pi)$ AND THE PHASE-VOLUME OF THE NEUTRON BETA DECAY}

In this Appendix we analyze the contributions of the radiative corrections $O\left(\alpha E_{e} / m_{N}\right)$, induced by Sirlin's outer radiative corrections of order $O(\alpha / \pi)$ and the phase-volume of the neutron beta decay taken to NLO in the large nucleon mass $m_{N}$ expansion. For this aim we rewrite Eq. (A13) keeping the contributions of the $O(\alpha / \pi)$ corrections only. We get

$$
\begin{aligned}
\sum_{\text {pol }} \frac{\left|M\left(n \rightarrow p e^{-} \bar{\nu}_{e}\right)\right|^{2}}{\left(1+3 g_{A}^{2}\right)\left|G_{V}\right|^{2} 64 m_{n}^{2} E_{e} E_{\bar{\nu}}}= & {\left[\left(1+\frac{\alpha}{\pi} f_{\beta}\left(E_{e}, \mu\right)\right)+B_{0}\left(1+\frac{\alpha}{\pi} f_{\beta}\left(E_{e}, \mu\right)\right) \frac{\vec{\xi}_{n} \cdot \vec{k}_{\bar{\nu}}}{E_{\bar{\nu}}}\right]\left(1-\frac{m_{e}}{E_{e}} \zeta_{e}^{0}\right) } \\
& +\left[a_{0}\left(1+\frac{\alpha}{\pi} f_{\beta}\left(E_{e}, \mu\right)\right) \frac{\vec{k}_{\bar{\nu}}}{E_{\bar{\nu}}}+A_{0}\left(1+\frac{\alpha}{\pi} f_{\beta}\left(E_{e}, \mu\right)\right) \vec{\xi}_{n}\right] \cdot\left(\frac{\vec{k}_{e}}{E_{e}}-\frac{m_{e}}{E_{e}} \vec{\zeta}_{e}\right) \\
& +\left[-\frac{\alpha}{\pi} \frac{\sqrt{1-\beta^{2}}}{2 \beta} \ell n\left(\frac{1+\beta}{1-\beta}\right)-B_{0} \frac{\alpha}{\pi} \frac{\sqrt{1-\beta^{2}}}{2 \beta} \ell n\left(\frac{1+\beta}{1-\beta}\right) \frac{\vec{\xi}_{n} \cdot \vec{k}_{\bar{\nu}}}{E_{\bar{\nu}}}\right] \frac{m_{e}}{E_{e}} \\
& +\left[A_{0} \frac{\alpha}{\pi} \frac{\sqrt{1-\beta^{2}}}{2 \beta} \ell n\left(\frac{1+\beta}{1-\beta}\right) \vec{\xi}_{n}+a_{0} \frac{\alpha \sqrt{1-\beta^{2}}}{\pi} \ell n\left(\frac{1+\beta}{1-\beta}\right) \frac{\vec{k}_{\bar{\nu}}}{E_{\bar{\nu}}}\right] \cdot\left(\vec{\zeta}_{e}-\frac{\vec{k}_{e}}{E_{e}} \zeta_{e}^{0}\right) \\
& +\cdots,
\end{aligned}
$$

where the ellipsis denotes the contributions, which are not important for the aim of this Appendix. In terms of irreducible correlation structures and using Eq. (A7) we transcribe the RHS of Eq. (D1) into the form

$$
\begin{aligned}
\sum_{\text {pol }} & \frac{\left|M\left(n \rightarrow p e^{-} \bar{\nu}_{e}\right)\right|^{2}}{\left(1+3 g_{A}^{2}\right)\left|G_{V}\right|^{2} 64 m_{n}^{2} E_{e} E_{\bar{\nu}}} \\
= & {\left[1+\frac{\alpha}{\pi}\left(\bar{g}_{n}\left(E_{e}\right)-g_{\beta \gamma}^{(1)}\left(E_{e}, \mu\right)\right)\right]+a_{0}\left[1+\frac{\alpha}{\pi}\left(\bar{g}_{n}\left(E_{e}\right)+\frac{\sqrt{1-\beta^{2}}}{2 \beta} \ell\left(\frac{1+\beta}{1-\beta}\right)-g_{\beta \gamma}^{(1)}\left(E_{e}, \mu\right)\right)\right] \frac{\vec{k}_{e} \cdot \vec{k}_{\bar{\nu}}}{E_{e} E_{\bar{\nu}}} } \\
& +A_{0}\left[1+\frac{\alpha}{\pi}\left(\bar{g}_{n}\left(E_{e}\right)+\frac{1-\beta^{2}}{2 \beta} \ell n\left(\frac{1+\beta}{1-\beta}\right)-g_{\beta \gamma}^{(1)}\left(E_{e}, \mu\right)\right)\right] \frac{\vec{\xi}_{n} \cdot \vec{k}_{e}}{E_{e}}+B_{0}\left[1+\frac{\alpha}{\pi}\left(\bar{g}_{n}\left(E_{e}\right)-g_{\beta \gamma}^{(1)}\left(E_{e}, \mu\right)\right)\right] \frac{\vec{\xi}_{n} \cdot \vec{k}_{\bar{\nu}}}{E_{\bar{\nu}}}
\end{aligned}
$$




$$
\begin{aligned}
& -\left[1+\frac{\alpha}{\pi}\left(\bar{g}_{n}\left(E_{e}\right)+\frac{1-\beta^{2}}{2 \beta} \ell n\left(\frac{1+\beta}{1-\beta}\right)-g_{\beta \gamma}^{(1)}\left(E_{e}, \mu\right)\right)\right] \frac{\vec{\xi}_{e} \cdot \vec{k}_{e}}{E_{e}} \\
& -\frac{m_{e}}{E_{e}} a_{0}\left[1+\frac{\alpha}{\pi}\left(\bar{g}_{n}\left(E_{e}\right)-\frac{\beta}{2} \ell n\left(\frac{1+\beta}{1-\beta}\right)-g_{\beta \gamma}^{(1)}\left(E_{e}, \mu\right)\right)\right] \frac{\vec{\xi}_{e} \cdot \vec{k}_{\bar{\nu}}}{E_{\bar{\nu}}} \\
& -\frac{m_{e}}{E_{e}} A_{0}\left[1+\frac{\alpha}{\pi}\left(\bar{g}_{n}\left(E_{e}\right)-\frac{\beta}{2} \ell n\left(\frac{1+\beta}{1-\beta}\right)-g_{\beta \gamma}^{(1)}\left(E_{e}, \mu\right)\right)\right] \vec{\xi}_{n} \cdot \vec{\xi}_{e} \\
& -A_{0}\left[1+\frac{\alpha}{\pi}\left(\bar{g}_{n}\left(E_{e}\right)+\left(1+\sqrt{1-\beta^{2}}\right) \frac{\sqrt{1-\beta^{2}}}{2 \beta} \ell n\left(\frac{1+\beta}{1-\beta}\right)-g_{\beta \gamma}^{(1)}\left(E_{e}, \mu\right)\right)\right] \frac{\left(\vec{\xi}_{n} \cdot \vec{k}_{e}\right)\left(\vec{k}_{e} \cdot \vec{\xi}_{e}\right)}{\left(E_{e}+m_{e}\right) E_{e}} \\
& -a_{0}\left[1+\frac{\alpha}{\pi}\left(\bar{g}_{n}\left(E_{e}\right)+\left(1+\sqrt{1-\beta^{2}}\right) \frac{\sqrt{1-\beta^{2}}}{2 \beta} \ell n\left(\frac{1+\beta}{1-\beta}\right)-g_{\beta \gamma}^{(1)}\left(E_{e}, \mu\right)\right)\right] \frac{\left(\vec{\xi}_{e} \cdot \vec{k}_{e}\right)\left(\vec{k}_{e} \cdot \vec{k}_{\bar{\nu}}\right)}{\left(E_{e}+m_{e}\right) E_{e} E_{\bar{\nu}}} \\
& -B_{0}\left(1+\frac{\alpha}{\pi}\left(\bar{g}_{n}\left(E_{e}\right)+\frac{1-\beta^{2}}{2 \beta} \ell n\left(\frac{1+\beta}{1-\beta}\right)-g_{\beta \gamma}^{(1)}\left(E_{e}, \mu\right)\right)\right) \frac{\left(\vec{\xi}_{n} \cdot \vec{k}_{\bar{\nu}}\right)\left(\vec{\xi}_{e} \cdot \vec{k}_{e}\right)}{E_{e} E_{\bar{\nu}}} .
\end{aligned}
$$

Taking into account the contribution of the phase-volume of the neutron beta decay Eq. (A2) we obtain

$$
\begin{aligned}
\Phi_{n}\left(\vec{k}_{e}, \vec{k}_{\bar{\nu}_{e}}\right) \sum_{\mathrm{pol}} \frac{\left|M\left(n \rightarrow p e^{-} \bar{\nu}_{e}\right)\right|^{2}}{\left(1+3 g_{A}^{2}\right)\left|G_{V}\right|^{2} 64 m_{n}^{2} E_{e} E_{\bar{\nu}}} & {\left[1+3 \frac{E_{e}}{m_{N}}\left(1-\frac{\vec{k}_{e} \cdot \vec{k}_{\bar{\nu}}}{E_{e} E_{\bar{\nu}}}\right)\right]\left\{\left[1+\frac{\alpha}{\pi}\left(\bar{g}_{n}\left(E_{e}\right)-g_{\beta \gamma}^{(1)}\left(E_{e}, \mu\right)\right)\right]\right.} \\
& +a_{0}\left[1+\frac{\alpha}{\pi}\left(\bar{g}_{n}\left(E_{e}\right)+\frac{\sqrt{1-\beta^{2}}}{2 \beta} \ell n\left(\frac{1+\beta}{1-\beta}\right)-g_{\beta \gamma}^{(1)}\left(E_{e}, \mu\right)\right)\right] \frac{\vec{k}_{e} \cdot \vec{k}_{\bar{\nu}}}{E_{e} E_{\bar{\nu}}} \\
& +A_{0}\left[1+\frac{\alpha}{\pi}\left(\bar{g}_{n}\left(E_{e}\right)+\frac{1-\beta^{2}}{2 \beta} \ell n\left(\frac{1+\beta}{1-\beta}\right)-g_{\beta \gamma}^{(1)}\left(E_{e}, \mu\right)\right)\right] \frac{\vec{\xi}_{n} \cdot \vec{k}_{e}}{E_{e}} \\
& +B_{0}\left[1+\frac{\alpha}{\pi}\left(\bar{g}_{n}\left(E_{e}\right)-g_{\beta \gamma}^{(1)}\left(E_{e}, \mu\right)\right)\right] \frac{\vec{\xi}_{n} \cdot \vec{k}_{\bar{\nu}}}{E_{\bar{\nu}}} \\
& -\left[1+\frac{\alpha}{\pi}\left(\bar{g}_{n}\left(E_{e}\right)+\frac{1-\beta^{2}}{2 \beta} \ell n\left(\frac{1+\beta}{1-\beta}\right)-g_{\beta \gamma}^{(1)}\left(E_{e}, \mu\right)\right)\right] \frac{\vec{\xi}_{e} \cdot \vec{k}_{e}}{E_{e}} \\
& -\frac{m_{e}}{E_{e}} a_{0}\left[1+\frac{\alpha}{\pi}\left(\bar{g}_{n}\left(E_{e}\right)-\frac{\beta}{2} \ell n\left(\frac{1+\beta}{1-\beta}\right)-g_{\beta \gamma}^{(1)}\left(E_{e}, \mu\right)\right)\right] \frac{\vec{\xi}_{e} \cdot \vec{k}_{\bar{\nu}}}{E_{\bar{\nu}}} \\
& -\frac{m_{e}}{E_{e}} A_{0}\left[1+\frac{\alpha}{\pi}\left(\bar{g}_{n}\left(E_{e}\right)-\frac{\beta}{2} \ell n\left(\frac{1+\beta}{1-\beta}\right)-g_{\beta \gamma}^{(1)}\left(E_{e}, \mu\right)\right)\right] \vec{\xi}_{n} \cdot \vec{\xi}_{e} \\
& -A_{0}\left[1+\frac{\alpha}{\pi}\left(\bar{g}_{n}\left(E_{e}\right)+\left(1+\sqrt{1-\beta^{2}}\right) \frac{\sqrt{1-\beta^{2}}}{2 \beta} \ell n\left(\frac{1+\beta}{1-\beta}\right)-g_{\beta \gamma}^{(1)}\left(E_{e}, \mu\right)\right)\right] \\
& \times \frac{\left(\vec{\xi}_{n} \cdot \vec{k}_{e}\right)\left(\vec{k}_{e} \cdot \vec{\xi}_{e}\right)}{\left(E_{e}+m_{e}\right) E_{e}}-a_{0}\left[1+\frac{\alpha}{\pi}\left(\bar{g}_{n}\left(E_{e}\right)+\left(1+\sqrt{1-\beta^{2}}\right) \frac{\sqrt{1-\beta^{2}}}{2 \beta} \ell n\left(\frac{1+\beta}{1-\beta}\right)\right.\right. \\
& \left.\left.-g_{\beta \gamma}^{(1)}\left(E_{e}, \mu\right)\right)\right] \frac{\left(\vec{\xi}_{e} \cdot \vec{k}_{e}\right)\left(\vec{k}_{e} \cdot \vec{k}_{\bar{\nu}}\right)}{\left(E_{e}+m_{e}\right) E_{e} E_{\bar{\nu}}} \\
& \left.-B_{0}\left[1+\frac{\alpha}{\pi}\left(\bar{g}_{n}\left(E_{e}\right)+\frac{1-\beta^{2}}{2 \beta} \ell n\left(\frac{1+\beta}{1-\beta}\right)-g_{\beta \gamma}^{(1)}\left(E_{e}, \mu\right)\right)\right] \frac{\left(\vec{\xi}_{n} \cdot \vec{k}_{\bar{\nu}}\right)\left(\vec{\xi}_{e} \cdot \vec{k}_{e}\right)}{E_{e} E_{\bar{\nu}}}\right\} .
\end{aligned}
$$

The electron-energy and angular distribution of the neutron beta decay, taking into account the radiative corrections of order $O(\alpha / \pi)$ and the NLO corrections in the large nucleon mass $m_{N}$ expansion, induced by the phase-volume, is given by 


$$
\begin{aligned}
& \frac{d^{5} \lambda_{\beta}\left(E_{e}, \vec{k}_{e}, \vec{k}_{\bar{\nu}}, \vec{\xi}_{n}, \vec{\xi}_{e}\right)}{d E_{e} d \Omega_{e} d \Omega_{\bar{\nu}}} \\
& =\left(1+3 g_{A}^{2}\right) \frac{\left|G_{V}\right|^{2}}{16 \pi^{5}}\left(E_{0}-E_{e}\right)^{2} \sqrt{E_{e}^{2}-m_{e}^{2}} E_{e} F\left(E_{e}, Z=1\right)\left[1+3 \frac{E_{e}}{m_{N}}\left(1-\frac{\vec{k}_{e} \cdot \vec{k}_{\bar{\nu}}}{E_{e} E_{\bar{\nu}}}\right)\right] \\
& \times\left\{\left[1+\frac{\alpha}{\pi}\left(\bar{g}_{n}\left(E_{e}\right)-g_{\beta \gamma}^{(1)}\left(E_{e}, \mu\right)\right)\right]+a_{0}\left[1+\frac{\alpha}{\pi}\left(\bar{g}_{n}\left(E_{e}\right)+\frac{\sqrt{1-\beta^{2}}}{2 \beta} \ell n\left(\frac{1+\beta}{1-\beta}\right)-g_{\beta \gamma}^{(1)}\left(E_{e}, \mu\right)\right)\right] \frac{\vec{k}_{e} \cdot \vec{k}_{\bar{\nu}}}{E_{e} E_{\bar{\nu}}}\right. \\
& +A_{0}\left[1+\frac{\alpha}{\pi}\left(\bar{g}_{n}\left(E_{e}\right)+\frac{1-\beta^{2}}{2 \beta} \ell n\left(\frac{1+\beta}{1-\beta}\right)-g_{\beta \gamma}^{(1)}\left(E_{e}, \mu\right)\right)\right] \frac{\vec{\xi}_{n} \cdot \vec{k}_{e}}{E_{e}}+B_{0}\left[1+\frac{\alpha}{\pi}\left(\bar{g}_{n}\left(E_{e}\right)-g_{\beta \gamma}^{(1)}\left(E_{e}, \mu\right)\right)\right] \frac{\vec{\xi}_{n} \cdot \vec{k}_{\bar{\nu}}}{E_{\bar{\nu}}} \\
& -\left[1+\frac{\alpha}{\pi}\left(\bar{g}_{n}\left(E_{e}\right)+\frac{1-\beta^{2}}{2 \beta} \ell n\left(\frac{1+\beta}{1-\beta}\right)-g_{\beta \gamma}^{(1)}\left(E_{e}, \mu\right)\right)\right] \frac{\vec{\xi}_{e} \cdot \vec{k}_{e}}{E_{e}}-\frac{m_{e}}{E_{e}} a_{0}\left[1+\frac{\alpha}{\pi}\left(\bar{g}_{n}\left(E_{e}\right)-\frac{\beta}{2} \ell n\left(\frac{1+\beta}{1-\beta}\right)-g_{\beta \gamma}^{(1)}\left(E_{e}, \mu\right)\right)\right] \\
& \times \frac{\vec{\xi}_{e} \cdot \vec{k}_{\bar{\nu}}}{E_{\bar{\nu}}}-\frac{m_{e}}{E_{e}} A_{0}\left[1+\frac{\alpha}{\pi}\left(\bar{g}_{n}\left(E_{e}\right)-\frac{\beta}{2} \ell n\left(\frac{1+\beta}{1-\beta}\right)-g_{\beta \gamma}^{(1)}\left(E_{e}, \mu\right)\right)\right] \vec{\xi}_{n} \cdot \vec{\xi}_{e}-A_{0}\left[1+\frac{\alpha}{\pi}\left(\bar{g}_{n}\left(E_{e}\right)+\left(1+\sqrt{1-\beta^{2}}\right)\right.\right. \\
& \left.\left.\times \frac{\sqrt{1-\beta^{2}}}{2 \beta} \ell n\left(\frac{1+\beta}{1-\beta}\right)-g_{\beta \gamma}^{(1)}\left(E_{e}, \mu\right)\right)\right] \frac{\left(\vec{\xi}_{n} \cdot \vec{k}_{e}\right)\left(\vec{k}_{e} \cdot \vec{\xi}_{e}\right)}{\left(E_{e}+m_{e}\right) E_{e}}-a_{0}\left[1+\frac{\alpha}{\pi}\left(\bar{g}_{n}\left(E_{e}\right)+\left(1+\sqrt{1-\beta^{2}}\right) \frac{\sqrt{1-\beta^{2}}}{2 \beta} \ell n\left(\frac{1+\beta}{1-\beta}\right)\right.\right. \\
& \left.\left.\left.-g_{\beta \gamma}^{(1)}\left(E_{e}, \mu\right)\right)\right] \frac{\left(\vec{\xi}_{e} \cdot \vec{k}_{e}\right)\left(\vec{k}_{e} \cdot \vec{k}_{\bar{\nu}}\right)}{\left(E_{e}+m_{e}\right) E_{e} E_{\bar{\nu}}}-B_{0}\left[1+\frac{\alpha}{\pi}\left(\bar{g}_{n}\left(E_{e}\right)+\frac{1-\beta^{2}}{2 \beta} \ell n\left(\frac{1+\beta}{1-\beta}\right)-g_{\beta \gamma}^{(1)}\left(E_{e}, \mu\right)\right)\right] \frac{\left(\vec{\xi}_{n} \cdot \vec{k}_{\bar{\nu}}\right)\left(\vec{\xi}_{e} \cdot \vec{k}_{e}\right)}{E_{e} E_{\bar{\nu}}}\right\} .
\end{aligned}
$$

In order to remove the dependence of the electron-energy and angular distribution of the neutron beta decay on the infrared cutoff $\mu$ we have to take into account the contribution of the neutron radiative beta decay $n \rightarrow p+e^{-}+\bar{\nu}_{e}+\gamma$, where $\gamma$ is a real photon. It is well-known [57-61] (see also [19,21] and $[11,12,14]$ ) that the contribution of the neutron radiative beta decay is extremely needed for cancellation of the infrared divergences in the radiative corrections of order $O(\alpha / \pi)$, caused by one-virtual photon exchanges.

For the removal of the infrared dependence we use the following electron-photon-energy and angular distribution, calculated in [30] [see Eq. (B-14) in Ref. [30] ]:

$$
\begin{aligned}
& d^{6} \lambda_{\beta \gamma}\left(E_{e}, \omega, \vec{k}_{e}, \vec{k}_{\bar{\nu}}, \vec{\xi}_{n}, \vec{\xi}_{e}\right) \\
& d \omega d E_{e} d \Omega_{e} d \Omega_{\bar{\nu}} \\
& =\left(1+3 g_{A}^{2}\right) \frac{\alpha}{\pi} \frac{\left|G_{V}\right|^{2}}{16 \pi^{5}} \sqrt{E_{e}^{2}-m_{e}^{2}} E_{e} F\left(E_{e}, Z=1\right)\left(E_{0}-E_{e}-\omega\right)^{2} \Phi_{n \gamma}\left(\vec{k}_{e}, \vec{k}_{\bar{\nu}_{e}}, \omega\right) \\
& \times\left\{\frac{1}{\omega}\left[\left(1+\frac{\omega}{E_{e}}+\frac{1}{2} \frac{\omega^{2}}{E_{e}^{2}}\right)\left[\frac{1}{\beta} \ell n\left(\frac{1+\beta}{1-\beta}\right)-2\right]+\frac{\omega^{2}}{E_{e}^{2}}\right]+a_{0} \frac{\vec{k}_{e} \cdot \vec{k}_{\bar{\nu}}}{E_{e} E_{\bar{\nu}}} \frac{1}{\omega}\left(1+\frac{1}{\beta^{2}} \frac{\omega}{E_{e}}+\frac{1}{2 \beta^{2}} \frac{\omega^{2}}{E_{e}^{2}}\right)\left[\frac{1}{\beta} \ell n\left(\frac{1+\beta}{1-\beta}\right)-2\right]\right. \\
& +A_{0} \frac{\vec{\xi}_{n} \cdot \vec{k}_{e}}{E_{e}} \frac{1}{\omega}\left(1+\frac{1}{\beta^{2}} \frac{\omega}{E_{e}}+\frac{1}{2 \beta^{2}} \frac{\omega^{2}}{E_{e}^{2}}\right)\left[\frac{1}{\beta} \ell n\left(\frac{1+\beta}{1-\beta}\right)-2\right]+B_{0} \frac{\vec{\xi}_{n} \cdot \vec{k}_{\bar{\nu}}}{E_{\bar{\nu}}} \frac{1}{\omega}\left[\left(1+\frac{\omega}{E_{e}}+\frac{1}{2} \frac{\omega^{2}}{E_{e}^{2}}\right)\left[\frac{1}{\beta} \ell n\left(\frac{1+\beta}{1-\beta}\right)-2\right]\right. \\
& \left.\left.+\frac{\omega^{2}}{E_{e}^{2}}\right]+(-1) \frac{\vec{\xi}_{e} \cdot \vec{k}_{e}}{E_{e}} \frac{1}{\omega}\left(1+\frac{1}{\beta^{2}} \frac{\omega}{E_{e}}+\frac{1}{2 \beta^{2}} \frac{\omega^{2}}{E_{e}^{2}}\right)\right]\left[\frac{1}{\beta} \ell n\left(\frac{1+\beta}{1-\beta}\right)-2\right]+(-1) \frac{m_{e}}{E_{e}} a_{0} \frac{\vec{\xi}_{e} \cdot \vec{k}_{\bar{\nu}}}{E_{\bar{\nu}}} \frac{1}{\omega}\left(1-\frac{1}{2 \beta^{2}} \frac{\omega^{2}}{E_{e}^{2}}\right) \\
& \times\left[\frac{1}{\beta} \ln \left(\frac{1+\beta}{1-\beta}\right)-2\right]+(-1) \frac{m_{e}}{E_{e}} A_{0} \vec{\xi}_{n} \cdot \vec{\xi}_{e} \frac{1}{\omega}\left(1-\frac{1}{2 \beta^{2}} \frac{\omega^{2}}{E_{e}^{2}}\right)\left[\frac{1}{\beta} \ell n\left(\frac{1+\beta}{1-\beta}\right)-2\right]+(-1) A_{0} \frac{\left.\vec{\xi}_{n} \cdot \vec{k}_{e}\right)\left(\vec{\xi}_{e} \cdot \vec{k}_{e}\right)}{\left(E_{e}+m_{e}\right) E_{e}} \\
& \times\left\{\frac{1}{\omega}\left(1-\frac{1}{2 \beta^{2}} \frac{\omega^{2}}{E_{e}^{2}}\right)\left[\frac{1}{\beta} \ell n\left(\frac{1+\beta}{1-\beta}\right)-2\right]+\left(1+\sqrt{1-\beta^{2}}\right)\left[\frac{1}{\beta^{2}} \frac{\omega}{E_{e}}\left[\frac{1}{\beta} \ell n\left(\frac{1+\beta}{1-\beta}\right)-2\right]+\frac{1}{2 \beta^{2}} \frac{\omega^{2}}{E_{e}^{2}}\left(\frac{3-\beta^{2}}{\beta^{2}}\right.\right.\right. \\
& \left.\left.\left.\times\left[\frac{1}{\beta} \ell n\left(\frac{1+\beta}{1-\beta}\right)-2\right]-2\right)\right]\right\}+(-1) a_{0} \frac{\left(\vec{\xi}_{e} \cdot \vec{k}_{e}\right)\left(\vec{k}_{e} \cdot \vec{k}_{\bar{\nu}}\right)}{\left(E_{e}+m_{e}\right) E_{e} E_{\bar{\nu}}}\left\{\frac{1}{\omega}\left(1-\frac{1}{2 \beta^{2}} \frac{\omega^{2}}{E_{e}^{2}}\right)\left[\frac{1}{\beta} \ell n\left(\frac{1+\beta}{1-\beta}\right)-2\right]+\left(1+\sqrt{1-\beta^{2}}\right)\right. \\
& \left.\times\left[\frac{1}{\beta^{2}} \frac{\omega}{E_{e}}\left[\frac{1}{\beta} \ell n\left(\frac{1+\beta}{1-\beta}\right)-2\right]+\frac{1}{2 \beta^{2}} \frac{\omega^{2}}{E_{e}^{2}}\left(\frac{3-\beta^{2}}{\beta^{2}}\left[\frac{1}{\beta} \ell n\left(\frac{1+\beta}{1-\beta}\right)-2\right]-2\right)\right]\right\}-B_{0} \frac{\left(\vec{\xi}_{n} \cdot \vec{k}_{\bar{\nu}}\right)\left(\vec{\xi}_{e} \cdot \vec{k}_{e}\right)}{E_{e} E_{\bar{\nu}}} \frac{1}{\omega}\left(1+\frac{1}{\beta^{2}} \frac{\omega}{E_{e}}\right. \\
& \left.\left.+\frac{1}{2 \beta^{2}} \frac{\omega^{2}}{E_{e}^{2}}\right)\left[\frac{1}{\beta} \ln \left(\frac{1+\beta}{1-\beta}\right)-2\right]\right\}
\end{aligned}
$$


where $\Phi_{n \gamma}\left(\vec{k}_{e}, \vec{k}_{\bar{\nu}_{e}}, \omega\right)$ is the contribution of the phasevolume of the neutron radiative beta decay [87]

$\Phi_{n \gamma}\left(\vec{k}_{e}, \vec{k}_{\bar{\nu}_{e}}, \omega\right)=1+3 \frac{E_{e}}{m_{N}}\left(1-\frac{\vec{k}_{e} \cdot \vec{k}_{\bar{\nu}_{e}}}{E_{e} E_{\bar{\nu}_{e}}}\right)+O\left(\frac{\omega}{m_{N}}\right)$.

The contribution of the phase-volume Eq. (D6) is the rest of the expression, calculated to NLO in the large nucleon mass
$m_{N}$ expansion and the integration over the directions of the photon 3-momentum [87]. As has been shown in [87], the contributions of the terms $O\left(\omega / m_{N}\right)$ are of order of $10^{-6}$ and even smaller. So, the $O\left(\alpha E_{e} / m_{N}\right)$ corrections can be induced only by the second term in Eq. (D6).

Having integrated over $\omega$ we arrive at the following expression:

$$
\begin{aligned}
\frac{d^{6} \lambda_{\beta \gamma}\left(E_{e}, \vec{k}_{e}, \vec{k}_{\bar{\nu}}, \vec{\xi}_{n}, \vec{\xi}_{e}\right)}{d E_{e} d \Omega_{e} d \Omega_{\bar{\nu}}}= & \left(1+3 g_{A}^{2}\right) \frac{\alpha\left|G_{V}\right|^{2}}{\pi} \frac{\sqrt{E_{e}}-m_{e}^{2}}{16 \pi^{5}} E_{e} F\left(E_{e}, Z=1\right)\left(E_{0}-E_{e}\right)^{2}\left[1+3 \frac{E_{e}}{m_{N}}\left(1-\frac{\vec{k}_{e} \cdot \vec{k}_{\bar{\nu}_{e}}}{E_{e} E_{\bar{\nu}_{e}}}\right)\right] \\
& \times\left\{g_{\beta \gamma}^{(1)}\left(E_{e}, \mu\right)+a_{0} \frac{\vec{k}_{e} \cdot \vec{k}_{\bar{\nu}}}{E_{e} E_{\bar{\nu}}} g_{\beta \gamma}^{(2)}\left(E_{e}, \mu\right)+A_{0} g_{\beta \gamma}^{(2)}\left(E_{e}, \mu\right)+B_{0} \frac{\vec{\xi}_{n} \cdot \vec{k}_{\bar{\nu}}}{E_{\bar{\nu}}} g_{\beta \gamma}^{(1)}\left(E_{e}, \mu\right)+(-1) \frac{\vec{\xi}_{e} \cdot \vec{k}_{e}}{E_{e}} g_{\beta \gamma}^{(2)}\left(E_{e}, \mu\right)\right. \\
& +(-1) \frac{m_{e}}{E_{e}} a_{0} \frac{\vec{\xi}_{e} \cdot \vec{k}_{\bar{\nu}}}{E_{\bar{\nu}}} g_{\beta \gamma}^{(3)}\left(E_{e}, \mu\right)+(-1) \frac{m_{e}}{E_{e}} A_{0} \vec{\xi}_{n} \cdot \vec{\xi}_{e} g_{\beta \gamma}^{(3)}\left(E_{e}, \mu\right)+(-1) A_{0} \frac{\left.\vec{\xi}_{n} \cdot \vec{k}_{e}\right)\left(\vec{\xi}_{e} \cdot \vec{k}_{e}\right)}{\left(E_{e}+m_{e}\right) E_{e}} g_{\beta \gamma}^{(4)}\left(E_{e}, \mu\right) \\
& \left.+(-1) a_{0} \frac{\left(\vec{\xi}_{e} \cdot \vec{k}_{e}\right)\left(\vec{k}_{e} \cdot \vec{k}_{\bar{\nu}}\right)}{\left(E_{e}+m_{e}\right) E_{e} E_{\bar{\nu}}} g_{\beta \gamma}^{(4)}\left(E_{e}, \mu\right)-B_{0} \frac{\left(\vec{\xi}_{n} \cdot \vec{k}_{\bar{\nu}}\right)\left(\vec{\xi}_{e} \cdot \vec{k}_{e}\right)}{E_{e} E_{\bar{\nu}}} g_{\beta \gamma}^{(2)}\left(E_{e}, \mu\right)\right\} .
\end{aligned}
$$

The functions $g_{\beta \gamma}^{(j)}\left(E_{e}, \mu\right)$ for $j=1,2,3,4$ have been calculated in [11,12,14,29]. As has been shown in [11,12,14,29], the difference between functions $g_{\beta \gamma}^{(1)}\left(E_{e}, \mu\right)-g_{\beta \gamma}^{(j)}\left(E_{e}, \mu\right)$ for $j=2,3,4$ does not depend on the regularization, i.e.,

$$
\lim _{\mu \rightarrow 0}\left(g_{\beta \gamma}^{(1)}\left(E_{e}, \mu\right)-g_{\beta \gamma}^{(j)}\left(E_{e}, \mu\right)\right)=\lim _{\omega_{\min } \rightarrow 0}\left(g_{\beta \gamma}^{(1)}\left(E_{e}, \omega_{\min }\right)-g_{\beta \gamma}^{(j)}\left(E_{e}, \omega_{\min }\right)\right)
$$

where $\omega_{\min }$ is a noncovariant infrared cutoff, which can also be treated as the photon-energy threshold of the detector $[11,12,14,29]$. Summing up Eqs. (D4) and (D7) we obtain the total electron-energy and angular distribution of the neutron beta decay
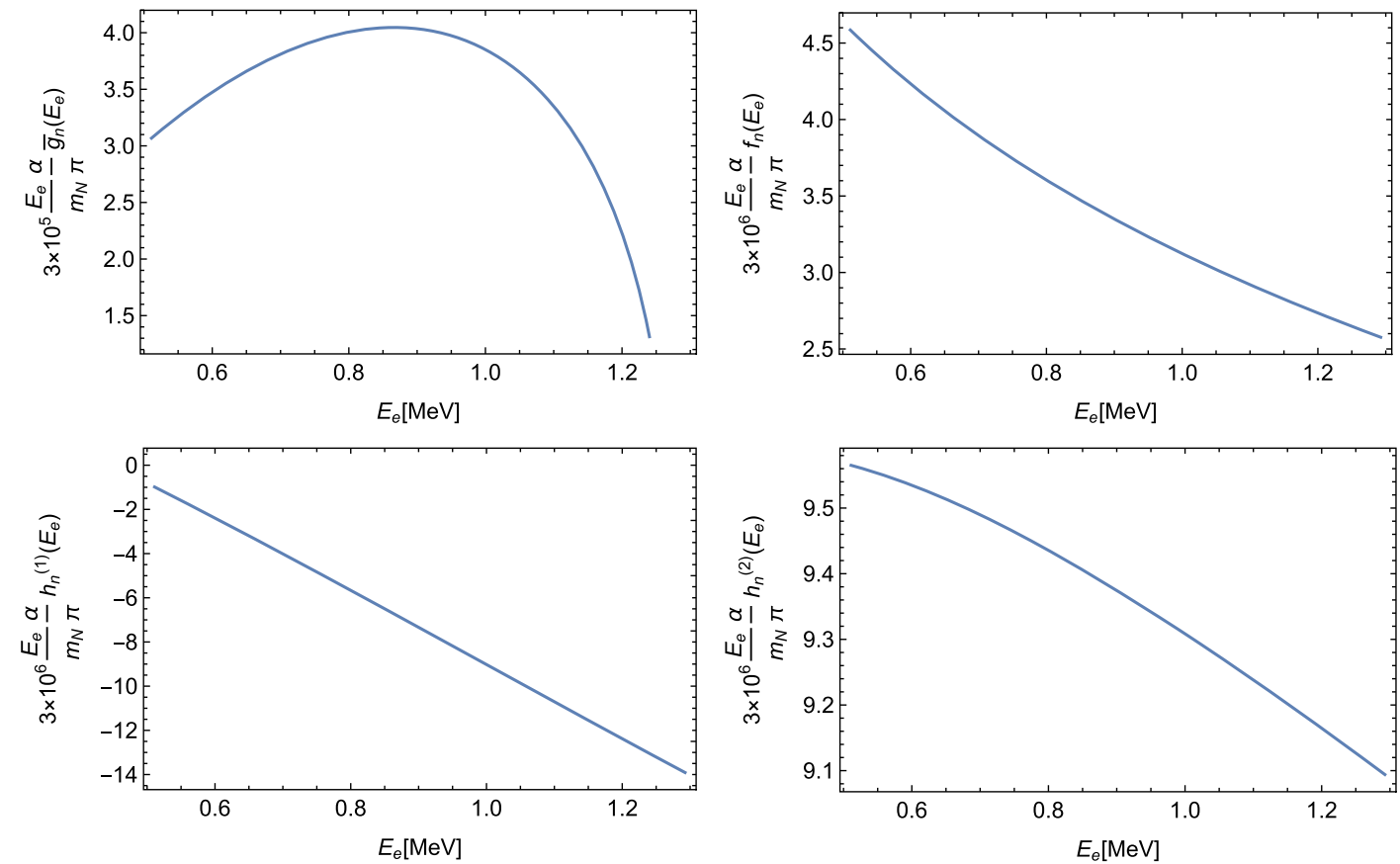

FIG. 1. The outer radiative corrections of order $O\left(\alpha E_{e} / m_{N}\right)$ in the electron-energy region $m_{e} \leq E_{e}<E_{0}$, induced by the outer radiative corrections of order $O(\alpha / \pi)$ and the phase-volume of the neutron beta decay, calculated to NLO in the large nucleon mass $m_{N}$ expansion. 


$$
\begin{aligned}
& \frac{d^{5} \lambda_{n}\left(E_{e}, \vec{k}_{e}, \vec{k}_{\bar{\nu}}, \vec{\xi}_{n}, \vec{\xi}_{e}\right)}{d E_{e} d \Omega_{e} d \Omega_{\bar{\nu}}} \\
& =\left(1+3 g_{A}^{2}\right) \frac{\left|G_{V}\right|^{2}}{16 \pi^{5}}\left(E_{0}-E_{e}\right)^{2} \sqrt{E_{e}^{2}-m_{e}^{2}} E_{e} F\left(E_{e}, Z=1\right)\left[1+3 \frac{E_{e}}{m_{N}}\left(1-\frac{\vec{k}_{e} \cdot \vec{k}_{\bar{\nu}}}{E_{e} E_{\bar{\nu}}}\right)\right] \\
& \quad \times\left\{\left(1+\frac{\alpha}{\pi} \bar{g}_{n}\left(E_{e}\right)\right)+a_{0}\left[1+\frac{\alpha}{\pi}\left(\bar{g}_{n}\left(E_{e}\right)+f_{n}\left(E_{e}\right)\right)\right] \frac{\vec{k}_{e} \cdot \vec{k}_{\bar{\nu}}}{E_{e} E_{\bar{\nu}}}+A_{0}\left[1+\frac{\alpha}{\pi}\left(\bar{g}_{n}\left(E_{e}\right)+f_{n}\left(E_{e}\right)\right)\right] \frac{\vec{\xi}_{n} \cdot \vec{k}_{e}}{E_{e}}\right. \\
& \quad+B_{0}\left(1+\frac{\alpha}{\pi} \bar{g}_{n}\left(E_{e}\right)\right) \frac{\vec{\xi}_{n} \cdot \vec{k}_{\bar{\nu}}}{E_{\bar{\nu}}}\left[1+\frac{\alpha}{\pi}\left(\bar{g}_{n}\left(E_{e}\right)+f_{n}\left(E_{e}\right)\right)\right] \frac{\vec{\xi}_{e} \cdot \vec{k}_{e}}{E_{e}}-\frac{m_{e}}{E_{e}} a_{0}\left[1+\frac{\alpha}{\pi}\left(\bar{g}_{n}\left(E_{e}\right)+h_{n}^{(1)}\left(E_{e}\right)\right)\right] \frac{\vec{\xi}_{e} \cdot \vec{k}_{\bar{\nu}}}{E_{\bar{\nu}}} \\
& \quad-\frac{m_{e}}{E_{e}} A_{0}\left[1+\frac{\alpha}{\pi}\left(\bar{g}_{n}\left(E_{e}\right)+h_{n}^{(1)}\left(E_{e}\right)\right] \vec{\xi}_{n} \cdot \vec{\xi}_{e}-A_{0}\left[1+\frac{\alpha}{\pi}\left(\vec{g}_{n}\left(E_{e}\right)+h_{n}^{(2)}\left(E_{e}\right)\right] \frac{\left(\vec{\xi}_{n} \cdot \vec{k}_{e}\right)\left(\vec{k}_{e} \cdot \vec{\xi}_{e}\right)}{\left(E_{e}+m_{e}\right) E_{e}}\right.\right. \\
& \left.\quad-a_{0}\left[1+\frac{\alpha}{\pi}\left(\bar{g}_{n}\left(E_{e}\right)+h_{n}^{(2)}\left(E_{e}\right)\right)\right] \frac{\left(\vec{\xi}_{e} \cdot \vec{k}_{e}\right)\left(\vec{k}_{e} \cdot \vec{k}_{\bar{\nu}}\right)}{\left(E_{e}+m_{e}\right) E_{e} E_{\bar{\nu}}}-B_{0}\left[1+\frac{\alpha}{\pi}\left(\vec{g}_{n}\left(E_{e}\right)+f_{n}\left(E_{e}\right)\right)\right] \frac{\left(\vec{\xi}_{n} \cdot \vec{k}_{\bar{\nu}}\right)\left(\vec{\xi}_{e} \cdot \vec{k}_{e}\right)}{E_{e} E_{\bar{\nu}}}\right\} .
\end{aligned}
$$

The detailed calculation of the functions $(\alpha / \pi) \bar{g}_{n}\left(E_{e}\right)$ [19], $(\alpha / \pi) f_{n}\left(E_{e}\right) \quad[21], \quad(\alpha / \pi) h_{n}^{(1)}\left(E_{e}\right), \quad$ and $(\alpha / \pi) h_{n}^{(2)}\left(E_{e}\right)$ one can find in $[11,12,14,29,30]$. The behavior of the functions $(\alpha / \pi) \bar{g}_{n}\left(E_{e}\right),(\alpha / \pi) f_{n}\left(E_{e}\right),(\alpha / \pi) h_{n}^{(1)}\left(E_{e}\right)$, and $(\alpha / \pi) h_{n}^{(2)}\left(E_{e}\right)$ multiplied by the factor $3\left(E_{e} / m_{N}\right)$, caused by the phase-volume of the neutron beta decay, is shown in Fig. 1.

One can see that in the electron-energy region $m_{e} \leq E_{e}<E_{0}$ the functions $3(\alpha / \pi)\left(E_{e} / m_{N}\right) \bar{g}_{n}\left(E_{e}\right)$, $3(\alpha / \pi)\left(E_{e} / m_{N}\right) h_{n}^{(1)}\left(E_{e}\right)$, and $3(\alpha / \pi)\left(E_{e} / m_{N}\right) h_{n}^{(2)}\left(E_{e}\right)$ are of order of a few parts of $10^{-5}$, whereas the function $3(\alpha / \pi)\left(E_{e} / m_{N}\right) f_{n}\left(E_{e}\right)$ is of order of a few parts of $10^{-6}$. However, the functions $3(\alpha / \pi)\left(E_{e} / m_{N}\right) h_{n}^{(j)}\left(E_{e}\right)$ for $j=1$, 2 are multiplied by either $\left(m_{e} / E_{e}\right) X_{0}$ or $X_{0}$, where $X_{0}=a_{0}, A_{0} \sim-0.1$. As a result, the values of the functions $3(\alpha / \pi)\left(m_{e} / m_{N}\right) X_{0} h_{n}^{(j)}\left(E_{e}\right)$ and $3(\alpha / \pi)\left(E_{e} / m_{N}\right) \times$ $X_{0} h_{n}^{(j)}\left(E_{e}\right)$ become of order of a few parts of $10^{-6}$. Hence, the outer radiative corrections of order $O\left(\alpha E_{e} / m_{N}\right)$ are defined by the function $3(\alpha / \pi)\left(E_{e} / m_{N}\right) \bar{g}_{n}\left(E_{e}\right)$ only.

As a result, the correlation function $\zeta\left(E_{e}\right)$ and the correlation coefficients $a\left(E_{e}\right), Q_{n}\left(E_{e}\right), Q_{e}\left(E_{e}\right)$, and $K_{e}\left(E_{e}\right)$ acquire the following outer or model-independent $O\left(\alpha E_{e} / m_{N}\right)$ radiative corrections:

$$
\begin{aligned}
\zeta\left(E_{e}\right)_{\mathrm{RC}-\mathrm{PhV}} & =3 \frac{\alpha}{\pi} \frac{E_{e}}{m_{N}} \bar{g}_{n}\left(E_{e}\right), \\
a\left(E_{e}\right)_{\mathrm{RC}-\mathrm{PhV}} & =-3 \frac{\alpha}{\pi} \frac{E_{e}}{m_{N}} \bar{g}_{n}\left(E_{e}\right), \\
Q_{n}\left(E_{e}\right)_{\mathrm{RC}-\mathrm{PhV}} & =-3 B_{0} \frac{\alpha}{\pi} \frac{E_{e}}{m_{N}} \bar{g}_{n}\left(E_{e}\right), \\
Q_{e}\left(E_{e}\right)_{\mathrm{RC}-\mathrm{PhV}} & =B_{0} \frac{\alpha}{\pi} \frac{E_{e}}{m_{N}} \bar{g}_{n}\left(1+\frac{m}{E_{e}}\right), \\
K_{e}\left(E_{e}\right)_{\mathrm{RC}-\mathrm{PhV}} & =3 \frac{\alpha}{\pi} \frac{E_{e}}{m_{N}} \bar{g}_{n}\left(E_{e}\right)\left(1+\frac{m_{e}}{E_{e}}\right) .
\end{aligned}
$$

These radiative corrections are of order of a few parts of $10^{-5}$ in the experimental electron-energy region $0.811 \mathrm{MeV} \leq E_{e} \leq 1.211 \mathrm{MeV}$ [5]. They are plotted in [46]. The analytical expression of the function $\bar{g}_{n}\left(E_{e}\right)$ is given in Eq. (A19). We have to notice that there is the contribution, proportional to $3(\alpha / \pi)\left(E_{e} / m_{N}\right) \bar{g}_{n}\left(E_{e}\right)$, to the electron-energy and angular distribution of the neutron beta decay with the correlation structure beyond the standard correlation structures in Eq. (1) [see the last term in Eq. (F2)].

\section{APPENDIX E: ANALYTICAL EXPRESSIONS FOR THE CORRELATION FUNCTION $\zeta\left(E_{e}\right)$ AND CORRELATION COEFFICIENTS $a\left(E_{e}\right), A\left(E_{e}\right)$, $B\left(E_{e}\right), K_{n}\left(E_{e}\right), Q_{n}\left(E_{e}\right)$, AND $A^{(\beta)}\left(E_{e}\right)$}

In this Appendix we give the analytical expressions for the correlation function $\zeta\left(E_{e}\right)$ and the correlation coefficients $X\left(E_{e}\right)$ for $X=a, A, B, \ldots, U$ and also for the correlation coefficient $A^{(\beta)}\left(E_{e}\right)=A\left(E_{e}\right)+\frac{1}{3} Q_{n}\left(E_{e}\right)$ as functions of the electron energy $E_{e}$ and the axial coupling constant $g_{A}$. The correlation function $\zeta\left(E_{e}\right)$ and correlation coefficients are calculated with a theoretical accuracy of about $10^{-6}$.

For the correlation function $\zeta\left(E_{e}\right)$ we obtain the following expression:

$$
\begin{aligned}
\zeta\left(E_{e}\right)= & 1+\frac{\alpha}{\pi} \bar{g}_{n}\left(E_{e}\right)+\frac{1}{1+3 g_{A}^{2}} \frac{E_{0}}{m_{N}}\left[-2 g_{A}\left(g_{A}+(\kappa+1)\right)\right. \\
& +\left(10 g_{A}^{2}+4(\kappa+1) g_{A}+2\right) \frac{E_{e}}{E_{0}} \\
& \left.-2 g_{A}\left(g_{A}+(\kappa+1)\right) \frac{m_{e}^{2}}{E_{0}^{2}} \frac{E_{0}}{E_{e}}\right]+\zeta\left(E_{e}\right)_{\mathrm{RC}-\mathrm{NLO}} \\
& +\zeta\left(E_{e}\right)_{\mathrm{RC}-\mathrm{PhV}}+\zeta\left(E_{e}\right)_{\mathrm{N}^{2} \mathrm{~L} 0}+\zeta\left(E_{e}\right)_{\mathrm{WC}} . \quad(\mathrm{E} 1)
\end{aligned}
$$


Here $\bar{g}_{n}\left(E_{e}\right)$ is Sirlin's function [19] [see also Eq. (A19)]. Then, the term proportional to $E_{0} / m_{N}$ defines the wellknown corrections $O\left(E_{e} / m_{N}\right)$, caused by weak magnetism and proton recoil (see, for example, [11,23]). The corrections $\zeta\left(E_{e}\right)_{\mathrm{RC}-\mathrm{NLO}}, \zeta\left(E_{e}\right)_{\mathrm{RC}-\mathrm{PhV}}, \zeta\left(E_{e}\right)_{\mathrm{N}^{2} \mathrm{~L} 0}$, and $\zeta\left(E_{e}\right)_{\mathrm{WC}}$ are defined by the $O\left(\alpha E_{e} / m_{N}\right)$ inner and outer radiative corrections, the $O\left(E_{e}^{2} / m_{N}^{2}\right)$ corrections, caused by weak magnetism and proton recoil, and Wilkinson's corrections, respectively. They are equal to

$$
\begin{aligned}
\zeta\left(E_{e}\right)_{\mathrm{RC}-\mathrm{NLO}}= & \frac{1}{1+3 g_{A}^{2}} \frac{\alpha}{\pi} \frac{E_{e}}{m_{N}}\left(f_{V}\left(E_{e}\right)+\sqrt{1-\beta^{2}} f_{S}\left(E_{e}\right)+g_{A} g_{V}\left(E_{e}\right) \beta^{2}+\left(1+2 g_{A}\right) h_{A}\left(E_{e}\right) \beta^{2}\right. \\
& \left.+g_{A} \frac{E_{0}-E_{e}}{E_{e}} \sqrt{1-\beta^{2}} h_{S}\left(E_{e}\right)+3 g_{A}^{2} f_{A}\left(E_{e}\right)+3 g_{A}^{2} \sqrt{1-\beta^{2}} f_{T}\left(E_{e}\right)\right)+\frac{3 g_{A}^{2}}{1+3 g_{A}^{2}} \frac{\alpha}{\pi} \frac{5}{2} \frac{m_{N}^{2}}{M_{W}^{2}} \ell n \frac{M_{W}^{2}}{m_{N}^{2}} \\
& +\frac{1}{1+3 g_{A}^{2}} \frac{\alpha}{\pi}\left(\bar{g}_{\mathrm{st}}\left(E_{e}\right)+3 g_{A} \bar{f}_{\mathrm{st}}\left(E_{e}\right)\right), \\
\zeta\left(E_{e}\right)_{\mathrm{RC}-\mathrm{PhV}}= & 3 \frac{\alpha}{\pi} \frac{E_{e}}{m_{N}} \bar{g}_{n}\left(E_{e}\right), \\
\zeta\left(E_{e}\right)_{\mathrm{N}^{2} \mathrm{~L} 0}= & 6 \frac{E_{e}^{2}}{m_{N}^{2}}\left\{\left(1-\frac{1}{4} \frac{E_{0}}{E_{e}}\right)+\frac{1}{3}\left[1-2 a_{0}\left(1-\frac{1}{8} \frac{E_{0}}{E_{e}}\right)\right]\left(1-\frac{m_{e}^{2}}{E_{e}^{2}}\right)\right\}+3 \frac{E_{e}}{m_{N}} \zeta\left(E_{e}\right)_{\mathrm{NLO}}-\frac{E_{e}}{m_{N}} \bar{a}\left(E_{e}\right)_{\mathrm{NLO}} \\
& \times\left(1-\frac{m_{e}^{2}}{E_{e}^{2}}\right)-\frac{8}{1+3 g_{A}^{2}}\left(\frac{E_{0}^{2}}{M_{V}^{2}}+3 g_{A}^{2} \frac{E_{0}^{2}}{M_{A}^{2}}\right) \frac{E_{e}}{E_{0}}\left(1-\frac{E_{e}}{E_{0}}\right), \\
\zeta\left(E_{e}\right)_{\mathrm{WC}}= & 3.76 \times 10^{-4}-5.26 \times 10^{-5} \frac{E_{e}}{E_{0}}-1.26 \times 10^{-5} \frac{E_{e}^{2}}{E_{0}^{2}}-\frac{\pi \alpha}{\beta} \frac{E_{e}}{m_{N}} .
\end{aligned}
$$

The terms, proportional to $E_{0}^{2} / M_{V}^{2}$ and $E_{0}^{2} / M_{A}^{2}$, appear from the contributions of the vector and axial-vector form factors of the neutron beta decay. For numerical analysis we use $M_{V}=813 \mathrm{MeV}$ and $M_{A}=1077 \mathrm{MeV}$ [24]. The first three terms in $\zeta\left(E_{e}\right)_{\mathrm{WC}}$ do not depend practically on the axial coupling constant $g_{A}$.

Of course, the correlation function $\zeta\left(E_{e}\right)$ in Eq. (E1) should be supplemented by the inner radiative corrections $\Delta_{R}^{V}$ and $\Delta_{R}^{A}$ of order $O(\alpha / \pi)$, caused by the Feynman $\gamma W^{-}$-box diagrams and calculated in [48-56].

For the correlation coefficient $a\left(E_{e}\right)$ we obtain the following expression:

$$
\begin{aligned}
a\left(E_{e}\right)= & a_{0}\left\{1+\frac{\alpha}{\pi} f_{n}\left(E_{e}\right)+\frac{1}{\left(1-g_{A}^{2}\right)\left(1+3 g_{A}^{2}\right)} \frac{E_{0}}{m_{N}}\left(a_{1}+a_{2} \frac{E_{e}}{E_{0}}+a_{3} \frac{E_{0}}{E_{e}}\right)\right\} \\
& +a\left(E_{e}\right)_{\mathrm{RC}-\mathrm{NLO}}+a\left(E_{e}\right)_{\mathrm{RC}-\mathrm{PhV}}+a\left(E_{e}\right)_{\mathrm{N}^{2} \mathrm{LO}}+a\left(E_{e}\right)_{\mathrm{WC}},
\end{aligned}
$$

where the function $f_{n}\left(E_{e}\right)$ has been calculated by Shann [21] [see also Eq. (A19) and [11,12] ] and the coefficients $a_{1}, a_{2}$, and $a_{3}$ are equal to [11]

$$
\begin{aligned}
& a_{1}=4 g_{A}\left(g_{A}^{2}+1\right)\left(g_{A}+(\kappa+1)\right), \\
& a_{2}=-26 g_{A}^{4}-8(\kappa+1) g_{A}^{3}-20 g_{A}^{2}-8(\kappa+1) g_{A}-2, \\
& a_{3}=-2 g_{A}\left(g_{A}^{2}-1\right)\left(g_{A}+(\kappa+1)\right) \frac{m_{e}^{2}}{E_{0}^{2}} .
\end{aligned}
$$

The corrections $a\left(E_{e}\right)_{\mathrm{RC}-\mathrm{NLO}}, a\left(E_{e}\right)_{\mathrm{RC}-\mathrm{PhV}}, a\left(E_{e}\right)_{\mathrm{N}^{2} \mathrm{~L} 0}$, and $a\left(E_{e}\right)_{\mathrm{WC}}$, defined by the $O\left(\alpha E_{e} / m_{N}\right)$ inner and outer radiative corrections, the $O\left(E_{e}^{2} / m_{N}^{2}\right)$ corrections, caused by weak magnetism and proton recoil, and Wilkinson's corrections, respectively, are given by 


$$
\begin{aligned}
a\left(E_{e}\right)_{\mathrm{RC}-\mathrm{NLO}}= & \frac{1}{1+3 g_{A}^{2}} \frac{\alpha}{\pi} \frac{E_{e}}{m_{N}}\left(f_{V}\left(E_{e}\right)+g_{A} \sqrt{1-\beta^{2}} g_{S}\left(E_{e}\right)+g_{A} g_{V}\left(E_{e}\right)+\left(1-2 g_{A}\right) h_{A}\left(E_{e}\right)-g_{A}^{2} f_{A}\left(E_{e}\right)\right) \\
& -\frac{g_{A}^{2}}{1+3 g_{A}^{2}} \frac{\alpha}{\pi} \frac{5}{2} \frac{m_{N}^{2}}{M_{W}^{2}} \ell n \frac{M_{W}^{2}}{m_{N}^{2}}+\frac{1}{1+3 g_{A}^{2}} \frac{\alpha}{\pi}\left(\bar{g}_{\mathrm{st}}\left(E_{e}\right)-g_{A} \bar{f}_{\mathrm{st}}\left(E_{e}\right)\right)-a_{0} \zeta\left(E_{e}\right)_{\mathrm{RC}-\mathrm{NLO}}, \\
a\left(E_{e}\right)_{\mathrm{RC}-\mathrm{PhV}}= & -3 \frac{\alpha}{\pi} \frac{E_{e}}{m_{N}} \bar{g}_{n}\left(E_{e}\right), \\
a\left(E_{e}\right)_{\mathrm{N}{ }^{2} \mathrm{~L} 0}= & 12 \frac{E_{e}^{2}}{m_{N}^{2}}\left\{-\left(1-\frac{1}{8} \frac{E_{0}}{E_{e}}\right)+\frac{1}{3} a_{0}\left(1-\frac{1}{4} \frac{E_{0}}{E_{e}}\right)\right\}+3 \frac{E_{e}}{m_{N}} \bar{a}\left(E_{e}\right)_{\mathrm{NLO}}-\frac{E_{e}}{m_{N}} \zeta\left(E_{e}\right)_{\mathrm{NLO}} \\
& -a\left(E_{e}\right)_{\mathrm{NLO}} \zeta\left(E_{e}\right)_{\mathrm{NLO}}+\frac{8}{1+3 g_{A}^{2}}\left(\frac{E_{0}^{2}}{M_{V}^{2}}+3 g_{A}^{2} \frac{E_{0}^{2}}{M_{A}^{2}}\right) \frac{E_{e}}{E_{0}}\left(1-\frac{E_{e}}{E_{0}}\right), \\
a\left(E_{e}\right)_{\mathrm{WC}}= & -3.76 \times 10^{-4} a_{0}-\frac{\alpha \pi}{\beta^{3}} \frac{E_{0}-E_{e}}{m_{N}} .
\end{aligned}
$$

For the correlation coefficient $A\left(E_{e}\right)$ we obtain the following expression:

$$
\begin{aligned}
A\left(E_{e}\right)= & A_{0}\left\{1+\frac{\alpha}{\pi} f_{n}\left(E_{e}\right)+\frac{1}{2 g_{A}\left(1-g_{A}\right)\left(1+3 g_{A}^{2}\right)} \frac{E_{0}}{m_{N}}\left(A_{1}+A_{2} \frac{E_{e}}{E_{0}}+A_{3} \frac{E_{0}}{E_{e}}\right)\right\} \\
& +A\left(E_{e}\right)_{\mathrm{RC}-\mathrm{NLO}}+A\left(E_{e}\right)_{\mathrm{N}^{2} \mathrm{LO}}+A\left(E_{e}\right)_{\mathrm{WC}}
\end{aligned}
$$

where the function $f_{n}\left(E_{e}\right)$ has been calculated by Shann [21] [see also Eq. (A19)] and the coefficients $A_{1}, A_{2}$, and $A_{3}$ are given by [11]

$$
\begin{aligned}
& A_{1}=-g_{A}^{4}-\kappa g_{A}^{3}+(\kappa+2) g_{A}^{2}+\kappa g_{A}-(\kappa+1), \\
& A_{2}=5 g_{A}^{4}-\kappa g_{A}^{3}-(5 \kappa+6) g_{A}^{2}-3 \kappa g_{A}+(\kappa+1), \\
& A_{3}=-4 g_{A}^{2}\left(g_{A}-1\right)\left(g_{A}+(\kappa+1)\right) \frac{m_{e}^{2}}{E_{0}^{2}}
\end{aligned}
$$

The corrections $A\left(E_{e}\right)_{\mathrm{RC}-\mathrm{NLO}}, A\left(E_{e}\right)_{\mathrm{N}^{2} \mathrm{~L} 0}$, and $A\left(E_{e}\right)_{\mathrm{WC}}$, defined by the $O\left(\alpha E_{e} / m_{N}\right)$ inner radiative corrections, the $O\left(E_{e}^{2} / m_{N}^{2}\right)$ corrections, caused by weak magnetism and proton recoil, and Wilkinson's corrections, respectively, are equal to

$$
\begin{aligned}
A\left(E_{e}\right)_{\mathrm{RC}-\mathrm{NLO}}= & \frac{1}{1+3 g_{A}^{2}} \frac{\alpha}{\pi} \frac{E_{e}}{m_{N}}\left(g_{A} f_{V}\left(E_{e}\right)+\sqrt{1-\beta^{2}} g_{S}\left(E_{e}\right)+g_{V}\left(E_{e}\right)-g_{A} h_{A}\left(E_{e}\right)+g_{A}\left(1-2 g_{A}\right) f_{A}\left(E_{e}\right)\right) \\
& +\frac{1}{1+3 g_{A}^{2}} g_{A}\left(1-2 g_{A}\right) \frac{\alpha}{\pi} \frac{5}{2} \frac{m_{N}^{2}}{M_{W}^{2}} \ln \frac{M_{W}^{2}}{m_{N}^{2}}+\frac{1}{1+3 g_{A}^{2}} \frac{\alpha}{\pi}\left(g_{A} \bar{g}_{\mathrm{st}}\left(E_{e}\right)+\left(1-2 g_{A}\right) \bar{f}_{\mathrm{st}}\left(E_{e}\right)\right)-A_{0} \zeta\left(E_{e}\right)_{\mathrm{RC}-\mathrm{NLO}} \\
A\left(E_{e}\right)_{\mathrm{N} 2 \mathrm{~L} 0}= & 3 \frac{E_{e}}{m_{N}} \bar{A}\left(E_{e}\right)_{\mathrm{NLO}}-\frac{E_{e}}{m_{N}} \bar{K}_{n}\left(E_{e}\right)_{\mathrm{NLO}}\left(1-\frac{m_{e}^{2}}{E_{e}^{2}}\right)-A\left(E_{e}\right)_{\mathrm{NLO}} \zeta\left(E_{e}\right)_{\mathrm{NLO}} \\
A\left(E_{e}\right)_{\mathrm{WC}}= & -3.76 \times 10^{-4} A_{0} .
\end{aligned}
$$

For the correlation coefficient $B\left(E_{e}\right)$ we obtain the following expression:

$$
\begin{aligned}
B\left(E_{e}\right)= & B_{0}\left\{1+\frac{1}{2 g_{A}\left(1+g_{A}\right)\left(1+3 g_{A}^{2}\right)} \frac{E_{0}}{m_{N}}\left(B_{1}+B_{2} \frac{E_{e}}{E_{0}}+B_{3} \frac{E_{0}}{E_{e}}\right)\right\} \\
& +B\left(E_{e}\right)_{\mathrm{RC}-\mathrm{NLO}}+B\left(E_{e}\right)_{\mathrm{N}^{2} \mathrm{LO}}+B\left(E_{e}\right)_{\mathrm{WC}},
\end{aligned}
$$

where the coefficients $B_{1}, B_{2}$, and $B_{3}$ are given by [11] 


$$
\begin{aligned}
& B_{1}=-2 g_{A}\left(1-g_{A}\right)^{2}\left(g_{A}+(\kappa+1)\right), \\
& B_{2}=g_{A}^{4}+(\kappa-4) g_{A}^{3}-(5 \kappa+2) g_{A}^{2}+(3 \kappa+4) g_{A}+(\kappa+1), \\
& B_{3}=\left(g_{A}^{2}-1\right)\left(1+g_{A}\right)\left(g_{A}+(\kappa+1)\right) \frac{m_{e}^{2}}{E_{0}^{2}} .
\end{aligned}
$$

The corrections $B\left(E_{e}\right)_{\mathrm{RC}-\mathrm{NLO}}, B\left(E_{e}\right)_{\mathrm{N}^{2} \mathrm{~L} 0}$, and $B\left(E_{e}\right)_{\mathrm{WC}}$, defined by the $O\left(\alpha E_{e} / m_{N}\right)$ inner radiative corrections, the $O\left(E_{e}^{2} / m_{N}^{2}\right)$ corrections, caused by weak magnetism and proton recoil, and Wilkinson's corrections, respectively, are equal to

$$
\begin{aligned}
B\left(E_{e}\right)_{\mathrm{RC}-\mathrm{NLO}}= & \frac{1}{1+3 g_{A}^{2}} \frac{\alpha}{\pi} \frac{E_{e}}{m_{N}}\left(g_{A} f_{V}\left(E_{e}\right)+g_{A} \sqrt{1-\beta^{2}} f_{S}\left(E_{e}\right)+\frac{E_{0}-E_{e}}{E_{e}} \sqrt{1-\beta^{2}} h_{S}\left(E_{e}\right)+g_{A} g_{V}\left(E_{e}\right) \beta^{2}\right. \\
& \left.-\left(1-2 g_{A}\right) h_{A}\left(E_{e}\right) \beta^{2}+g_{A}\left(1+2 g_{A}\right) f_{A}\left(E_{e}\right)+g_{A}\left(1+2 g_{A}\right) \sqrt{1-\beta^{2}} f_{T}\left(E_{e}\right)\right)+\frac{1}{1+3 g_{A}^{2}} g_{A}\left(1+2 g_{A}\right) \\
& \times \frac{\alpha}{\pi} \frac{5}{2} \frac{m_{N}^{2}}{M_{W}^{2}} \ell n \frac{M_{W}^{2}}{m_{N}^{2}}+\frac{1}{1+3 g_{A}^{2}} \frac{\alpha}{\pi}\left(g_{A} \bar{g}_{\mathrm{st}}\left(E_{e}\right)+\left(1+2 g_{A}\right) \bar{f}_{\mathrm{st}}\left(E_{e}\right)\right)-B_{0} \zeta\left(E_{e}\right)_{\mathrm{RC}-\mathrm{NLO}} \\
B\left(E_{e}\right)_{\mathrm{N}^{2} \mathrm{~L} 0}= & 6 \frac{E_{e}^{2}}{m_{N}^{2}} B_{0}\left(1-\frac{1}{4} \frac{E_{0}}{E_{e}}\right)+3 \frac{E_{e}}{m_{N}} \bar{B}\left(E_{e}\right)_{\mathrm{NLO}}-B\left(E_{e}\right)_{\mathrm{NLO}} \zeta\left(E_{e}\right)_{\mathrm{NLO}}-B_{0} \zeta\left(E_{e}\right)_{\mathrm{N}^{2} \mathrm{LO}} \\
& -\frac{8 g_{A}}{1+3 g_{A}^{2}}\left(\frac{E_{0}^{2}}{M_{V}^{2}}+\left(1+2 g_{A}\right) \frac{E_{0}^{2}}{M_{A}^{2}}\right) \frac{E_{e}}{E_{0}}\left(1-\frac{E_{e}}{E_{0}}\right) \\
B\left(E_{e}\right)_{\mathrm{WC}}= & B_{0}\left(3.76 \times 10^{-4}-5.26 \times 10^{-5} \frac{E_{e}}{E_{0}}-1.26 \times 10^{-5} \frac{E_{e}^{2}}{E_{0}^{2}}\right)+\frac{1}{3} a_{0} B_{0} \frac{\pi \alpha}{\beta} \frac{E_{0}-E_{e}}{m_{N}} .
\end{aligned}
$$

The coefficients of Wilkinson's term in the parentheses do not practically depend on the axial coupling constant $g_{A}$.

For the correlation coefficient $K_{n}\left(E_{e}\right)$ we obtain the following expressions:

$$
K_{n}\left(E_{e}\right)=\frac{1}{1+3 g_{A}^{2}} \frac{E_{e}}{m_{N}}\left(5 g_{A}^{2}+(\kappa-4) g_{A}-(\kappa+1)\right)+K_{n}\left(E_{e}\right)_{\mathrm{RC}}+K_{n}\left(E_{e}\right)_{\mathrm{N}^{2} \mathrm{LO}}+K_{n}\left(E_{e}\right)_{\mathrm{WC}}
$$

The corrections $K_{n}\left(E_{e}\right)_{\mathrm{RC}-\mathrm{NLO}}, K_{n}\left(E_{e}\right)_{\mathrm{N}^{2} \mathrm{~L} 0}$, and $K_{n}\left(E_{e}\right)_{\mathrm{WC}}$, defined by the $O\left(\alpha E_{e} / m_{N}\right)$ inner radiative corrections, the $O\left(E_{e}^{2} / m_{N}^{2}\right)$ corrections, caused by weak magnetism and proton recoil, and Wilkinson's corrections, respectively, are equal to

$$
\begin{aligned}
K_{n}\left(E_{e}\right)_{\mathrm{RC}-\mathrm{NLO}} & =\frac{1}{1+3 g_{A}^{2}} \frac{\alpha}{\pi} \frac{E_{e}}{m_{N}}\left(\left(1-g_{A}\right) g_{V}\left(E_{e}\right)+\left(1+2 g_{A}\right) h_{A}\left(E_{e}\right)\right), \\
K_{n}\left(E_{e}\right)_{\mathrm{N}^{2} \mathrm{~L} 0} & =3 \frac{E_{e}}{m_{N}} \bar{K}_{n}\left(E_{e}\right)_{\mathrm{NLO}}-3 \frac{E_{e}}{m_{N}} \bar{A}\left(E_{e}\right)_{\mathrm{NLO}}, \\
K_{n}\left(E_{e}\right)_{\mathrm{WC}} & =-A_{0} \frac{\pi \alpha}{\beta^{3}} \frac{E_{0}-E_{e}}{m_{N}} .
\end{aligned}
$$

For the correlation coefficient $Q_{n}\left(E_{e}\right)$ we obtain the following expression:

$$
\begin{aligned}
Q_{n}\left(E_{e}\right)= & \frac{1}{1+3 g_{A}^{2}} \frac{E_{0}}{m_{N}}\left[\left(g_{A}^{2}+(\kappa+2) g_{A}+(\kappa+1)\right)-\left(7 g_{A}^{2}+(\kappa+8) g_{A}+(\kappa+1)\right) \frac{E_{e}}{E_{0}}\right] \\
& +Q_{n}\left(E_{e}\right)_{\mathrm{RC}-\mathrm{NLO}}+Q_{n}\left(E_{e}\right)_{\mathrm{RC}-\mathrm{PhV}}+Q_{n}\left(E_{e}\right)_{\mathrm{N}^{2} \mathrm{LO}}+Q_{n}\left(E_{e}\right)_{\mathrm{WC}},
\end{aligned}
$$

where the corrections $Q_{n}\left(E_{e}\right)_{\mathrm{RC}-\mathrm{NLO}}, Q_{n}\left(E_{e}\right)_{\mathrm{RC}-\mathrm{PhV}}, Q_{n}\left(E_{e}\right)_{\mathrm{N}^{2} \mathrm{~L} 0}$, and $Q_{n}\left(E_{e}\right)_{\mathrm{WC}}$, defined by the $O\left(\alpha E_{e} / m_{N}\right)$ inner and outer radiative corrections, the $O\left(E_{e}^{2} / m_{N}^{2}\right)$ corrections, caused by weak magnetism and proton recoil, and Wilkinson's corrections, respectively, are equal to 


$$
\begin{aligned}
Q_{n}\left(E_{e}\right)_{\mathrm{RC}-\mathrm{NLO}} & =0, \\
Q_{n}\left(E_{e}\right)_{\mathrm{RC}-\mathrm{PhV}} & =-3 B_{0} \frac{\alpha}{\pi} \frac{E_{e}}{m_{N}} \bar{g}_{n}\left(E_{e}\right), \\
Q_{n}\left(E_{e}\right)_{\mathrm{N} 2 \mathrm{~L} 0} & =-12 \frac{E_{e}^{2}}{m_{N}^{2}} B_{0}\left(1-\frac{1}{8} \frac{E_{0}}{E_{e}}\right)+3 \frac{E_{e}}{m_{N}} \bar{Q}_{n}\left(E_{e}\right)_{\mathrm{NLO}}-3 \frac{E_{e}}{m_{N}} \bar{B}\left(E_{e}\right)_{\mathrm{NLO}}-Q_{n}\left(E_{e}\right)_{\mathrm{NLO}} \zeta\left(E_{e}\right)_{\mathrm{NLO}}, \\
Q_{n}\left(E_{e}\right)_{\mathrm{WC}} & =-B_{0} \frac{\pi \alpha}{\beta^{3}} \frac{E_{0}-E_{e}}{m_{N}} .
\end{aligned}
$$

Now we are able to give the analytical expression for the correlation coefficient $A^{(\beta)}\left(E_{e}\right)$ defined by [15]

$$
A^{(\beta)}\left(E_{e}\right)=A\left(E_{e}\right)+\frac{1}{3} Q_{n}\left(E_{e}\right) .
$$

This correlation coefficient is responsible for the electron (beta) asymmetry in the neutron beta decay [5] (see also [11]). The correlation coefficient $A^{(\beta)}\left(E_{e}\right)$ we give in the following form [15] (see also [11]):

$$
\begin{aligned}
A^{(\beta)}\left(E_{e}\right)= & A_{0}\left\{1+\frac{\alpha}{\pi} f_{n}\left(E_{e}\right)+\frac{g_{A}+\kappa+1}{g_{A}\left(1-g_{A}\right)\left(1+3 g_{A}^{2}\right)} \frac{E_{0}}{m_{N}}\left(A_{1}^{(\beta)}+A_{2}^{(\beta)} \frac{E_{e}}{E_{0}}+A_{3}^{(\beta)} \frac{E_{0}}{E_{e}}\right)\right\} \\
& +A^{(\beta)}\left(E_{e}\right)_{\mathrm{RC}-\mathrm{NLO}}+A^{(\beta)}\left(E_{e}\right)_{\mathrm{RC}-\mathrm{PhV}}+A^{(\beta)}\left(E_{e}\right)_{\mathrm{N}^{2} \mathrm{LO}}+A^{(\beta)}\left(E_{e}\right)_{\mathrm{WC}} .
\end{aligned}
$$

The function $f_{n}\left(E_{e}\right)$ is given in Eq. (A19). The coefficients $A_{1}^{(\beta)}, A_{2}^{(\beta)}$, and $A_{3}^{(\beta)}$ are equal to [15] (see also [11])

$$
\begin{aligned}
& A_{1}^{(\beta)}=g_{A}^{2}+\frac{2}{3} g_{A}-\frac{1}{3}, \\
& A_{2}^{(\beta)}=-g_{A}^{3}-3 g_{A}^{2}-\frac{5}{3} g_{A}+\frac{1}{3}, \\
& A_{3}^{(\beta)}=2 g_{A}^{2}\left(1-g_{A}\right) \frac{m_{e}^{2}}{E_{0}^{2}},
\end{aligned}
$$

and the terms $A^{(\beta)}\left(E_{e}\right)_{\mathrm{RC}-\mathrm{NLO}}, A^{(\beta)}\left(E_{e}\right)_{\mathrm{RC}-\mathrm{PhV}}, A^{(\beta)}\left(E_{e}\right)_{\mathrm{N}^{2} \mathrm{~L} 0}$, and $A^{(\beta)}\left(E_{e}\right)_{\mathrm{WC}}$, defined by the radiative corrections $O\left(\alpha E_{e} / m_{N}\right)$, the corrections $O\left(E_{e}^{2} / m_{N}^{2}\right)$, caused by weak magnetism and proton recoil, and Wilkinson's corrections, respectively, are equal to

$$
\begin{aligned}
A^{(\beta)}\left(E_{e}\right)_{\mathrm{RC}-\mathrm{NLO}}= & \frac{1}{1+3 g_{A}^{2}} \frac{\alpha}{\pi} \frac{E_{e}}{m_{N}}\left(g_{A} f_{V}\left(E_{e}\right)+\sqrt{1-\beta^{2}} g_{S}\left(E_{e}\right)+g_{V}\left(E_{e}\right)-g_{A} h_{A}\left(E_{e}\right)+g_{A}\left(1-2 g_{A}\right) f_{A}\left(E_{e}\right)\right) \\
& +\frac{1}{1+3 g_{A}^{2}} g_{A}\left(1-2 g_{A}\right) \frac{\alpha}{\pi} \frac{5}{2} \frac{m_{N}^{2}}{M_{W}^{2}} \ln \frac{M_{W}^{2}}{m_{N}^{2}}+\frac{1}{1+3 g_{A}^{2}} \frac{\alpha}{\pi}\left(g_{A} \bar{g}_{\mathrm{st}}\left(E_{e}\right)+\left(1-2 g_{A}\right) \bar{f}_{\mathrm{st}}\left(E_{e}\right)\right)-A_{0} \zeta\left(E_{e}\right)_{\mathrm{RC}-\mathrm{NLO}}, \\
A^{(\beta)}\left(E_{e}\right)_{\mathrm{RC}-\mathrm{PhV}}= & \frac{1}{3} Q_{n}\left(E_{e}\right)_{\mathrm{RC}-\mathrm{PhV}}=-B_{0} \frac{\alpha}{\pi} \frac{E_{e}}{m_{N}} \bar{g}_{n}\left(E_{e}\right), \\
A^{(\beta)}\left(E_{e}\right)_{\mathrm{N}^{2} \mathrm{LO}}= & A\left(E_{e}\right)_{\mathrm{N}^{2} \mathrm{LO}}+\frac{1}{3} Q_{n}\left(E_{e}\right)_{\mathrm{N}^{2} \mathrm{LO}}, \\
A^{(\beta)}\left(E_{e}\right)_{\mathrm{WC}}= & 3.76 \times 10^{-4} A_{0}-\frac{1}{3} B_{0} \frac{\pi \alpha}{\beta^{3}} \frac{E_{0}-E_{e}}{m_{N}} .
\end{aligned}
$$

The functions $\zeta\left(E_{e}\right)_{\mathrm{NLO}}, a\left(E_{e}\right)_{\mathrm{NLO}}, A\left(E_{e}\right)_{\mathrm{NLO}}, B\left(E_{e}\right)_{\mathrm{NLO}}$, and $Q_{n}\left(E_{e}\right)_{\mathrm{NLO}}$ are given in Eq. (B2).

For the experimental analysis of the antineutrino asymmetry in the neutron beta decay one has to use Eqs. (27) and (28) in Ref. [11] and the correlation coefficients $a\left(E_{e}\right), A\left(E_{e}\right), B\left(E_{e}\right), K_{n}\left(E_{e}\right)$, and $Q_{n}\left(E_{e}\right)$ given in this Appendix. For the account for the contribution of the Fierz interference term $b$ in the antineutrino asymmetry one may use Eqs. (19) and (20) in Ref. [40], where the correlation coefficients $X\left(E_{e}\right)$ are replaced by $X\left(E_{e}\right) /\left(1+b m_{e} / E_{e}\right)$ for $X\left(E_{e}\right)=a\left(E_{e}\right), A\left(E_{e}\right), B\left(E_{e}\right), K_{n}\left(E_{e}\right)$, and $Q_{n}\left(E_{e}\right)$, respectively. 
For the correlation coefficients $G\left(E_{e}\right), H\left(E_{e}\right), N\left(E_{e}\right), Q_{e}\left(E_{e}\right), K_{e}\left(E_{e}\right), S\left(E_{e}\right), T\left(E_{e}\right)$, and $U\left(E_{e}\right)$ as functions of the electron energy $E_{e}$ and the axial coupling constant $g_{A}$ we give the analytical expressions in the following form:

$$
\begin{aligned}
G\left(E_{e}\right) & =-\left(1+\frac{\alpha}{\pi} f_{n}\left(E_{e}\right)\right)+G\left(E_{e}\right)_{\mathrm{NLO}}+G\left(E_{e}\right)_{\mathrm{RC}-\mathrm{NLO}}+G\left(E_{e}\right)_{\mathrm{N}^{2} \mathrm{LO}}+G\left(E_{e}\right)_{\mathrm{WC}}, \\
H\left(E_{e}\right) & =-\frac{m_{e}}{E_{e}} a_{0}\left(1+\frac{\alpha}{\pi} h_{n}^{(1)}\left(E_{e}\right)\right)+H\left(E_{e}\right)_{\mathrm{NLO}}+H\left(E_{e}\right)_{\mathrm{RC}-\mathrm{NLO}}+H\left(E_{e}\right)_{\mathrm{N} 2 \mathrm{LO}}+H\left(E_{e}\right)_{\mathrm{WC}}, \\
N\left(E_{e}\right) & =-\frac{m_{e}}{E_{e}} A_{0}\left(1+\frac{\alpha}{\pi} h_{n}^{(1)}\left(E_{e}\right)\right)+N\left(E_{e}\right)_{\mathrm{NLO}}+N\left(E_{e}\right)_{\mathrm{RC}-\mathrm{NLO}}+N\left(E_{e}\right)_{\mathrm{N} 2 \mathrm{LO}}+N\left(E_{e}\right)_{\mathrm{WC}}, \\
Q_{e}\left(E_{e}\right) & =-A_{0}\left(1+\frac{\alpha}{\pi} h_{n}^{(2)}\left(E_{e}\right)\right)+Q_{e}\left(E_{e}\right)_{\mathrm{NLO}}+Q_{e}\left(E_{e}\right)_{\mathrm{RC}-\mathrm{NLO}}+Q_{e}\left(E_{e}\right)_{\mathrm{RC}-\mathrm{PhV}}+Q_{e}\left(E_{e}\right)_{\mathrm{N}^{2} \mathrm{LO}}+Q_{e}\left(E_{e}\right)_{\mathrm{WC}}, \\
K_{e}\left(E_{e}\right) & =-a_{0}\left(1+\frac{\alpha}{\pi} h_{n}^{(2)}\left(E_{e}\right)\right)+K_{e}\left(E_{e}\right)_{\mathrm{NLO}}+K_{e}\left(E_{e}\right)_{\mathrm{RC}-\mathrm{NLO}}+K_{e}\left(E_{e}\right)_{\mathrm{RC}-\mathrm{PhV}}+K_{e}\left(E_{e}\right)_{\mathrm{N}^{2} \mathrm{LO}}+K_{e}\left(E_{e}\right)_{\mathrm{WC}}, \\
S\left(E_{e}\right) & =S\left(E_{e}\right)_{\mathrm{NLO}}+S\left(E_{e}\right)_{\mathrm{RC}-\mathrm{NLO}}+S\left(E_{e}\right)_{\mathrm{N}^{2} \mathrm{LO}}, \\
T\left(E_{e}\right) & =-B_{0}\left(1+\frac{\alpha}{\pi} f_{n}\left(E_{e}\right)\right)+T\left(E_{e}\right)_{\mathrm{NLO}}+T\left(E_{e}\right)_{\mathrm{RC}-\mathrm{NLO}}+T\left(E_{e}\right)_{\mathrm{N}^{2} \mathrm{LO}}+T\left(E_{e}\right)_{\mathrm{WC}}, \\
U\left(E_{e}\right) & =U\left(E_{e}\right)_{\mathrm{RC}-\mathrm{NLO}}+U\left(E_{e}\right)_{\mathrm{N}^{2} \mathrm{LO}} .
\end{aligned}
$$

The analytical expressions of the functions $f_{n}\left(E_{e}\right), h_{n}^{(1)}\left(E_{e}\right)$, and $h_{n}^{(2)}\left(E_{e}\right)$ are given in Eq. (A19). The corrections $X\left(E_{e}\right)_{\mathrm{NLO}}, X\left(E_{e}\right)_{\mathrm{RC}-\mathrm{NLO}}, X\left(E_{e}\right)_{\mathrm{RC}-\mathrm{PhV}}, X\left(E_{e}\right)_{\mathrm{N}^{2} \mathrm{LO}}$, and $X\left(E_{e}\right)_{\mathrm{WC}}$ for $X=G, H, N, Q_{e}, K_{e}, S, T, U$ are adduced in Eq. (B2), Eq. (A20), Eq. (D10), Eq. (B4), and Eq. (C8), respectively.

For the practical applications and numerical analysis the analytical expressions of the correlation function $\zeta\left(E_{e}\right)$ and correlation coefficients $X\left(E_{e}\right)$ for $X=a, A, B ; \ldots, U$ and $A^{(\beta)}\left(E_{e}\right)$ are programmed in [46].

\section{APPENDIX F: CONTRIBUTIONS TO THE ELECTRON-ENERGY AND ANGULAR DISTRIBUTION OF THE NEUTRON BETA DECAY WITH CORRELATION STRUCTURES BEYOND EQ. (1)}

The electron-energy and angular distribution of the neutron beta decay Eq. (1), supplemented by the contributions with correlation structures beyond the standard ones, takes the form

$$
\begin{aligned}
\frac{d^{5} \lambda_{n}\left(E_{e}, \vec{k}_{e}, \vec{k}_{\bar{\nu}}, \vec{\xi}_{n}, \vec{\xi}_{e}\right)}{d E_{e} d \Omega_{e} d \Omega_{\bar{\nu}}} & \left(1+3 g_{A}^{2}\right) \frac{\left|G_{V}\right|^{2}}{16 \pi^{5}}\left(E_{0}-E_{e}\right)^{2} \sqrt{E_{e}^{2}-m_{e}^{2}} E_{e} F\left(E_{e}, Z=1\right) \zeta\left(E_{e}\right)\left\{1+b\left(E_{e}\right) \frac{m_{e}}{E_{e}}\right. \\
= & +a\left(E_{e}\right) \frac{\vec{k}_{e} \cdot \vec{k}_{\bar{\nu}}}{E_{e} E_{\bar{\nu}}}+A\left(E_{e}\right) \frac{\vec{\xi}_{n} \cdot \vec{k}_{e}}{E_{e}}+B\left(E_{e}\right) \frac{\vec{\xi}_{n} \cdot \vec{k}_{\bar{\nu}}}{E_{\bar{\nu}}}+K_{n}\left(E_{e}\right) \frac{\left(\vec{\xi}_{n} \cdot \vec{k}_{e}\right)\left(\vec{k}_{e} \cdot \vec{k}_{\bar{\nu}}\right)}{E_{e}^{2} E_{\bar{\nu}}}+Q_{n}\left(E_{e}\right) \frac{\left(\vec{\xi}_{n} \cdot \vec{k}_{\bar{\nu}}\right)\left(\vec{k}_{e} \cdot \vec{k}_{\bar{\nu}}\right)}{E_{e} E_{\bar{\nu}}^{2}} \\
& +D\left(E_{e}\right) \frac{\vec{\xi}_{n} \cdot\left(\vec{k}_{e} \times \vec{k}_{\bar{\nu}}\right)}{E_{e} E_{\bar{\nu}}}+G\left(E_{e}\right) \frac{\vec{\xi}_{e} \cdot \vec{k}_{e}}{E_{e}}+H\left(E_{e}\right) \frac{\vec{\xi}_{e} \cdot \vec{k}_{\bar{\nu}}}{E_{\bar{\nu}}}+N\left(E_{e}\right) \vec{\xi}_{n} \cdot \vec{\xi}_{e}+Q_{e}\left(E_{e}\right) \frac{\left(\vec{\xi}_{n} \cdot \vec{k}_{e}\right)\left(\vec{k}_{e} \cdot \vec{\xi}_{e}\right)}{\left(E_{e}+m_{e}\right) E_{e}} \\
& +K_{e}\left(E_{e}\right) \frac{\left(\vec{\xi}_{e} \cdot \vec{k}_{e}\right)\left(\vec{k}_{e} \cdot \vec{k}_{\bar{\nu}}\right)}{\left(E_{e}+m_{e}\right) E_{e} E_{\bar{\nu}}}+R\left(E_{e}\right) \frac{\vec{\xi}_{n} \cdot\left(\vec{k}_{e} \times \vec{\xi}_{e}\right)}{E_{e}}+L\left(E_{e}\right) \frac{\vec{\xi}_{e} \cdot\left(\vec{k}_{e} \times \vec{k}_{\bar{\nu}}\right)}{E_{e} E_{\bar{\nu}}}+S\left(E_{e}\right) \frac{\left(\vec{\xi}_{n} \cdot \vec{\xi}_{e}\right)\left(\vec{k}_{e} \cdot \vec{k}_{\bar{\nu}}\right)}{E_{e} E_{\bar{\nu}}} \\
& \left.+T\left(E_{e}\right) \frac{\left(\vec{\xi}_{n} \cdot \vec{k}_{\bar{\nu}}\right)\left(\vec{\xi}_{e} \cdot \vec{k}_{e}\right)}{E_{e} E_{\bar{\nu}}}+U\left(E_{e}\right) \frac{\left(\vec{\xi}_{n} \cdot \vec{k}_{e}\right)\left(\vec{\xi}_{e} \cdot \vec{k}_{\bar{\nu}}\right)}{E_{e} E_{\bar{\nu}}}+V\left(E_{e}\right) \frac{\vec{\xi}_{n} \cdot\left(\vec{\xi}_{e} \times \vec{k}_{\bar{\nu}}\right)}{E_{\bar{\nu}}}+W\left(E_{e}\right) \frac{\vec{\xi}_{n} \cdot\left(\vec{k}_{e} \times \vec{k}_{\bar{\nu}}\right)\left(\vec{\xi}_{e} \cdot \vec{k}_{e}\right)}{\left(E_{e}+m_{e}\right) E_{e} E_{\bar{\nu}}}\right\} \\
& +\left.\frac{d^{5} \lambda_{n}\left(E_{e}, \vec{k}_{e}, \vec{k}_{\bar{\nu}}, \vec{\xi}_{n}, \vec{\xi}_{e}\right)}{d E_{e} d \Omega_{e} d \Omega_{\bar{\nu}}}\right|_{\mathrm{RC}-\mathrm{NLO}}+\left.\frac{d^{5} \lambda_{n}\left(E_{e}, \vec{k}_{e}, \vec{k}_{\bar{\nu}}, \vec{\xi}_{n}, \vec{\xi}_{e}\right)}{d E_{e} d \Omega_{e} d \Omega_{\bar{\nu}}}\right|_{\mathrm{NLO}}+\left.\sum_{m=1}^{4} \frac{d^{5} \lambda_{n}^{(m)}\left(E_{e}, \vec{k}_{e}, \vec{k}_{\bar{\nu}}, \vec{\xi}_{n}, \vec{\xi}_{e}\right)}{d E_{e} d \Omega_{e} d \Omega_{\bar{\nu}}}\right|_{N^{2} \mathrm{LO}} \\
& \left.+\left.\frac{d^{5} \lambda_{n}\left(E_{e}, \vec{k}_{e}, \vec{k}_{\bar{\nu}}, \vec{\xi}_{n}, \vec{\xi}_{e}\right)}{d E_{e} d \Omega_{e} d \Omega_{\bar{\nu}}}\right|_{\mathrm{W}}\right\}, \quad
\end{aligned}
$$


where the last seven terms are defined by the following expressions (see also [24]):

$$
\begin{aligned}
\left.\frac{d^{5} \lambda_{n}\left(E_{e}, \vec{k}_{e}, \vec{k}_{\bar{\nu}}, \vec{\xi}_{n}, \vec{\xi}_{e}\right)}{d E_{e} d \Omega_{e} d \Omega_{\bar{\nu}}}\right|_{\mathrm{RC}-\mathrm{NLO}}= & \left(1+3 g_{A}^{2}\right) \frac{\left|G_{V}\right|^{2}}{16 \pi^{5}}\left(E_{0}-E_{e}\right)^{2} \sqrt{E_{e}^{2}-m_{e}^{2}} E_{e} F\left(E_{e}, Z=1\right) \zeta\left(E_{e}\right) \\
& \times\left\{\frac{2 E_{e}}{1+3 g_{A}^{2}}\left(2 g_{A} U_{5}-\left(1-g_{A}\right) U_{7}-\left(1+g_{A}\right) U_{8}\right) \frac{\left(\vec{\xi}_{n} \cdot \vec{k}_{e}\right)\left(\vec{\xi}_{e} \cdot \vec{k}_{e}\right)\left(\vec{k}_{e} \cdot \vec{k}_{\bar{\nu}}\right)}{\left(E_{e}+m_{e}\right) E_{e}^{2} E_{\bar{\nu}}}-\frac{2 E_{e}}{1+3 g_{A}^{2}}\left(1+g_{A}\right) U_{6}\right. \\
& \times\left[\left(\frac{\left(\vec{\xi}_{n} \cdot \vec{k}_{\bar{\nu}}\right)\left(\vec{\xi}_{e} \cdot \vec{k}_{\bar{\nu}}\right)}{E_{\bar{\nu}}^{2}}-\frac{1}{3} \vec{\xi}_{e} \cdot \vec{\xi}_{e}\right)+\left(-\frac{\left(\vec{\xi}_{n} \cdot \vec{k}_{\bar{\nu}}\right)\left(\vec{\xi}_{e} \cdot \vec{k}_{e}\right)\left(\vec{k}_{e} \cdot \vec{k}_{\bar{\nu}}\right)}{\left(E_{e}+m_{e}\right) E_{e} E_{\bar{\nu}}^{2}}+\frac{1}{3} \frac{\left(\vec{\xi}_{n} \cdot \vec{k}_{e}\right)\left(\vec{\xi}_{e} \cdot \vec{k}_{e}\right)}{\left(E_{e}+m_{e}\right) E_{e}}\right)\right] \\
& \left.+3 B_{0} \frac{\alpha E_{e}}{\pi m_{N}} \bar{g}_{n}\left(E_{e}\right)\left(\frac{\left(\vec{\xi}_{n} \cdot \vec{k}_{\bar{\nu}}\right)\left(\vec{\xi}_{e} \cdot \vec{k}_{e}\right)\left(\vec{k}_{e} \cdot \vec{k}_{\bar{\nu}}\right)}{E_{e}^{2} E_{\bar{\nu}}^{2}}-\frac{1}{3} \frac{\left(\vec{\xi}_{n} \cdot \vec{k}_{e}\right)\left(\vec{\xi}_{e} \cdot \vec{k}_{e}\right)}{E_{e}^{2}}\right)\right\},
\end{aligned}
$$

where the functions $U_{5}, U_{6}, U_{7}$, and $U_{8}$ are given in Eq. (A4), and

$$
\begin{aligned}
\left.\frac{d^{5} \lambda_{n}\left(E_{e}, \vec{k}_{e}, \vec{k}_{\bar{\nu}}, \vec{\xi}_{n}, \vec{\xi}_{e}\right)}{d E_{e} d \Omega_{e} d \Omega_{\bar{\nu}}}\right|_{\mathrm{NLO}}= & \left(1+3 g_{A}^{2}\right) \frac{\left|G_{V}\right|^{2}}{16 \pi^{5}}\left(E_{0}-E_{e}\right)^{2} \sqrt{E_{e}^{2}-m_{e}^{2}} E_{e} F\left(E_{e}, Z=1\right) \zeta\left(E_{e}\right) \\
& \times \frac{E_{e}}{m_{N}}\left\{-3 \frac{1-g_{A}^{2}}{1+3 g_{A}^{2}}\left(\frac{\left(\vec{k}_{e} \cdot \vec{k}_{\bar{\nu}}\right)^{2}}{E_{e}^{2} E_{\bar{\nu}}^{2}}-\frac{1}{3} \frac{k_{e}^{2}}{E_{e}^{2}}\right)+3 \frac{1-g_{A}^{2}}{1+3 g_{A}^{2}}\left(\frac{\left(\vec{\xi}_{e} \cdot \vec{k}_{\bar{\nu}}\right)\left(\vec{k}_{e} \cdot \vec{k}_{\nu}\right)}{E_{e} E_{\bar{\nu}}^{2}}-\frac{1}{3} \frac{\vec{\xi}_{e} \cdot \vec{k}_{e}}{E_{e}}\right) \frac{m_{e}}{E_{e}}\right. \\
& \left.+3 \frac{1-g_{A}^{2}}{1+3 g_{A}^{2}}\left(\frac{\left(\vec{\xi}_{e} \cdot \vec{k}_{e}\right)\left(\vec{k}_{e} \cdot \vec{k}_{\bar{\nu}}\right)^{2}}{\left(E_{e}+m_{e}\right) E_{e}^{2} E_{\bar{\nu}}^{2}}-\frac{1}{3}\left(1-\frac{m_{e}}{E_{e}}\right) \frac{\vec{\xi}_{e} \cdot \vec{k}_{e}}{E_{e}}\right)\right\}
\end{aligned}
$$

and

$$
\begin{aligned}
\left.\frac{d^{5} \lambda_{n}^{(1)}\left(E_{e}, \vec{k}_{e}, \vec{k}_{\bar{\nu}}, \vec{\xi}_{n}, \vec{\xi}_{e}\right)}{d E_{e} d \Omega_{e} d \Omega_{\bar{\nu}}}\right|_{N^{2} \mathrm{LO}} \\
=\left(1+3 g_{A}^{2}\right) \frac{\left|G_{V}\right|^{2}}{16 \pi^{5}}\left(E_{0}-E_{e}\right)^{2} \sqrt{E_{e}^{2}-m_{e}^{2}} E_{e} F\left(E_{e}, Z=1\right) \zeta\left(E_{e}\right) \\
\quad \times\left\{\frac { E _ { 0 } ^ { 2 } } { 2 m _ { N } ^ { 2 } } \left\{-\frac{1}{1+3 g_{A}^{2}}\left(g_{A}+2 \kappa+1\right) \frac{E_{e} E_{\bar{\nu}}}{E_{0}^{2}}\left(\frac{\left(\vec{k}_{e} \cdot \vec{k}_{\bar{\nu}}\right)^{2}}{E_{e}^{2} E_{\bar{\nu}}^{2}}-\frac{1}{3} \frac{k_{e}^{2}}{E_{e}^{2}}\right)-\frac{1}{1+3 g_{A}^{2}}\left(g_{A}^{2}+2 \kappa+1\right) \frac{E_{\bar{\nu}}}{E_{e}+m_{e}}\right.\right. \\
\quad \times\left(\frac{\left(\vec{k}_{e} \cdot \vec{k}_{\bar{\nu}}\right)^{2}}{E_{e}^{2} E_{\bar{\nu}}^{2}}-\frac{1}{3} \frac{k_{e}^{2}}{E_{e}^{2}}\right) \frac{\vec{\xi}_{e} \cdot \vec{k}_{e}}{E_{e}}-\frac{1}{1+3 g_{A}^{2}}\left(g_{A}^{2}+2 \kappa+1\right) \frac{m_{e} E_{\bar{\nu}}}{E_{0}^{2}}\left(\frac{\left(\vec{\xi}_{e} \cdot \vec{k}_{\bar{\nu}}\right)\left(\vec{k}_{e} \cdot \vec{k}_{\bar{\nu}}\right)}{E_{e} E_{\bar{\nu}}^{2}}-\frac{1}{3} \frac{\vec{\xi}_{e} \cdot \vec{k}_{e}}{E_{e}}\right) \\
\quad+\frac{1}{1+3 g_{A}^{2}} 2 \kappa g_{A} \frac{E_{e} E_{\bar{\nu}}}{E_{0}^{2}}\left(\frac{\left(\vec{\xi}_{n} \cdot \vec{k}_{\bar{\nu}}\right)\left(\vec{k}_{e} \cdot \vec{\xi}_{e}\right)\left(\vec{k}_{e} \cdot \vec{k}_{\bar{\nu}}\right)}{E_{e}^{2} E_{\bar{\nu}}^{2}}-\frac{1}{3} \frac{\left(\vec{\xi}_{n} \cdot \vec{k}_{e}\right)\left(\vec{k}_{e} \cdot \vec{\xi}_{e}\right)}{E_{e}^{2}}\right)+\frac{1}{1+3 g_{A}^{2}}\left(2 \kappa g_{A} \frac{E_{e} E_{\bar{\nu}}}{E_{0}^{2}}\right) \\
\quad \times \frac{\left(\vec{\xi}_{n} \cdot \vec{k}_{e}\right)\left(\vec{k}_{e} \cdot \vec{\xi}_{e}\right)\left(\vec{k}_{e} \cdot \vec{k}_{\bar{\nu}}\right)}{\left(E_{e}+m_{e}\right) E_{e}^{2} E_{\bar{\nu}}}+\left\{\frac{8 g_{A}}{1+3 g_{A}^{2}}\left(\frac{E_{0}^{2}}{M_{V}^{2}}+\left(1+2 g_{A}\right) \frac{E_{0}^{2}}{M_{A}^{2}}\right)\left(\frac{\left(\vec{\xi}_{n} \cdot \vec{k}_{\bar{\nu}}\right)\left(\vec{k}_{e} \cdot \vec{k}_{\bar{\nu}}\right)}{E_{e} E_{\bar{\nu}}^{2}}-\frac{1}{3} \frac{\vec{\xi}_{n} \cdot \vec{k}_{e}}{E_{e}}\right)\right. \\
\left.\left.\quad-\frac{8 g_{A}}{1+3 g_{A}^{2}}\left(\frac{E_{0}^{2}}{M_{V}^{2}}+\left(1+2 g_{A}\right) \frac{E_{0}^{2}}{M_{A}^{2}}\right)\left(\frac{\left(\vec{\xi}_{n} \cdot \vec{k}_{\bar{\nu}}\right)\left(\vec{\xi}_{e} \cdot \vec{k}_{e}\right)\left(\vec{k}_{e} \cdot \vec{k}_{\bar{\nu}}\right)}{E_{e}^{2} E_{\bar{\nu}}^{2}}-\frac{1}{3} \frac{\left(\vec{\xi}_{n} \cdot \vec{k}_{e}\right)\left(\vec{\xi}_{e} \cdot \vec{k}_{e}\right)}{E_{e}^{2}}\right)\right\} \frac{E_{e}}{E_{0}}\left(1-\frac{E_{e}}{E_{0}}\right)\right\}
\end{aligned}
$$

and 


$$
\begin{aligned}
& \left.\frac{d^{5} \lambda_{n}^{(2)}\left(E_{e}, \vec{k}_{e}, \vec{k}_{\bar{\nu}}, \vec{\xi}_{n}, \vec{\xi}_{e}\right)}{d E_{e} d \Omega_{e} d \Omega_{\bar{\nu}}}\right|_{\mathrm{N}^{2} \mathrm{LO}} \\
& =\left(1+3 g_{A}^{2}\right) \frac{\left|G_{V}\right|^{2}}{16 \pi^{5}}\left(E_{0}-E_{e}\right)^{2} \sqrt{E_{e}^{2}-m_{e}^{2}} E_{e} F\left(E_{e}, Z=1\right) \zeta\left(E_{e}\right) \\
& \times 6 \frac{E_{e}^{2}}{m_{N}^{2}}\left\{\left[1-2 \frac{1-g_{A}^{2}}{1+3 g_{A}^{2}}\left(1-\frac{1}{8} \frac{E_{0}}{E_{e}}\right)\right]\left(\frac{\left(\vec{k}_{e} \cdot \vec{k}_{\bar{\nu}}\right)^{2}}{E_{e}^{2} E_{\bar{\nu}}^{2}}-\frac{1}{3} \frac{k_{e}^{2}}{E_{e}^{2}}\right)+2 \frac{g_{A}\left(1-g_{A}\right)}{1+3 g_{A}^{2}}\left(\frac{\left(\vec{k}_{e} \cdot \vec{k}_{\bar{\nu}}\right)^{2}}{E_{e}^{2} E_{\bar{\nu}}^{2}}-\frac{1}{3} \frac{k_{e}^{2}}{E_{e}^{2}}\right) \frac{\vec{\xi}_{n} \cdot \vec{k}_{e}}{E_{e}}\right. \\
& +\left[-1+2 \frac{1-g_{A}^{2}}{1+3 g_{A}^{2}}\left(1-\frac{1}{8} \frac{E_{0}}{E_{e}}\right) \frac{E_{e}}{E_{e}+m_{e}}\right]\left(\frac{\left(\vec{k}_{e} \cdot \vec{k}_{\bar{\nu}}\right)^{2}}{E_{e}^{2} E_{\bar{\nu}}^{2}}-\frac{1}{3} \frac{k_{e}^{2}}{E_{e}^{2}}\right) \frac{\vec{\xi}_{e} \cdot \vec{k}_{e}}{E_{e}}+2 \frac{1-g_{A}^{2}}{1+3 g_{A}^{2}} \frac{m_{e}}{E_{e}}\left(1-\frac{1}{8} \frac{E_{0}}{E_{e}}\right) \\
& \times\left(\frac{\left(\vec{\xi}_{e} \cdot \vec{k}_{\bar{\nu}}\right)\left(\vec{k}_{e} \cdot \vec{k}_{\bar{\nu}}\right)}{E_{e} E_{\bar{\nu}}^{2}}-\frac{1}{3} \frac{\vec{\xi}_{e} \cdot \vec{k}_{e}}{E_{e}}\right)-2 \frac{g_{A}\left(1-g_{A}\right)}{1+3 g_{A}^{2}} \frac{m_{e}}{E_{e}}\left(\frac{\left(\vec{k}_{e} \cdot \vec{k}_{\bar{\nu}}\right)^{2}}{E_{e}^{2} E_{\bar{\nu}}^{2}}-\frac{1}{3} \frac{k_{e}^{2}}{E_{e}^{2}}\right)\left(\vec{\xi}_{n} \cdot \vec{\xi}_{e}\right)+4 \frac{g_{A}\left(1+g_{A}\right)}{1+3 g_{A}^{2}}\left(1-\frac{1}{8} \frac{E_{0}}{E_{e}}\right) \\
& \times\left(\frac{\left(\vec{\xi}_{n} \cdot \vec{k}_{\bar{\nu}}\right)\left(\vec{k}_{e} \cdot \vec{\xi}_{e}\right)\left(\vec{k}_{e} \cdot \vec{k}_{\bar{\nu}}\right)}{E_{e}^{2} E_{\bar{\nu}}^{2}}-\frac{1}{3} \frac{\left(\vec{\xi}_{n} \cdot \vec{k}_{e}\right)\left(\vec{k}_{e} \cdot \vec{\xi}_{e}\right)}{E_{e}^{2}}\right)+2 \frac{g_{A}\left(1-g_{A}\right)}{1+3 g_{A}^{2}} \frac{m_{e}}{E_{e}}\left(\frac{\left(\vec{k}_{e} \cdot \vec{k}_{\bar{\nu}}\right)^{2}}{E_{e}^{2} E_{\bar{\nu}}^{2}}-\frac{1}{3} \frac{k_{e}^{2}}{E_{e}^{2}}\right) \frac{\left(\vec{\xi}_{n} \cdot \vec{k}_{e}\right)\left(\vec{k}_{e} \cdot \vec{\xi}_{e}\right)}{\left(E_{e}+m_{e}\right) E_{e}} \\
& +4 \frac{g_{A}\left(1-g_{A}\right)}{1+3 g_{A}^{2}}\left(1-\frac{1}{8} \frac{E_{0}}{E_{e}}\right) \frac{\left(\vec{\xi}_{n} \cdot \vec{k}_{e}\right)\left(\vec{k}_{e} \cdot \vec{\xi}_{e}\right)\left(\vec{k}_{e} \cdot \vec{k}_{\bar{\nu}}\right)}{\left(E_{e}+m_{e}\right) E_{e}^{2} E_{\bar{\nu}}}+2 \frac{g_{A}\left(1+g_{A}\right)}{1+3 g_{A}^{2}} \frac{\left(\vec{\xi}_{n} \cdot \vec{k}_{\bar{\nu}}\right)\left(\vec{k}_{e} \cdot \vec{k}_{\bar{\nu}}\right)^{2}}{E_{e}^{2} E_{\bar{\nu}}^{3}}-2 \frac{g_{A}\left(1+g_{A}\right)}{1+3 g_{A}^{2}} \\
& \left.\times \frac{\left(\vec{\xi}_{n} \cdot \vec{k}_{\bar{\nu}}\right)\left(\vec{k}_{e} \cdot \vec{\xi}_{e}\right)\left(\vec{k}_{e} \cdot \vec{k}_{\bar{\nu}}\right)^{2}}{E_{e}^{3} E_{\bar{\nu}}^{3}}-\frac{1-g_{A}^{2}}{1+3 g_{A}^{2}} \frac{m_{e}}{E_{e}} \frac{\left(\vec{\xi}_{e} \cdot \vec{k}_{\bar{\nu}}\right)\left(\vec{k}_{e} \cdot \vec{k}_{\bar{\nu}}\right)^{2}}{E_{e}^{2} E_{\bar{\nu}}^{3}}-\frac{1-g_{A}^{2}}{1+3 g_{A}^{2}} \frac{\left(\vec{\xi}_{e} \cdot \vec{k}_{e}\right)\left(\vec{k}_{e} \cdot \vec{k}_{\bar{\nu}}\right)^{3}}{\left(E_{e}+m_{e}\right) E_{e}^{3} E_{\bar{\nu}}^{3}}-\frac{1-g_{A}^{2}}{1+3 g_{A}^{2}} \frac{\left(\vec{k}_{e} \cdot \vec{k}_{\bar{\nu}}\right)^{3}}{E_{e}^{3} E_{\bar{\nu}}^{3}}\right\}
\end{aligned}
$$

and

$$
\begin{aligned}
& \left.\frac{d^{5} \lambda_{n}^{(3)}\left(E_{e}, \vec{k}_{e}, \vec{k}_{\bar{\nu}}, \vec{\xi}_{n}, \vec{\xi}_{e}\right)}{d E_{e} d \Omega_{e} d \Omega_{\bar{\nu}}}\right|_{\mathrm{N}^{2} \mathrm{LO}} \\
& =\left(1+3 g_{A}^{2}\right) \frac{\left|G_{V}\right|^{2}}{16 \pi^{5}}\left(E_{0}-E_{e}\right)^{2} \sqrt{E_{e}^{2}-m_{e}^{2}} E_{e} F\left(E_{e}, Z=1\right) \zeta\left(E_{e}\right) \\
& \times \frac{E_{0}^{2}}{2 m_{N}^{2}}\left\{-\frac{1}{1+3 g_{A}^{2}}\left[\left(g_{A}^{2}+(\kappa+1)^{2}\right) \frac{E_{\bar{\nu}}}{E_{0}}\right]\left(\frac{\left(\vec{k}_{e} \cdot \vec{k}_{\bar{\nu}}\right)^{2}}{E_{e}^{2} E_{\bar{\nu}}^{2}}-\frac{1}{3} \frac{k_{e}^{2}}{E_{e}^{2}}\right)+\frac{1}{1+3 g_{A}^{2}}\left[\left(g_{A}^{2}+(\kappa+1)^{2}\right) \frac{m_{e}}{E_{0}} \frac{E_{\bar{\nu}}}{E_{0}}\right]\right. \\
& \times\left(\frac{\left(\vec{\xi}_{e} \cdot \vec{k}_{\bar{\nu}}\right)\left(\vec{k}_{e} \cdot \vec{k}_{\bar{\nu}}\right)}{E_{e} E_{\bar{\nu}}^{2}}-\frac{1}{3} \frac{\vec{\xi}_{e} \cdot \vec{k}_{e}}{E_{e}}\right)+\frac{1}{1+3 g_{A}^{2}}\left[\left(g_{A}^{2}+(\kappa+1)^{2}\right) \frac{E_{e} E_{\bar{\nu}}}{E_{0}^{2}}\right]\left(\frac{\left(\vec{k}_{e} \cdot \vec{k}_{\bar{\nu}}\right)^{2}}{E_{e}^{2} E_{\bar{\nu}}^{2}}-\frac{1}{3} \frac{k_{e}^{2}}{E_{e}^{2}}\right) \frac{\vec{\xi}_{e} \cdot \vec{k}_{e}}{E_{e}+m_{e}} \\
& +\frac{1}{1+3 g_{A}^{2}} \frac{m_{e}}{E_{e}}\left[(\kappa+1)\left(g_{A}+(\kappa+1)\right) \frac{E_{\bar{\nu}}^{2}}{E_{0}^{2}}+\left(g_{A}+(\kappa+1)\right) \frac{E_{e} E_{\bar{\nu}}}{E_{0}^{2}}+g_{A}\left(g_{A}+(\kappa+1)\right) \frac{E_{\bar{\nu}}}{E_{0}}\right] \\
& \times\left(\frac{\left(\vec{\xi}_{n} \cdot \vec{k}_{\bar{\nu}}\right)\left(\vec{k}_{\bar{\nu}} \cdot \vec{\xi}_{e}\right)}{E_{\bar{\nu}}^{2}}-\frac{1}{3} \vec{\xi}_{n} \cdot \vec{\xi}_{e}\right)+\frac{1}{1+3 g_{A}^{2}}\left[g_{A}\left(g_{A}+(\kappa+1)\right) \frac{E_{\bar{\nu}}}{E_{0}}+\left(g_{A}+(\kappa+1)\right) \frac{E_{e} E_{\bar{\nu}}}{E_{0}^{2}}\right. \\
& \left.+(\kappa+1)\left(g_{A}+(\kappa+1)\right) \frac{E_{\bar{\nu}}^{2}}{E_{0}^{2}}-(\kappa+2)\left(g_{A}+(\kappa+1)\right) \frac{E_{e} E_{\bar{\nu}}}{E_{0}^{2}}\left(1+\frac{m_{e}}{E_{e}}\right)\right]\left(\frac{\left(\vec{\xi}_{n} \cdot \vec{k}_{\bar{\nu}}\right)\left(\vec{k}_{e} \cdot \vec{k}_{\bar{\nu}}\right)}{E_{e} E_{\bar{\nu}}^{2}}-\frac{1}{3} \frac{\vec{\xi}_{n} \cdot \vec{k}_{e}}{E_{e}}\right) \\
& \times \frac{\vec{k}_{e} \cdot \vec{\xi}_{e}}{E_{e}+m_{e}}+\frac{1}{1+3 g_{A}^{2}}\left[\kappa\left(g_{A}-(\kappa+1)\right) \frac{m_{e}}{E_{0}} \frac{E_{e}}{E_{0}}-g_{A}\left(g_{A}-(\kappa+1)\right) \frac{E_{e}}{E_{0}}+(\kappa+1)\left(g_{A}-(\kappa+1)\right) \frac{E_{e}^{2}}{E_{0}^{2}}\right. \\
& \left.\left.+(\kappa+1)\left(3 g_{A}+(\kappa+1)\right) \frac{E_{e} E_{\bar{\nu}}}{E_{0}^{2}}\right] \frac{\left(\vec{\xi}_{n} \cdot \vec{k}_{e}\right)\left(\vec{k}_{e} \cdot \vec{\xi}_{e}\right)\left(\vec{k}_{e} \cdot \vec{k}_{\bar{\nu}}\right)}{\left(E_{e}+m_{e}\right) E_{e}^{2} E_{\bar{\nu}}}\right\}
\end{aligned}
$$

and 


$$
\begin{aligned}
\left.\frac{d^{5} \lambda_{n}^{(4)}\left(E_{e}, \vec{k}_{e}, \vec{k}_{\bar{\nu}}, \vec{\xi}_{n}, \vec{\xi}_{e}\right)}{d E_{e} d \Omega_{e} d \Omega_{\bar{\nu}}}\right|_{\mathrm{N}^{2} \mathrm{LO}}= & \left(1+3 g_{A}^{2}\right) \frac{\left|G_{V}\right|^{2}}{16 \pi^{5}}\left(E_{0}-E_{e}\right)^{2} \sqrt{E_{e}^{2}-m_{e}^{2}} E_{e} F\left(E_{e}, Z=1\right) \zeta\left(E_{e}\right) \\
& \times 3 \frac{E_{e}}{m_{N}}\left\{-\bar{K}_{n}\left(E_{e}\right)_{\mathrm{NLO}}\left(\frac{\left(\vec{k}_{e} \cdot \vec{k}_{\bar{\nu}}\right)^{2}}{E_{e}^{2} E_{\bar{\nu}}^{2}}-\frac{1}{3} \frac{k_{e}^{2}}{E_{e}^{2}}\right)-\bar{Q}_{n}\left(E_{e}\right)_{\mathrm{NLO}} \frac{\left(\vec{\xi}_{n} \cdot \vec{k}_{\bar{\nu}}\right)\left(\vec{k}_{e} \cdot \vec{k}_{\bar{\nu}}\right)^{2}}{E_{e}^{2} E_{\bar{\nu}}^{3}}-\bar{H}\left(E_{e}\right)_{\mathrm{NLO}}\right. \\
& \times\left(\frac{\left(\vec{\xi}_{e} \cdot \vec{k}_{\bar{\nu}}\right)\left(\vec{k}_{e} \cdot \vec{k}_{\bar{\nu}}\right)}{E_{e} E_{\bar{\nu}}^{2}}-\frac{1}{3} \frac{\vec{\xi}_{e} \cdot \vec{k}_{e}}{E_{e}}\right)-\bar{Q}_{e}\left(E_{e}\right)_{\mathrm{NLO}} \frac{\left(\vec{\xi}_{n} \cdot \vec{k}_{e}\right)\left(\vec{k}_{e} \cdot \vec{\xi}_{e}\right)\left(\vec{k}_{e} \cdot \vec{k}_{\bar{\nu}}\right)}{\left(E_{e}+m_{e}\right) E_{e}^{2} E_{\bar{\nu}}} \\
& \left.-\bar{K}_{e}\left(E_{e}\right)_{\mathrm{NLO}}\left(\frac{\left(\vec{k}_{e} \cdot \vec{k}_{\bar{\nu}}\right)^{2}}{E_{e}^{2} E_{\bar{\nu}}^{2}}-\frac{1}{3} \frac{k_{e}^{2}}{E_{e}^{2}}\right) \frac{\vec{\xi}_{e} \cdot \vec{k}_{e}}{E_{e}+m_{e}}\right\}+\left.3 \frac{E_{e}}{m_{N}}\left(1-\frac{\vec{k}_{e} \cdot \vec{k}_{\bar{\nu}}}{E_{e} E_{\bar{\nu}}}\right) \frac{d^{5} \lambda_{n}\left(E_{e}, \vec{k}_{e}, \vec{k}_{\bar{\nu}}, \vec{\xi}_{n}, \vec{\xi}_{e}\right)}{d E_{e} d \Omega_{e} d \Omega_{\bar{\nu}}}\right|_{\mathrm{NLO}},
\end{aligned}
$$

where $\bar{K}_{n}\left(E_{e}\right)_{\mathrm{NLO}}$ and $\bar{Q}_{n}\left(E_{e}\right)_{\mathrm{NLO}}$ coincide with $K_{n}\left(E_{e}\right)_{\mathrm{NLO}}$ and $Q_{n}\left(E_{e}\right)_{\mathrm{NLO}}$, which are given in Eq. (B2), whereas $\bar{H}\left(E_{e}\right)$, $\bar{Q}_{e}\left(E_{e}\right)$, and $\bar{K}_{e}\left(E_{e}\right)$ are defined by the expressions in Eq. (B3). The last term in Eq. (F1) is equal to

$$
\begin{aligned}
\left.\frac{d^{5} \lambda_{n}\left(E_{e}, \vec{k}_{e}, \vec{k}_{\bar{\nu}}, \vec{\xi}_{n}, \vec{\xi}_{e}\right)}{d E_{e} d \Omega_{e} d \Omega_{\bar{\nu}}}\right|_{\mathrm{WC}}= & \left(1+3 g_{A}^{2}\right) \frac{\left|G_{V}\right|^{2}}{16 \pi^{5}}\left(E_{0}-E_{e}\right)^{2} \sqrt{E_{e}^{2}-m_{e}^{2}} E_{e} F\left(E_{e}, Z=1\right) \\
& \times\left\{-B_{0} \frac{\pi \alpha}{\beta^{3}} \frac{E_{0}-E_{e}}{m_{N}}\left(\frac{\left(\vec{\xi}_{n} \cdot \vec{k}_{\bar{\nu}}\right)\left(\vec{\xi}_{e} \cdot \vec{k}_{e}\right)\left(\vec{k}_{e} \cdot \vec{k}_{\bar{\nu}}\right)}{E_{e}^{2} E_{\bar{\nu}}^{2}}-\frac{1}{3} \frac{\left(\vec{\xi}_{n} \cdot \vec{k}_{e}\right)\left(\vec{\xi}_{e} \cdot \vec{k}_{e}\right)}{E_{e}^{2}}\right)\right\} .
\end{aligned}
$$

These contributions to the electron-energy and angular distribution of the neutron beta decay vanish after the integration over the directions of the antineutrino 3-momentum $\vec{k}_{\bar{\nu}}$. Because of the contributions of Wilkinson's corrections, caused by the proton recoil in the electron-proton final-state
Coulomb interaction, and the $O\left(\alpha E_{e} / m_{N}\right)$ outer radiative corrections [see Eq. (D10)], the electron-energy and angular distributions in Eqs. (F2)-(F8) are well-defined in the experimental electron-energy region $0.811 \mathrm{MeV} \leq E_{e} \leq$ $1.211 \mathrm{MeV}$ [5].
[1] H. Abele, The neutron. Its properties and basic interactions, Prog. Part. Nucl. Phys. 60, 1 (2008).

[2] J. S. Nico, Neutron beta decay, J. Phys. G 36, 104001 (2009).

[3] S. Paul, The puzzle of neutron lifetime, Nucl. Instrum. Methods Phys. Res., Sect. A 611, 157 (2009).

[4] H. Abele, Precision experiments with cold and ultra-cold neutrons, Hyperfine Interact. 237, 155 (2016).

[5] B. Märkisch, H. Mest, H. Saul, X. Wang, H. Abele, D. Dubbers, M. Klopf, A. Petoukhov, C. Roick, T. Soldner, and D. Werder, Measurement of the Weak Axial-Vector Coupling Constant in the Decay of Free Neutrons Using a Pulsed Cold Neutron Beam, Phys. Rev. Lett. 122, 242501 (2019).

[6] A. Czarnecki, W. J. Marciano, and A. Sirlin, Neutron Lifetime and Axial Coupling Connection, Phys. Rev. Lett. 120, 202002 (2018).

[7] D. Dubbers and B. Märkisch, Precise measurements of the decay of free neutrons, Annu. Rev. Nucl. Part. Sci. 71, 139 (2021).

[8] K. Bodek, L. De Keukeleere, M. Kolodziej, A. Kozela, M. Kuzniak, K. Lojek, M. Perkowski, H. Przybilski, K. Pysz,
D. Rozpedzik, N. Severijns, T. Soldner, A. R. Young, and J. Zejma, BRAND Search for BSM physics at TeV scale by exploring transverse polarization of electrons emitted in neutron decay, EPJ Web Conf. 219, 04001 (2019).

[9] J. F. Gonoghue, E. Golowich, and B. R. Holstein, in Dynamics of the Standard Model, 2nd ed. (Cambridge University Press, Cambridge, 2014), https://doi.org/ 10.1017/CBO9780511803512.

[10] P. A. Zyla et al. (Particle Data Group), Review of particle physics, Prog. Theor. Exp. Phys. 2020, 083C01 (2020).

[11] A. N. Ivanov, M. Pitschmann, and N. I. Troitskaya, Neutron $\beta$-decay as a laboratory for testing the standard model, Phys. Rev. D 88, 073002 (2013) [arXiv:1212.0332].

[12] A. N. Ivanov, R. Höllwieser, N. I. Troitskaya, M. Wellenzohn, and Ya. A. Berdnikov, Precision analysis of electron energy spectrum and angular distribution of neutron beta decay with polarized neutron and electron, Phys. Rev. C 95, 055502 (2017) [arXiv:1705.07330].

[13] A. N. Ivanov, R. Höllwieser, N. I. Troitskaya, M. Wellenzohn, and Ya. A. Berdnikov, Tests of the standard model in neutron beta decay with polarized neutron and electron and 
an unpolarized proton, Phys. Rev. C 98, 035503 (2018) [arXiv:1805.03880].

[14] A. N. Ivanov, R. Höllwieser, N. I. Troitskaya, M. Wellenzohn, and Ya. A. Berdnikov, Test of the Standard Model in neutron beta decay with polarized electrons and unpolarized neutrons and protons, Phys. Rev. D 99, 053004 (2019) [arXiv:1811.04853].

[15] D. H. Wilkinson, Analysis of neutron beta decay, Nucl. Phys. A377, 474 (1982).

[16] A. N. Ivanov, R. Höllwieser, N. I. Troitskaya, M. Wellenzohn, and Ya. A. Berdnikov, Radiative corrections of order $O\left(\alpha E_{e} / m_{N}\right)$ to Sirlin's radiative corrections of order $O(\alpha / \pi)$ to the neutron lifetime, Phys. Rev. D 99, 093006 (2019) [arXiv:1905.01178].

[17] A. N. Ivanov, R. Höllwieser, N. I. Troitskaya, M. Wellenzohn, and Ya. A. Berdnikov, Radiative corrections of order $O\left(\alpha E_{e} / m_{N}\right)$ to Sirlin's radiative corrections of order $O(\alpha / \pi)$, induced by hadronic structure of the neutron, Phys. Rev. D 103, 113007 (2021) [arXiv:2105.06952].

[18] D. H. Wilkinson and B.E.F. Macfield, The numerical evaluation of radiative corrections of order $\alpha$ to allowed nuclear $\beta$-decay, Nucl. Phys. A158, 110 (1970).

[19] A. Sirlin, General properties of the electromagnetic corrections to the beta decay of a physical nucleon, Phys. Rev. 164, 1767 (1967).

[20] A. Sirlin, Current algebra formulation of radiative corrections in gauge theories and the universality of the weak interactions, Rev. Mod. Phys. 50, 573 (1978).

[21] R. T. Shann, Electromagnetic effects in the decay of polarized neutrons, Nuovo Cimento A 5, 591 (1971).

[22] S. Ando, H. W. Fearing, V. Gudkov, K. Kubodera, F. Myhrer, S. Nakamura, and T. Sato, Neutron betadecay in effective field theory, Phys. Lett. B 595, 250 (2004).

[23] V. Gudkov, G. I. Greene, and J. R. Calarco, General classification and analysis of neutron beta-decay experiments, Phys. Rev. C 73, 035501 (2006).

[24] A. N. Ivanov, R. Höllwieser, N. I. Troitskaya, M. Wellenzohn, and Ya. A. Berdnikov, Corrections of order $O\left(E_{e}^{2} / m_{N}^{2}\right)$, caused by weak magnetism and proton recoil, to the neutron lifetime and correlation coefficients of the neutron beta decay, Results Phys. 21, 103806 (2021) [arXiv:2010.14336].

[25] J. D. Jackson, S. B. Treiman, and H. W. Wyld, Jr., Possible tests of time reversal invariance in beta decay, Phys. Rev. 106, 517 (1957).

[26] J. D. Jackson, S. B. Treiman, and H. W. Wyld, Jr., Coulomb corrections in allowed beta transitions, Nucl. Phys. 4, 206 (1957).

[27] J. D. Jackson, S. B. Treiman, and H. W. Wyld, Jr., Note on relativistic coulomb wave functions, Z. Phys. 150, 640 (1958).

[28] M. E. Ebel and G. Feldman, Further remarks on Coulomb corrections in allowed beta transitions, Nucl. Phys. 4, 213 (1957).

[29] A. N. Ivanov, R. Höllwieser, N. I. Troitskaya, M. Wellenzohn, and Ya. A. Berdnikov, On the correlation coefficient $T\left(E_{e}\right)$ of the neutron beta decay, caused by the correlation structure invariant under discrete $\mathrm{P}, \mathrm{C}$ and $\mathrm{T}$ symmetries, Phys. Lett. B 816, 136263 (2021).
[30] A. N. Ivanov, R. Höllwieser, N. I. Troitskaya, M. Wellenzohn, and Ya.A. Berdnikov, On the structure of the correlation coefficients $S\left(E_{e}\right)$ and $U\left(E_{e}\right)$ of the neutron beta decay, Phys. Rev. C 104, 025503 (2021) arXiv: 2102.02021.

[31] C. Itzykson and J.-B. Zuber, in Quantum Field Theory (McGraw-Hill Inc., New York, 1980).

[32] J. M. Blatt and V.F. Weisskopf, Theoretical Nuclear Physics (John Wiley \& Sons, New York, 1952).

[33] A. Antognini et al., Proton structure from the measurement of $2 \mathrm{~S}-2 \mathrm{P}$ transition frequencies of muonic hydrogen, Science 339, 417 (2013).

[34] M. Fierz, Zur Fermischen Theorie des $\beta$-Zerfalls, Z. Phys. 104, 553 (1937).

[35] J. C. Hardy and I. S. Towner, Superallowed $0^{+} \rightarrow 0^{+}$ nuclear beta decays: 2020 critical survey, with implications for $V_{u d}$ and CKM unitarity, Phys. Rev. C 102, 045501 (2020).

[36] M. González-Alonso, O. Naviliat-Cuncic, and N. Severijns, New physics searches in nuclear and neutron beta decay, Prog. Part. Nucl. Phys. 104, 165 (2019).

[37] H. Saul, Ch. Roick, H. Abele, H. Mest, M. Klopf, A. Petukhov, T. Soldner, X. Wang, D. Werder, and B. Märkisch, Limit on the Fierz Interference Term b from a Measurement of the Beta Asymmetry in Neutron Decay, Phys. Rev. Lett. 125, 112501 (2020).

[38] V. Cirigliano, A. Garcia, D. Gazit, O. Naviliat-Cuncic, G. Savard, and A. Young, Precision beta decay as a probe of new physics, arXiv:1907.02164.

[39] X. Sun et al. (UCNA Collaboration), Improved limits on Fierz interference using asymmetry measurements from the ultracold neutron asymmetry (UCNA) experiment, Phys. Rev. C 101, 035503 (2020).

[40] A. N. Ivanov, R. Höllwieser, N. I. Troitskaya, M. Wellenzohn, and Ya.A. Berdnikov, Neutron dark matter decays and correlation coefficients of neutron beta decays, Nucl. Phys. B938, 114 (2019).

[41] A. N. Ivanov, R. Höllwieser, N. I. Troitskaya, M. Wellenzohn, and Ya. A. Berdnikov, Precision analysis of pseudoscalar interactions in neutron beta decays, Nucl. Phys. B951, 114891 (2020) [arXiv:1905.04147].

[42] T. D. Lee and C.N. Yang, Charge conjugation, a new quantum number $G$, and selection rules concerning a nucleon anti-nucleon system, Nuovo Cimento 3, 749 (1956).

[43] S. Weinberg, Charge symmetry of weak interactions, Phys. Rev. 112, 1375 (1958).

[44] S. Gardner and C. Zhang, Sharpening Low-Energy, Standard-Model Tests via Correlation Coefficients in Neutron Beta Decay, Phys. Rev. Lett. 86, 5666 (2001).

[45] S. Gardner and B. Plaster, Framework for maximum likelihood analysis of neutron beta decay observables to resolve the limits of the $V-A$ law, Phys. Rev. C 87, 065504 (2013).

[46] The numerical analysis of the correlation function $\zeta\left(E_{e}\right)$ and correlation coefficients $a\left(E_{e}\right), A\left(E_{e}\right), \ldots, U\left(E_{e}\right)$ we have carried out by Wolfram Mathematica 12. The analytical expressions for the correlation function and correlation coefficients are given in Appendix E programmed in the $\mathrm{nb}$ file, where one may find plotted radiative corrections $O\left(\alpha E_{e} / m_{N}\right)$ in Eqs. (A20) and (D10). This nb file can be 
sent by a request for practical applications of the results, obtained in this work.

[47] FS. M. Bilen'kii, R. M. Ryndin, Ya. A. Smorodinskii, and Ho Tso-Hsiu, On the theory of the neutron beta decay, JETP 37, 1759 (1959) [Sov. Phys. JETP 37, 1241 (1960)].

[48] W. J. Marciano and A. Sirlin, Radiative Corrections to $\beta$ Decay and the Possibility of a Fourth Generation, Phys. Rev. Lett. 56, 22 (1986).

[49] A. Czarnecki, W. J. Marciano, and A. Sirlin, Precision measurements and CKM unitarity, Phys. Rev. D 70, 093006 (2004).

[50] W. J. Marciano and A. Sirlin, Improved Calculation of Electroweak Radiative Corrections and the Value of $V(u d)$, Phys. Rev. Lett. 96, 032002 (2006).

[51] Ch.-Y. Seng, M. Gorchtein, H. H. Patel, and M. J. RamseyMusolf, Reduced Hadronic Uncertainty in the Determination of $V_{u d}$, Phys. Rev. Lett. 121, 241804 (2018).

[52] C.-Y. Seng, M. Gorchtein, and M. J. Ramsey-Musolf, Dispersive evaluation of the inner radiative correction in neutron and nuclear beta decay, Phys. Rev. D 100, 013001 (2019).

[53] A. Czarnecki, W. J. Marciano, and A. Sirlin, Radiative corrections to neutron and nuclear beta decays revisited, Phys. Rev. D 100, 073008 (2019).

[54] L. Hayen, Standard Model $(\alpha)$ renormalization of $g_{A}$ and its impact on new physics searches, Phys. Rev. D 103, 113001 (2021).

[55] L. Hayen, Radiative corrections to nucleon weak charges and beyond Standard Model impact, arXiv:2102.03458.

[56] M. Gorchtein and C.-Y. Seng, Dispersion relation analysis of the radiative corrections to $g_{A}$ in the neutron beta decay, arXiv:2106.09185.

[57] S. M. Berman, Radiative corrections to muon and neutron decay, Phys. Rev. 112, 267 (1958).

[58] T. Kinoshita and A. Sirlin, Radiative corrections to Fermi interactions, Phys. Rev. 113, 1652 (1959).

[59] S. M. Berman and A. Sirlin, Some considerations on the radiative corrections to muon and neutron decay, Ann. Phys. (N.Y.) 20, 20 (1962).

[60] G. Källén, Radiative corrections to beta decay and nucleon form factors, Nucl. Phys. B1, 225 (1967).

[61] E. S. Abers, D. A. Dicus, R. E. Norton, and H. R. Queen, Radiative corrections to the Fermi part of strangenessconserving beta decay, Phys. Rev. 167, 1461 (1968).

[62] A. García and M. Maya, First-order radiative corrections to asymmetry coefficients in neutron decay, Phys. Rev. D 17, 1376 (1978).

[63] Yu. V. Gaponov and R. U. Khafisov, Radiative neutron beta decay and its possible experimental realization, Phys. Lett. B 379, 7 (1996).

[64] V. Bernard, S. Gardner, U.-G. Meißner, and C. Zang, Radiative neutron beta decay in effective field theory, Phys. Lett. B 593, 105 (2004).

[65] F. Glück, Measurable distributions of unpolarized neutron decay, Phys. Rev. D 47, 2840 (1993).

[66] F. Glück, I. Joó, and J. Last, Measurable parameters of neutron decay, Nucl. Phys. A593, 125 (1995).

[67] F. Glück, Monte Carlo type radiative corrections for neutron, muon and hyperon semileptonic decays, Comput. Phys. Commun. 95, 111 (1996).
[68] F. Glück, Order-alpha radiative correction calculations for unoriented allowed nuclear, neutron and pion beta decays, Comput. Phys. Commun. 101, 223 (1997).

[69] F. Glück, Electron spectra and electron-proton asymmetries in polarized neutron decay, Phys. Lett. B 436, 25 (1998).

[70] D. Mund, B. Märkisch, M. Deissenroth, J. Krempel, M. Schumann, H. Abele, A. Petoukhov, and T. Soldner, Determination of the Weak Axial Vector Coupling from a Measurement of the Beta-Asymmetry Parameter A in Neutron Beta Decay, Phys. Rev. Lett. 110, 172502 (2013).

[71] M. P. Mendenhall, R. W. Pattie, Jr., Y. Bagdasarova, D. B. Berguno, L. J. Broussard, R. Carr, S. Currie et al. (UCNA Collaboration), Precision measurement of the neutron beta decay asymmetry, Phys. Rev. C 87, 032501 (2013).

[72] M. A.-P. Brown (UCNA Collaboration) et al., New result for the neutron beta-asymmetry parameter $A_{0}$ from UCNA, Phys. Rev. C 97, 035505 (2018).

[73] A. P. Serebrov, O. M. Zherebtsov, A. N. Murashkin, G. N. Klyushnikov, and A.K. Fomin, On the possibility of measuring the ratio $G_{A} / G_{V}$ by means of polarized ultracold neutrons, Yad. Fiz. 82, 110 (2019) [Phys. At. Nucl. 82, 98 (2019)].

[74] I. A. Kuznetsov, A. P. Serebrov, I. V. Stepanenko, A. V. Alduschenkov, M. S. Lasakov, A. A. Kokin, Yu. A. Mostovoi, B. G. Yerozolimsky, and M. S. Dewey, Measurements of the Antineutrino Spin Asymmetry in Beta Decay of the Neutron and Restrictions on the Mass of a Right-Handed Gauge Boson, Phys. Rev. Lett. 75, 794 (1995).

[75] M. Kreuz, T. Soldner, S. Bäßler, B. Brand, F. Glück, U. Mayer, D. Mund, V. Nesvizhevsky, A. Petoukhov, C. Plonka, J. Reich, C. Vogel, and H. Abele, A measurement of the antineutrino asymmetry B in free neutron decay, Phys. Lett. B 619, 263 (2005).

[76] M. Schumann, T. Soldner, M. Deissenroth, F. Glück, J. Krempel, M. Kreuz, B. Mrkisch, D. Mund, A. Petoukhov, and H. Abele, Measurement of the Neutrino Asymmetry Parameter B in Neutron Decay, Phys. Rev. Lett. 99, 191803 (2007).

[77] G. Darius, W. A. Byron, C. R. DeAngelis et al., Measurement of the Electron-Antineutrino Angular Correlation in Neutron Beta Decay, Phys. Rev. Lett. 119, 042502 (2017).

[78] M. Beck, F. A. Guardia, M. Borg et al., Improved determination of the $\beta-\bar{\nu}_{e}$ angular correlation coefficient a in free neutron decay with the aSPECT spectrometer, Phys. Rev. C 101, 055506 (2020).

[79] M. T. Hassan, W. A. Byron, G. Darius et al., Measurement of the neutron decay electron-antineutrino angular correlation by the aCORN experiment, Phys. Rev. C 103, 045502 (2021).

[80] M. Schumann, M. Kreuz, M. Deissenroth, F. Glück, J. Krempel, B. Märkisch, D. Mund, A. Petoukhov, T. Soldner, and H. Abele, Measurement of the Proton Asymmetry Parameter in Neutron Beta Decay, Phys. Rev. Lett. 100, 151801 (2008).

[81] A. N. Ivanov, R. Höllwieser, N. I. Troitskaya, and M. Wellenzohn, Proton recoil energy and angular distribution of neutron radiative beta decay, Phys. Rev. D 88, 065026 (2013) [arXiv:1306.4448].

[82] K. Mitchell, XXXII. Tables of the functions $-\int_{0}^{z} \frac{\log (1-y)}{y} d y$, with an account for some properties of this and related 
functions, Lond. Edinburgh Dublin Philos. Mag. J. Sci. 40, 351 (1949).

[83] V. Bernard, N. Kaiser, and Ulf-G. Meißner, Chiral dynamics in nucleons and nuclei, Int. J. Mod. Phys. E 04, 193 (1995).

[84] A. Liesenfeld et al., A measurement of the axial form factor of the nucleon by the $p\left(e, e^{\prime} \pi^{+}\right) n$ reaction at $W=1125 \mathrm{MeV}$, Phys. Lett. B 468, 20 (1999).

[85] A. N. Ivanov, R. Höllwieser, N. I. Troitskaya, M. Wellenzohn, and Ya. A. Berdnikov, Precision analysis of pseudoscalar interactions in neutron beta decays, Nucl. Phys. B951, 114891 (2020) [arXiv:1905.04147].

[86] V. M. Shekhter, On the weak-interaction types possible in the scheme of Feynman and Gell-Mann, Sov. Phys. JETP 35, 316 (1959).

[87] A. N. Ivanov, R. Höllwieser, N. I. Troitskaya, M. Wellenzohn, and Ya. A. Berdnikov, Precision theoretical analysis of neutron radiative beta decay, Phys. Rev. D 95, 033007 (2017). 